\title{
Synthesis and Evaluation of the First Fluorescent Antagonists of the Human P2Y 2 Receptor Based on AR-C118925
}

\author{
Sean Conroy, ${ }^{\dagger, \perp}$ Nicholas D. Kindon, ${ }^{\dagger, \perp}$ Jacqueline Glenn, ${ }^{\ddagger, \S}$ Leigh A. Stoddart, ${ }^{\ddagger, \S}$ Richard J. Lewis, ${ }^{\|}$
} Stephen J. Hill, ${ }^{\ddagger}, \S$ Barrie Kellam, ${ }^{\dagger, \S(1)}$ and Michael J. Stocks*, ${ }^{\dagger}$

${ }^{\dagger}$ School of Pharmacy, Centre for Biomolecular Sciences, University Park Nottingham, Nottingham NG7 2RD, U.K.

${ }^{\ddagger}$ Division of Physiology, Pharmacology \& Neuroscience, Medical School, University of Nottingham, Nottingham NG7 2UH, U.K.

${ }^{\S}$ Centre of Membrane Proteins and Receptors, University of Birmingham and University of Nottingham, the Midlands NG7 2UH, U.K.

"Medicinal Chemistry, Respiratory, Inflammation and Autoimmunity, IMED Biotech Unit, AstraZeneca, Mölndal, Gothenburg 431 83, Sweden

Supporting Information

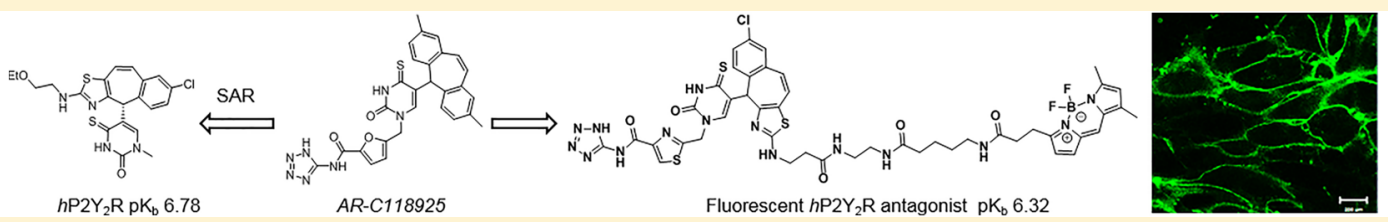

ABSTRACT: The human $\mathrm{P}_{2} \mathrm{Y}_{2}$ receptor $\left(h \mathrm{P}_{2} \mathrm{Y}_{2} \mathrm{R}\right)$ is a G-protein-coupled receptor that shows promise as a therapeutic target for many important conditions, including for antimetastatic cancer and more recently for idiopathic pulmonary fibrosis. As such, there is a need for new $h \mathrm{P}_{2} \mathrm{Y}_{2} \mathrm{R}$ antagonists and molecular probes to study this receptor. Herein, we report the development of a new series of non-nucleotide $h \mathrm{P} 2 \mathrm{Y}_{2} \mathrm{R}$ antagonists, based on the known, non-nucleotide $h \mathrm{P} 2 \mathrm{Y}_{2} \mathrm{R}$ antagonist AR-C118925 (1), leading to the discovery of a series of fluorescent ligands containing different linkers and fluorophores. One of these conjugates, 98, displayed micromolar affinity for $h \mathrm{P}_{2} \mathrm{Y}_{2} \mathrm{R}\left(\mathrm{p} K_{\mathrm{d}}=6.32 \pm 0.10, n=17\right)$ in a bioluminescence-energy-transfer (BRET) assay. Confocal microscopy with this ligand revealed displaceable membrane labeling of astrocytoma cells expressing untagged $h \mathrm{P} 2 \mathrm{Y}_{2} \mathrm{R}$. These properties make $\mathbf{9 8}$ one of the first tools for studying $h \mathrm{P}_{2} \mathrm{Y}_{2} \mathrm{R}$ distribution and organization.

\section{INTRODUCTION}

P2Y receptors (P2YRs) are G-protein-coupled receptors (GPCRs) that are activated by extracellular nucleotides. The P2Y family is composed of eight members, encoded by distinct genes, that can be subdivided into two groups on the basis of their primary signaling through specific $G$ proteins ${ }^{1}$ and sequence homology. The first subgroup includes the $\mathrm{P} 2 \mathrm{Y}_{1,2,4,6,11}$ receptors, which primarily signal though $G_{q}$, whereas the second subgroup, signaling through $G_{i}$, encompasses the $P 2 Y_{12,13,14}$ receptor subtypes. ${ }^{2}$ Notably, the $\mathrm{P}_{2} \mathrm{Y}_{2}$ receptor $\left(\mathrm{P} 2 \mathrm{Y}_{2} \mathrm{R}\right)$ is activated by the endogenous agonists uridine- 5 -triphosphate (UTP, $h \mathrm{P} 2 \mathrm{Y}_{2} \mathrm{EC}_{50}=140 \mathrm{nM}$ ) and adenosine-5'-triphosphate (ATP, $\left.h \mathrm{P} 2 \mathrm{Y}_{2} \mathrm{EC}_{50}=230 \mathrm{nM}\right) .^{3}$ As $\mathrm{P}_{2} \mathrm{Y}_{2} \mathrm{R}$ is predominately $\mathrm{G}_{\mathrm{q}}$ coupled, receptor activation leads to the stimulation of phospholipase $\mathrm{C}, \mathrm{IP}_{3}$ release, and the elevation of the intracellular $\mathrm{Ca}^{2+}$ concentration, as well as the initiation of protein kinase $\mathrm{C}$ and the activation of the mitogen-activated proteinkinase cascade.

Defining the clinical role for $\mathrm{P}_{2} \mathrm{Y}_{2} \mathrm{R}$ antagonism has been hampered by the lack of high-affinity and druglike receptor antagonists. ${ }^{4}$ However, it has been reported that ATP released from tumor-cell-activated platelets induces the opening of the endothelial barrier, leading to the migration of tumor cells and hence cancer proliferation. More importantly, $\mathrm{P}_{2} \mathrm{Y}_{2} \mathrm{R}$ was identified as the primary mediator of this effect; a strong reduction of tumor cell metastasis was observed in $\mathrm{P} 2 \mathrm{Y}_{2} \mathrm{R}$-deficient mice, revealing a therapeutic potential of $\mathrm{P}_{2} \mathrm{Y}_{2} \mathrm{R}$ antagonists as antimetastatic agents. ${ }^{5,6}$ Recently, it has been reported that both inflammation and fibrosis were reduced in $\mathrm{P} 2 \mathrm{Y}_{2} \mathrm{R}$-deficient mice compared with those in wild-type animals. In addition, mechanistic studies have demonstrated that the recruitment of neutrophils into the lungs, the proliferation and migration of lung fibroblasts, and IL-6 production are all key $\mathrm{P} 2 \mathrm{Y}_{2} \mathrm{R}$-mediated processes. These studies clearly demonstrate the involvement of $\mathrm{P}_{2} \mathrm{Y}_{2} \mathrm{R}$ subtypes in the pathogenesis of fibrotic lung diseases in humans and mice and support the development of selective $\mathrm{P}_{2} \mathrm{Y}_{2} \mathrm{R}$ antagonists for the treatment of idiopathic pulmonary fibrosis (IPF). ${ }^{7}$ To date, the only reported high-affinity $\mathrm{P}_{2} \mathrm{Y}_{2} \mathrm{R}$ antagonists were those developed by scientists from AstraZeneca resulting in the non-nucleotide $\mathrm{P}_{2} \mathrm{Y}_{2} \mathrm{R}$ antagonist AR-C118925 (1). ${ }^{8,9}$

Several in vivo and ex vivo studies using 1 have been reported that further validate the therapeutic benefits of $P 2 Y_{2} R$ antagonists. Importantly, it was shown that $\mathbf{1}$, which was reported to be inactive at $10 \mu \mathrm{M}$ against a panel of 37 other

Received: January 27, 2018

Published: March 20, 2018 


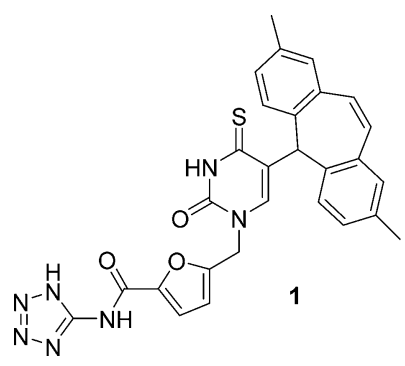

receptors, was able to concentration-dependently antagonize ATP $\gamma$ S-induced mucin secretion in an ex vivo model of human bronchial epithelial cells. ${ }^{10}$ In addition, Müller et al. recently demonstrated that $\mathbf{1}$ was a selective, high-affinity, reversible antagonist of $\mathrm{P}_{2} \mathrm{Y}_{2} \mathrm{R}^{11}$

We were drawn to the exciting possibility of using $\mathbf{1}$ as a chemical template to design new $\mathrm{P} 2 \mathrm{Y}_{2} \mathrm{R}$ antagonists ${ }^{12}$ and synthesize fluorescently labeled chemical tools to further probe $\mathrm{P}_{2} \mathrm{Y}_{2} \mathrm{R}$ function. ${ }^{13}$ Using fluorescence as a means to study GPCRs allows scientists access to a large range of pharmacological techniques that can capture dynamic processes in living cells. ${ }^{14}$ In particular, fluorescently labeled receptor antagonists have been developed to target GPCRs, allowing the visualization of GPCR function at the cellular level. ${ }^{15-17}$ In addition, fluorescent ligands can be used in resonance-energy-transfer (RET) techniques, in particular those that utilize nanoluciferase (NLuc), to quantify ligand-receptor interactions and determine the affinities of unlabeled ligands. ${ }^{18}$ This offers advantages for receptors such as $\mathrm{P}_{2} \mathrm{Y}_{2} \mathrm{R}$ for which there are currently no commercially available radio ligands. In addition, as the reported antagonists for $\mathrm{P} 2 \mathrm{Y}_{2} \mathrm{R}$ have mid to high affinities, it is proposed that the fluorescent ligands designed from these might also have affinities in this range. This may prove problematic for techniques which directly monitor fluorescent-ligand binding, but NanoBRET has been shown to display low, nonspecific binding at high fluorescent-ligand concentrations. ${ }^{18,19}$

\section{RESULTS AND DISCUSSION}

Synthesis and Evaluation of the $h \mathrm{P} 2 \mathrm{Y}_{2} \mathrm{R}$ Antagonists. The medicinal-chemistry strategy involved an initial exploration of the structure-activity relationship (SAR) around $\mathbf{1}$ in order to enable the design of structural analogues with improved predicted physicochemical properties and to guide our design strategy by highlighting suitable linking sites to attach the fluorophore groups (Figure 1 and Table 1).

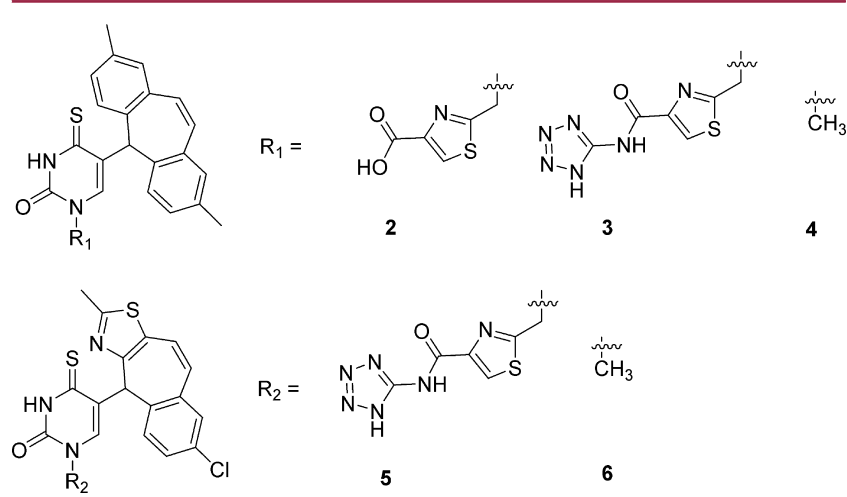

Figure 1. SARs for the $\mathrm{P}_{2} \mathrm{Y}_{2} \mathrm{R}$ antagonists showing the change from the furan in $\mathbf{1}$ to thiazole (reduced lipophilicity) and the change from 2,8-dimethyl-5H-dibenzo[a,d][7]annulene to the 7-chloro-4H-benzo$[5,6]$ cyclohepta $[1,2-d]$ thiazole tricyclic ring system.
Table 1. Estimated Affinity Values for $\mathrm{P}_{2} \mathrm{Y}_{2} \mathrm{R}$ Antagonists 1-6 Obtained from the Calcium-Mobilization Assay

$\begin{array}{cccc}\text { example } & h \mathrm{P} 2 \mathrm{Y}_{2} \mathrm{pK}_{\mathrm{b}}{ }^{a} & \text { example } & h \mathrm{P} 2 \mathrm{Y}_{2} \mathrm{pK}_{\mathrm{b}}{ }^{a} \\ \mathbf{1} & 7.51 \pm 0.09(12) & 4 & \mathrm{IA}^{b} \\ \mathbf{2}^{c} & 6.43 \pm 0.08(3) & \mathbf{5} & 6.48 \pm 0.10(3) \\ \mathbf{3} & 7.11 \pm 0.14(7) & \mathbf{6} & 5.99 \pm 0.03(7)\end{array}$

${ }^{a_{T}}$ The estimated affinity value for each antagonist $\left(\mathrm{p} K_{\mathrm{b}}\right)$ was calculated using the Gaddum equation from the shift of the UTP $\gamma \mathrm{S}$ concentrationresponse curve brought about by the addition of a single concentration of the antagonist. The data shown are the means \pm SEM, and the numbers of separate experiments are given in parentheses. ${ }^{b} \mathrm{IA}=$ inactive; i.e., less than a $50 \%$ inhibition of the response to $0.1 \mu \mathrm{M} \mathrm{UTP} \gamma \mathrm{S}$ in the presence of a $10 \mu \mathrm{M}$ concentration of the compound. UTP $\gamma \mathrm{S} \mathrm{EC}_{50}=$ $7.9 \pm 1.3 \mathrm{nM}(n=25){ }^{c}$ The literature value is $\mathrm{pA}_{2}=5.7 .^{8}$

1 has previously been shown to have a high lipophilicity $(\mathrm{cLogP}=4.2)^{20}$ and poor physicochemical properties for oral delivery. ${ }^{8}$ We therefore synthesized the thiazole analogue, $3(\mathrm{cog} \mathrm{P}=3.8)$, with little loss of affinity for $\mathrm{P}_{2} \mathrm{Y}_{2} \mathrm{R}$. Replacement of the 2,8-dimethyl-5H-dibenzo[a,d][7]annulene with a 7-chloro- $4 H$-benzo $[5,6]$ cyclohepta $[1,2-d]$ thiazole tricyclic ring gave $5\left(\mathrm{p} K_{\mathrm{b}}=6.5, \operatorname{cog} \mathrm{P}=3.4\right) .{ }^{21}$ In addition, a nonparallel SAR with $\mathrm{P}_{2} \mathrm{Y}_{2} \mathrm{R}$ affinity was observed in the replacement of the $N-1$ thiouracil substituent with a methyl group, which gave compound $\mathbf{6}$, whereas the corresponding analogue, 4 , proved inactive. In order to explore this intriguing finding and study this SAR, we synthesized a range of analogues of 6 (Table 2).

Table 2. Calcium-Mobilization Activities for the $\mathrm{P}_{2} \mathrm{Y}_{2} \mathrm{R}$ Antagonists 6-19

\begin{tabular}{|c|c|c|}
\hline example & $\mathrm{R}_{3}$ & $h \mathrm{P} 2 \mathrm{Y}_{2} \mathrm{p}_{\mathrm{b}}{ }^{a}$ \\
\hline 7 & $\mathrm{NH}_{2}$ & $6.56 \pm 0.14$ \\
\hline 8 & $\mathrm{PhNH}$ & $\mathrm{IA}^{b}(3)$ \\
\hline 9 & $\mathrm{PhCH}_{2} \mathrm{NH}$ & IA (3) \\
\hline 10 & $\mathrm{PhCH}_{2} \mathrm{CH}_{2} \mathrm{NH}$ & IA (3) \\
\hline 11 & 1-methyl piperazin-4-yl & IA (3) \\
\hline 12 & morpholinyl & IA (3) \\
\hline 13 & piperidinyl & IA (3) \\
\hline 14 & 2-methoxyethan-1-aminyl & $6.60 \pm 0.21$ \\
\hline 15 & 2-methoxypropan-1-aminyl & $6.56 \pm 0.09$ \\
\hline 16 & 2-ethoxyethan-1-aminyl & $6.73 \pm 0.05$ \\
\hline 17 & 2-isopropoxyethan-1-aminyl & $6.49 \pm 0.09$ \\
\hline 18 & 2-phenoxyethan-1-aminyl & IA (3) \\
\hline 19 & phenyl & IA (3) \\
\hline
\end{tabular}

${ }^{a}$ The estimated affinity value for each antagonist $\left(\mathrm{p} K_{\mathrm{b}}\right)$ was calculated using the Gaddum equation from the shift of the UTP $\gamma S$ concentrationresponse curve brought about by the addition of a single concentration of the antagonist. The data shown are the means \pm SEM, and the numbers of separate experiments are given in parentheses. ${ }^{b} \mathrm{IA}=$ inactive; i.e., less than a $50 \%$ inhibition of the response to $0.1 \mu \mathrm{M} \mathrm{UTP} \gamma \mathrm{S}$ in the presence of a $10 \mu \mathrm{M}$ concentration of the compound $(n=3)$.

From the SAR study of 6 , it was shown that replacing the thiazole 2-methyl substituent with an amino group increased $h \mathrm{P} 2 \mathrm{Y}_{2} \mathrm{R}$ affinity (compare 6 with 7 ), whereas a sterically 
demanding substituent, such as that in 19 , resulted in a complete loss of affinity for $h \mathrm{P}_{2} \mathrm{Y}_{2} \mathrm{R}$. We therefore explored the substitution of the amino group and showed that both compounds with sterically demanding amino groups $(8,9$, and 10) and compounds with cyclic tertiary amines $(11,12$, and 13) were inactive. However, the linear, less sterically demanding alkyl amino groups, such as those in 14, 15, 16, and 17, increased $h \mathrm{P} 2 \mathrm{Y}_{2} \mathrm{R}$ affinity, although the bulkier 2-phenoxyethan-1-amino substituent in 18 resulted in the compound being inactive.

Thus far, all of the compounds synthesized were tested as racemic mixtures. To try and determine whether the activity resided in one enantiomer, the resolution of $\mathbf{1 4}$ and $\mathbf{1 6}$ was achieved through semipreparative chiral HPLC, and the biology of each of the resolved enantiomers was independently assessed (Table 3).

Table 3. Calcium-Mobilization Activities for the Resolved Enantiomers of 14 and 16

$\begin{array}{cccc}\text { example } & \begin{array}{c}\text { racemic } \\ \text { compound }\end{array} & \begin{array}{c}\text { enantiomeric excess } \\ (\mathrm{ee})^{a}\end{array} & h \mathrm{P} 2 \mathrm{Y}_{2} \mathrm{p} K_{\mathrm{b}}{ }^{b} \\ 20 & 14 & 99 \% & 6.63 \pm 0.11(6) \\ 21 & & 78 \% & 5.82 \pm 0.05(3) \\ 22 & 16 & 95 \% & \mathrm{IA}^{c}(3) \\ 23 & & 96 \% & 6.78 \pm 0.05(6)\end{array}$

${ }^{a}$ The compounds were separated using Phenomenex's Lux $5 \mu \mathrm{m}$ amylose-2 stationary phase. ${ }^{b}$ The estimated affinity value for each antagonist $\left(\mathrm{p} K_{\mathrm{b}}\right)$ was calculated using the Gaddum equation from the shift of the UTP $\gamma \mathrm{S}$ concentration-response curve brought about by the addition of a single concentration of the antagonist. The data shown are the means \pm SEM, and the numbers of separate experiments are given in parentheses. ${ }^{c} \mathrm{IA}=$ inactive; i.e., less than a $50 \%$ inhibition of the response to $0.1 \mu \mathrm{M} \mathrm{UTP} \gamma \mathrm{S}$ in the presence of a $10 \mu \mathrm{M}$ concentration of the compound $(n=3)$.

From these results, it is possible to see that the $h \mathrm{P} 2 \mathrm{Y}_{2} \mathrm{R}$ antagonist affinities observed for the racemic compounds 14 and 16 reside largely in enantiomers 20 and 23, respectively. Although some antagonist activity is observed for 21, this may be attributed to residual active enantiomer 20, which constitutes $11 \%$ of the sample. Unfortunately, the resolved enantiomers proved to be amorphous powders, and so singlecrystal X-ray determination of their absolute chiralities could not be used for structural determination. However, vibrational circular dichroism was used for $\mathbf{2 2}$ and 23, from which spectra were acquired for both samples and fitted to the calculated spectra. $^{22-24}$ The results (Supporting Information) showed that there was a good match between the spectrum of 22 and the calculated spectrum for the $(S)$-enantiomer; therefore, 23 was assigned as the active $(R)$-enantiomer (Figure 2 ).

In an attempt to increase affinity within the new series of compounds, we the incorporated key structural features of 14 and 5 to generate compound 24 .

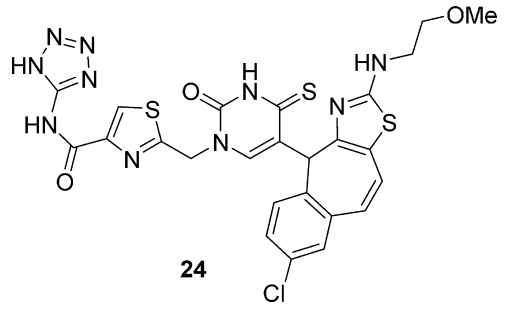

However, 24 did not demonstrate the expected increase in affinity from the combination of features from 14 and 5 and instead showed a level of $h \mathrm{P} 2 \mathrm{Y}_{2} \mathrm{R}$ affinity $\left(\mathrm{p} K_{\mathrm{d}}=7.02 \pm 0.05\right.$, $n=4)$ similar to those of $\mathbf{1}$ and $\mathbf{3}$, demonstrating that the SARs within the series of compounds were nonadditive. ${ }^{25}$

The synthesis of compounds $\mathbf{2 - 4}$ is illustrated in Scheme 1. The alkylation of 5-(2,8-dimethyl-5H-dibenzo[ $a, d][7]$ annulen5-yl)pyrimidine-2,4 $(1 H, 3 H)$-dione ${ }^{8}$ with ethyl 2-(bromomethyl)thiazole-4-carboxylate followed by a treatment with Lawesson's reagent and saponification gave $2,{ }^{8}$ which was reacted with 2-amino tetrazole via benzotriazol-1-yl-oxytripyrrolidinophosphonium hexafluorophosphate activation to afford 3. In a similar manner, alkylation with methyl iodide followed by a conversion to the thiouracil gave 4 in a good overall yield.

Scheme 2 shows the synthetic route to compounds 5 and $\mathbf{6}$. The first step involved performing a Heck reaction coupling 3-chloroiodobeneze, 25, to allyl alcohol, and this successfully isomerized in situ yielding the desired aldehyde, 26. This compound was reacted with ethyl dichloroacetate in a Darzens condensation $^{25}$ to generate an $\alpha$-chloro epoxide, which was reacted directly with thioacetamide to afford the desired 2-methylthiazole, 27, with moderate yields achieved over two steps. Freshly prepared sodium ethoxide, generated from sodium metal in dried ethanol, was found to be the optimal base for the Darzens condensation. Saponification gave the carboxylic acid, $\mathbf{2 8}$, and treatment with oxalyl chloride generated the acyl chloride, which was immediately cyclized to give the tricyclic ketone, 29, as a single regioisomer. Lithiation of the di-tert-butyl etherprotected uracil, $30,{ }^{25}$ was readily achieved with $n$-butyllithium, and this compound underwent a 1,2-addition to the ketone, 29, to give the tertiary alcohol, 32 . Concomitant deprotection and dehydration resulted in uracil, 33 , via heating in trifluoroacetic acid. Although the yield for this reaction was poor, other acidic conditions were ineffective. Alkylation at the N1-position of the uracil was achieved in a one-pot process of silylation with N,O-bis(trimethylsilyl)trifluoroacetamide (BSTFA), alkylation with iodomethane, and subsequent desilylation, which give 34 . Finally, a reaction with Lawesson's reagent gave 6. From the uracil intermediate, 33, alkylation with ethyl 2-(bromomethyl) thiazole-4-carboxylate $^{26}$ gave 35 , which was subsequently reacted with Lawesson's reagent to give 36. Hydrolysis was

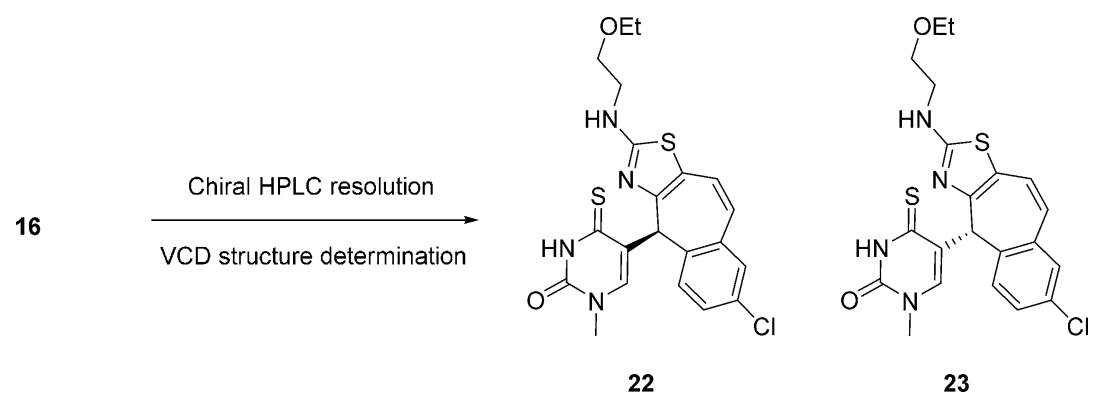

Figure 2. Chiral resolution of $\mathbf{1 6}$ and structural assignment made by vibrational circular dichroism. 
Scheme 1. Synthesis of Compounds $2-4^{a}$
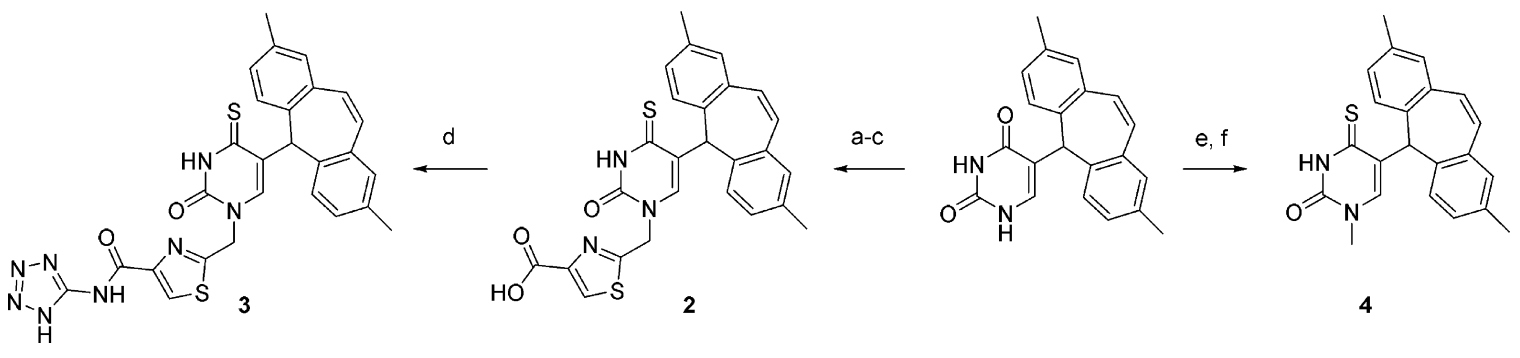

${ }^{a}$ Reagents and conditions: (a) (i) N,O-Bis(trimethylsilyl)trifluoroacetamide, DCM, reflux, $18 \mathrm{~h}$; (ii) ethyl 2-(bromomethyl)thiazole-4-carboxylate, $50{ }^{\circ} \mathrm{C}, 24 \mathrm{~h}(55 \%)$. (b) Lawesson's reagent, 1, 4-dioxane, $100{ }^{\circ} \mathrm{C}, 18 \mathrm{~h}(85 \%)$. (c) $\mathrm{NaOH}$, methanol/ $\mathrm{H}_{2} \mathrm{O}$, reflux, $1 \mathrm{~h}(91 \%)$. (d) 5-Aminotetrazole, benzotriazol-1-yl-oxytripyrrolidinophosphonium hexafluorophosphate, DMF, DIPEA, rt, $1 \mathrm{~h}$ (49\%). (e) (i) N,O-Bis(trimethylsilyl)trifluoroacetamide, DCM, reflux, $18 \mathrm{~h}$; (ii) iodomethane, $50{ }^{\circ} \mathrm{C}, 24 \mathrm{~h}$ (61\%). (f) Lawesson's reagent, 1,4-dioxane, reflux, $18 \mathrm{~h}(40 \%)$.

followed by a reaction of the resulting carboxylic acid with 5-aminotetrazole and benzotriazol-1-yl-oxytripyrrolidinophosphonium hexafluorophosphate to give $\mathbf{5}$.

In a similar sequence to Scheme 2, aldehyde $\mathbf{2 6}$ was reacted with ethyl dichloroacetate, and the resulting crude $\alpha$-chloro epoxide reacted with thiourea to afford the 2-aminothiazole, 37, which was converted to the 2-chlorothiazole, 38 (Scheme 3). The ethyl ester was hydrolyzed to afford the carboxylic acid, 39, which was converted to the acid chloride and cyclized to the tricyclic ketone, 40. Lithiation of the tert-butyl ether-protected uracil, 31, and a reaction with $\mathbf{4 0}$ gave the tertiary alcohol, $\mathbf{4 1}$. After screening a range of milder acidic conditions, heating to $140{ }^{\circ} \mathrm{C}$ with microwave irradiation in acetic acid/1,4-dioxane (1:1) for $10 \mathrm{~min}$ was found to be optimal for the formation of the desired uracil intermediate, 42, which was subsequently methylated to give $\mathbf{4 3}$. The chlorine atom in compound $\mathbf{4 3}$ was readily displaced through a nucleophilic aromatic substitution with a range of primary and secondary amines upon heating under basic conditions. These conditions were unsuccessful when aniline was used, and in this instance, heating in the microwave with hydrochloric acid (catalytic) proved successful in giving 46. Microwave-based conditions were employed for the substitution with ammonia in the synthesis of 45. A Suzuki reaction with phenylboronic acid gave 44. Through the use of Lawesson's reagent, it was then possible to convert these uracil derivatives (44-54) to the respective 4-thiouracil derivatives (6-19). Through a route analogous to the synthesis of compound 7 , it was possible to generate the desired tetrazole analogue, 24 . Uracil intermediate $\mathbf{4 2}$ was alkylated at the N1-position with ethyl 2-(bromomethyl)thiazole-4-carboxylate to give 57. This compound was reacted with 2-methoxyethylamine to afford 58, which was subsequently reacted with Lawesson's reagent to give the 4-thiouracil, 59. Hydrolysis followed by benzotriazol-1yl-oxytripyrrolidinophosphonium hexafluorophosphate activation and a reaction with 5-aminotetrazole afforded 24 .

Synthesis of the $h P 2 Y_{2} R$ Fluorescent Ligands. With a view to developing a series of fluorescent conjugates suitable for both a bioluminescence-resonance-energy-transfer (BRET) ligand-binding assay ${ }^{18,27-29}$ and imaging via confocal microscopy, we embarked on a strategy to synthesize BODIPY conjugates, specifically with the dyes BODIPY A $(628 \mathrm{~nm}$ absorption max, $642 \mathrm{~nm}$ emission max) or BODIPY B (503 nm absorption max, $509 \mathrm{~nm}$ emission max) as this would allow us the opportunity of ligand choice in future imaging work.

Two positions on the $\mathrm{P} 2 \mathrm{Y}_{2} \mathrm{R}$-antagonist core structure were considered for the attachment of the linker and fluorophore (Figure 3). In order to simplify the synthetic chemistry and increase the SARs within the series, we examined the replacement of the furan ring of $\mathbf{1}$ and the thiazole ring of $\mathbf{2}$ with 1,3,5trisubstituted phenyl rings. This would allow the attachment of the acyl tetrazole group in addition to providing a second free carboxylic acid group to which the fluorescent conjugates could be attached. Fortunately, the 3,5-dicarboxylic acid on 60 $\left(h \mathrm{P} 2 \mathrm{Y}_{2} \mathrm{R} \mathrm{p} K_{\mathrm{d}}=6.53 \pm 0.04, n=7\right)$ was well tolerated with no loss of affinity for $\mathrm{P}_{2} \mathrm{Y}_{2} \mathrm{R}$ compared with the affinity of compound 2. Therefore, the first series of compounds had the linker fluorophore attached via the phenyl ring of $\mathbf{6 0}$.

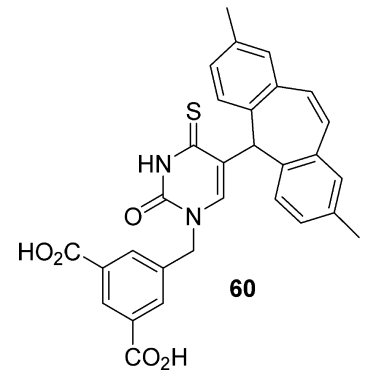

Having established that alkoxyalkyl amines are tolerated in terms of activity in the 2-position of the thiazole in the $4 H$-benzo[5,6]cyclohepta[1,2-d]thiazol-4-yl) tricyclic rings of compounds of the type shown in Figure 2, this position was chosen as the second point of attachment of the linker fluorophore. Finally, a third generation of fluorescent ligands were explored that contained the optimal second-generation fluorescent ligand with an incorporated acyl tetrazole functional group.

First-Generation $h P 2 Y_{2} R$ Fluorescent Ligands. The general synthetic route for the first-generation $\mathrm{P}_{2} \mathrm{Y}_{2} \mathrm{R}$ fluorescent antagonists is shown in Scheme 4.

Alkylation of 5-(2,8-dimethyl-5H-dibenzo[ $a, d][7]$ annulen-5yl)pyrimidine-2,4(1H,3H)-dione ${ }^{8}$ with dimethyl 5-(bromomethyl)isophthalate followed by a treatment with Lawesson's reagent and the selective hydrolysis of one of the methyl esters gave 61. This carboxylic acid (61) was activated using benzotriazol-1-yl-oxytripyrrolidinophosphonium hexafluorophosphate and coupled to form the appropriate amides (62 and 63). The second esters of $\mathbf{6 2}$ and $\mathbf{6 3}$ were then hydrolyzed and converted to amidotetrazoles (64 and 65). Finally, the Boc protecting group was removed, and the resulting amine was coupled to the fluorophore with the appropriate commercially available BODIPY succinimidyl ester to give a small library of four fluorescent conjugates (66-69).

To determine whether any of these conjugates had affinities for $\mathrm{P}_{2} \mathrm{Y}_{2} \mathrm{R}$ and consequently if they could be used in 
Scheme 2. Synthesis of Compounds 5 and $6^{a}$

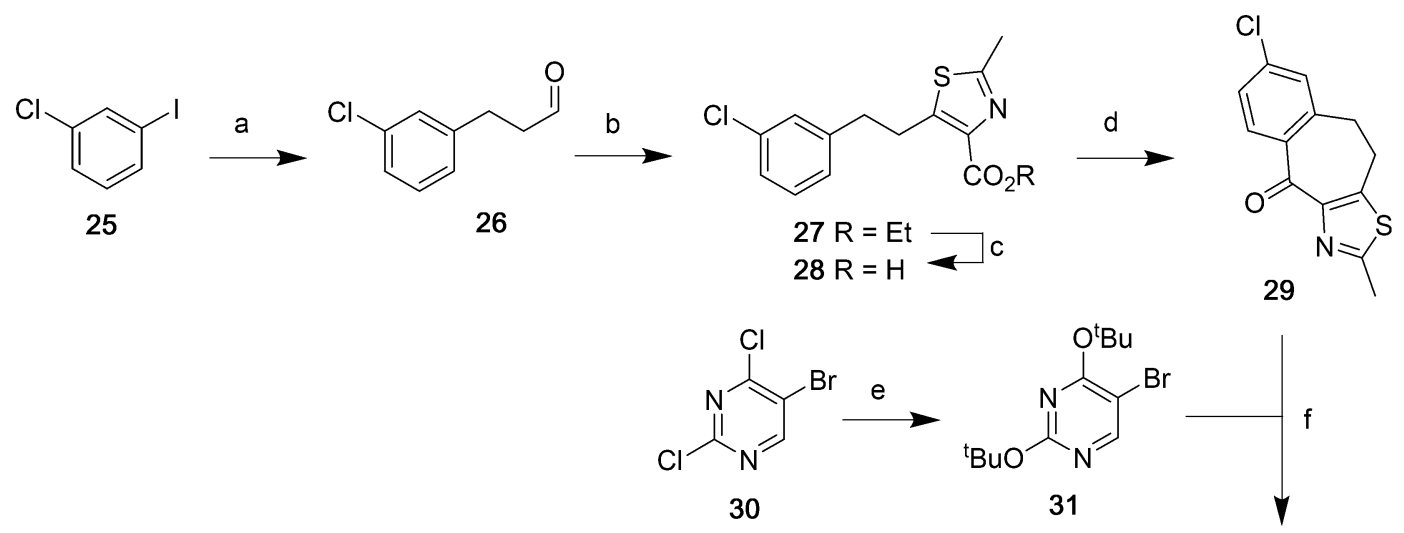<smiles>Cc1nc2c(s1)C=Cc1cc(Cl)ccc1C2c1cn(C)c(=O)[nH]c1=O</smiles><smiles>Cc1nc2c(s1)C=Cc1cc(Cl)ccc1C2c1c[nH]c(=O)[nH]c1=O</smiles>
33<smiles>CCCCOc1ncc(C2(O)c3ccc(Cl)cc3CCc3sc(C)nc32)c(OCCC)n1</smiles>

j<smiles>[Y6]CCc1ccc2c(c1)C=Cc1sc(C)nc1C2c1cn(Cc2nc(C(=O)OCC)cs2)c(=O)[nH]c1=O</smiles>

${ }^{a}$ Reagents and conditions: (a) Allyl alcohol, tetrabutylammonium chloride, DMF, $3 \mathrm{~mol} \%$ palladium(II)acetate, $\mathrm{NaHCO}_{3}, 50{ }^{\circ} \mathrm{C}, 18 \mathrm{~h}(92 \%)$. (b) (i) Ethyl dichloroacetate, sodium ethoxide, diethyl ether, $30 \mathrm{~min}, 0-40{ }^{\circ} \mathrm{C}$; (ii) thioacetamide, ethanol, reflux, $3 \mathrm{~h}$ ( $30 \%$ over two steps). (c) $\mathrm{NaOH}, \mathrm{H}_{2} \mathrm{O} / \mathrm{THF}(1: 1)$, rt, $18 \mathrm{~h}$ (99\%). (d) (i) Oxalyl chloride, catalytic DMF, DCM, rt, 3 h; (ii) aluminum(III)chloride, DCM, rt, 18 h (39\% over two steps). (e) Sodium tert-butoxide, THF, $0{ }^{\circ} \mathrm{C}$ to rt, $18 \mathrm{~h}$ (61\%). (f) (i) $n$-Butyllithium, THF, $-78{ }^{\circ} \mathrm{C}, 30 \mathrm{~min}$; (ii) $29,-78{ }^{\circ} \mathrm{C}$ to rt, $1 \mathrm{~h}$ (65\%). (g) Trifluoroacetic acid, reflux, $72 \mathrm{~h}(36 \%)$. (h) (i) N,O-Bis(trimethylsilyl)trifluoroacetamide, DCM, reflux, $18 \mathrm{~h}$; (ii) iodomethane, $50{ }^{\circ} \mathrm{C}, 24 \mathrm{~h}$ (61\%). (i) Lawesson's reagent, 1,4-dioxane, reflux, $18 \mathrm{~h}$ (40\%). (j) (i) N,O-Bis(trimethylsilyl)trifluoroacetamide, DCM, reflux, $18 \mathrm{~h}$; (ii) ethyl 2-(bromomethyl)thiazole-4-carboxylate, $50{ }^{\circ} \mathrm{C}, 24 \mathrm{~h}$ (57\%). (k) Lawesson's reagent, 1,4-dioxane, $100{ }^{\circ} \mathrm{C}, 18 \mathrm{~h}(89 \%)$. (l) (i) NaOH, methanol/ $\mathrm{H}_{2} \mathrm{O}$, reflux, $1 \mathrm{~h}$ (91\%); (ii) 5-aminotetrazole, benzotriazol-1-yl-oxytripyrrolidinophosphonium hexafluorophosphate, DMF, DIPEA, rt, 1 h (30\%).

NanoBRET binding assays, a $1321 \mathrm{~N} 1$ astrocytoma cell line expressing recombinant $\mathrm{P}_{2} \mathrm{Y}_{2} \mathrm{R}$ tagged on its $\mathrm{N}$-terminus with NLuc (NLuc-P2 ${ }_{2} \mathrm{R}$ ) was generated. The NLuc-tagged $\mathrm{P}_{2} \mathrm{Y}_{2}$ receptors exhibited normal calcium signals $\left(\mathrm{UTP} \gamma \mathrm{S} \mathrm{EC}_{50}=91 \pm\right.$ $12 \mathrm{nM}, n=3$ ). These NLuc-P2Y ${ }_{2}$ cells were treated with increasing concentrations of 66-69 and then treated with the NLuc substrate, furimazine, before the resulting BRET signal was monitored. All four compounds showed moderate to low affinities for the NLuc-P2 ${ }_{2} \mathrm{R}$ (Table 4), with conjugates 68 and 69 having higher affinities. However, this did illustrate the power of using NanoBRET to monitor ligand binding to low-affinity receptors.
Second-Generation $h \mathrm{P} 2 \mathrm{Y}_{2} \mathrm{R}$ Fluorescent Ligands. The general synthetic route for the second-generation $P 2 \mathrm{Y}_{2} \mathrm{R}$ fluorescent antagonists, in which the linker fluorophores are attached to the thiazole rings, is illustrated in Scheme 5.

Nucleophilic displacement of the 2-chloro substituent of $\mathbf{4 3}$ by either tert-butyl 3-aminopropanoate or tert-butyl 6-aminohexanoate gave the corresponding tert-butyl esters (70 and 71). Treatment with Lawesson's reagent in 1,4-dioxane at $100{ }^{\circ} \mathrm{C}$ resulted in the formation of the 4-thiouracils (72 and 73). The carboxylic acids were generated from the tert-butyl esters via TFA-mediated hydrolysis and subsequently coupled with tert-butyl (2-aminoethyl)carbamate or tert-butyl 


\section{Scheme 3. Synthesis of Compound $24^{a}$}<smiles>[R]OCc1nc(Cl)sc1CCc1cccc(Cl)c1</smiles><smiles></smiles><smiles>Cc1nc2c(s1)C=Cc1cc(Cl)ccc1C2c1cn(C)c(=O)[nH]c1=O</smiles><smiles>O=c1[nH]cc(C2c3ccc(Cl)cc3C=Cc3sc(Cl)nc32)c(=O)[nH]1</smiles><smiles>CC(C)(C)C</smiles><smiles>CC(C)(C)Oc1ncc2c(n1)OCCCCO2</smiles><smiles>[R]N([R2])c1nc2c(s1)C=Cc1cc(Cl)ccc1C2C1C=C(C)C(=S)NC1=O</smiles>

7-18<smiles>[R2]Nc1nc2c(s1)C=Cc1cc(Cl)ccc1C2c1cn(C)c(=O)[nH]c1=O</smiles>

45-56<smiles>CCOC(=O)c1csc(Cn2cc(C3c4ccc(Cl)cc4C=Cc4sc(Cl)nc43)c(=O)[nH]c2=O)n1</smiles>

57

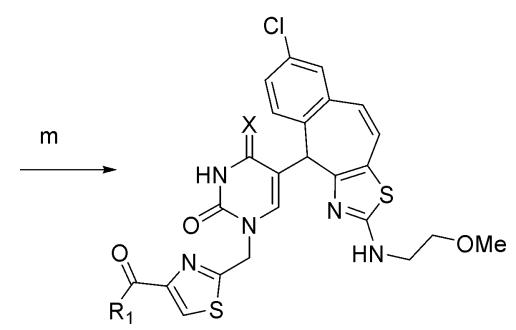

$58 \mathrm{R}_{1}=\mathrm{OEt}, \mathrm{X}=\mathrm{O}$ $59 \mathrm{R}_{1}=$ OEt $\mathrm{X}=\mathrm{S} \rightleftarrows \mathrm{n}$

○

$24 R_{1}=2$-aminotetrazole, $X=S$

${ }^{a}$ Reagents and conditions: (a) (i) Ethyl dichloroacetate, sodium ethoxide, diethyl ether, $30 \mathrm{~min}, 0-40{ }^{\circ} \mathrm{C}$; (ii) thiourea, ethanol, reflux, $2.5 \mathrm{~h}(46 \%$ over two steps). (b) Copper(II)chloride, tert-butyl nitrite, acetonitrile, rt, $2 \mathrm{~h}$ (65\%). (c) $\mathrm{NaOH}, \mathrm{H}_{2} \mathrm{O} / \mathrm{THF}$ (1:1), rt, 18 h (quant). (d) (i) Oxalyl chloride, catalytic DMF, DCM, rt, 3 h; (ii) $\mathrm{AlCl}_{3}$, DCM, rt, $18 \mathrm{~h}$ (83\% over two steps). (e) (i) 31, $n$-butyllithium, THF, $-78{ }^{\circ} \mathrm{C}, 30 \mathrm{~min}$; (ii) $82,-78{ }^{\circ} \mathrm{C}$ to $\mathrm{rt}, 1 \mathrm{~h}(79 \%)$. (f) Acetic acid/1,4-dioxane $(1: 1), 140{ }^{\circ} \mathrm{C}, 10 \mathrm{~min}(58 \%)$. (g) (i) N,O-Bis(trimethylsilyl)trifluoroacetamide, DCM, reflux, $18 \mathrm{~h}$; (ii) iodomethane, $50{ }^{\circ} \mathrm{C}, 24 \mathrm{~h}(61 \%)$. (h) Phenylboronic acid, $\mathrm{Na}_{2} \mathrm{CO}_{3}, 1 \mathrm{~mol} \%$ bis(triphenylphosphine) palladium(II)chloride, 1,4-dioxane, water, microwave irradiation, $150{ }^{\circ} \mathrm{C}, 5 \mathrm{~min}(68 \%)$. (i) Lawesson's reagent, 1,4-dioxane, reflux, $18 \mathrm{~h}(76 \%)$. (j) $\mathrm{R}_{1} \mathrm{R}_{2} \mathrm{NH}, 1,4$-dioxane, microwave irradiation, $100{ }^{\circ} \mathrm{C}, 4 \mathrm{~h}(51-92 \%)$. (k) Lawesson's reagent, 1,4-dioxane, reflux, $18 \mathrm{~h}$ (44-88\%). (l) (i) N,O-Bis(trimethylsilyl)trifluoroacetamide, 1,2-dichloroethane, reflux, $18 \mathrm{~h}$; (ii) ethyl 2-(bromomethyl)thiazole-4-carboxylate, reflux, $48 \mathrm{~h}$ (91\%). (m) 2-Methoxyethyl amine, triethylamine, 1,4-dioxane, reflux, $72 \mathrm{~h}$ (59\%). (n) Lawesson's reagent, 1,4-dioxane, reflux, $18 \mathrm{~h}$ (81\%). (o) (i) $\mathrm{NaOH}$, methanol/ $\mathrm{H}_{2} \mathrm{O}, \mathrm{reflux}, 1 \mathrm{~h}(91 \%)$; (ii) 5-aminotetrazole, benzotriazol-1-yl-oxytripyrrolidinophosphonium hexafluorophosphate, DMF, DIPEA, rt, $2 \mathrm{~h}(34 \%)$.

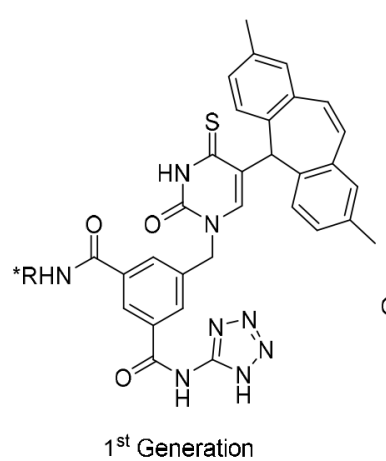

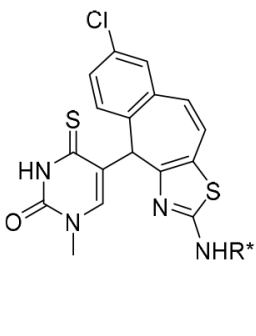<smiles></smiles>

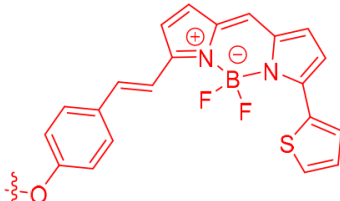

bodipy $\mathrm{A}$

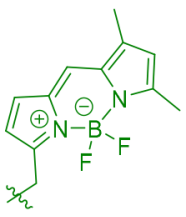

bodipy $B$

Figure 3. Design of the fluorescent $\mathrm{P} 2 \mathrm{Y}_{2} \mathrm{R}$ antagonists based on the SARs of 1 . The figure shows the potential attachment points to which either BODIPY A or BODIPY B could be attached via a suitable linking group. The asterisks $(*)$ represent the attachment points for the fluorophore linkage via a suitable linking group.

(2-(2-aminoethoxy)ethyl)carbamate to generate the corresponding amides (74-77). The Boc protecting groups were removed using TFA, and the resulting amines were coupled with the appropriate commercially available BODIPY succinimidyl ester
(SE), generating a small library of 14 fluorescent conjugates (78-91). These fluorescent conjugates were initially tested using the aforementioned NanoBRET binding assay at fixed concentrations of $10 \mu \mathrm{M}$ in the presence or absence of $10 \mu \mathrm{M} 1$ 


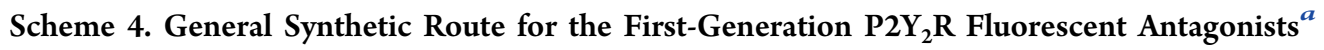

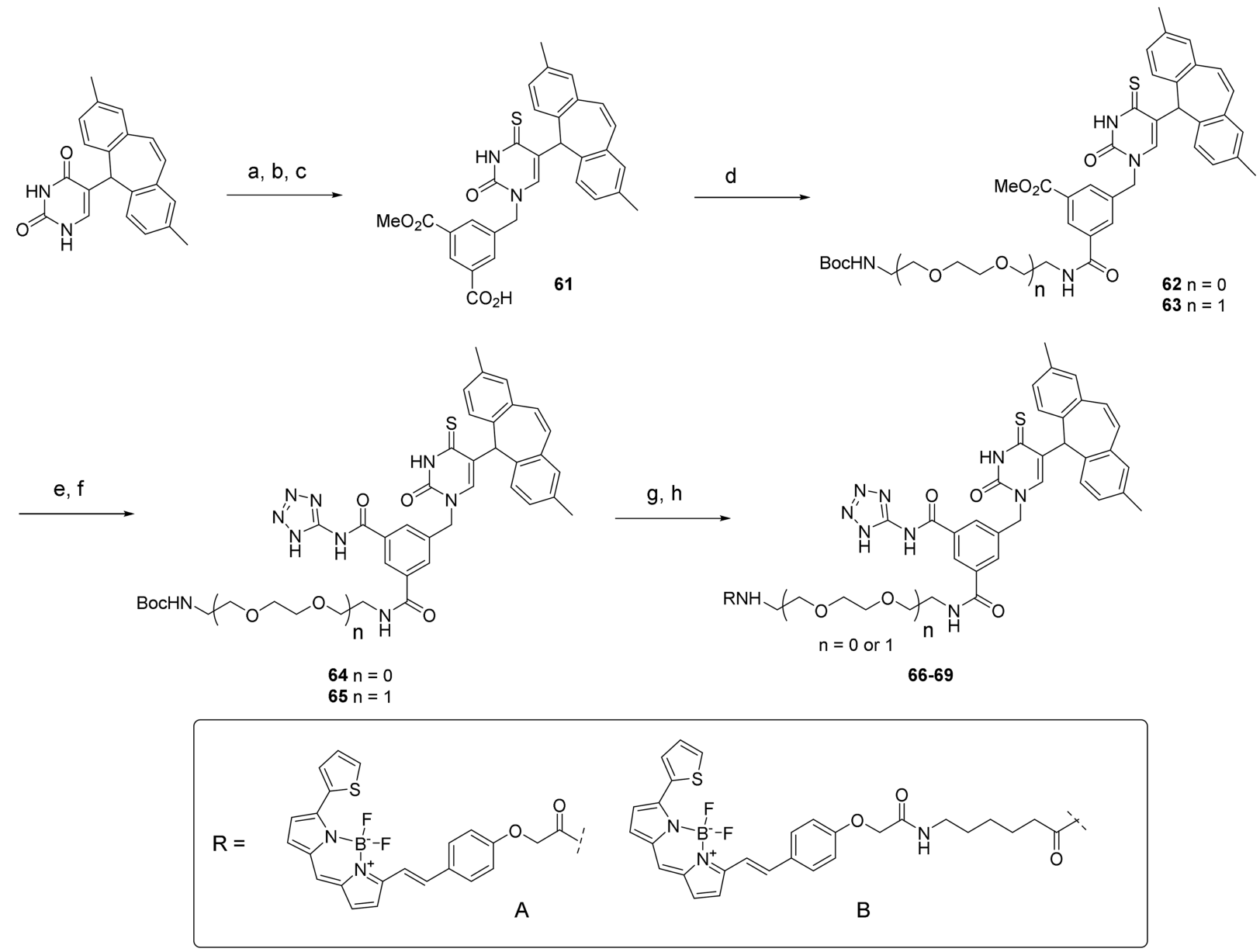

${ }^{a}$ Reagents and conditions: (a) Dimethyl 5-(bromomethyl)isophthalate, BSTFA, 1,2-dichloroethane, reflux, 16 h. (b) Lawesson's reagent, 1,4-dioxane, reflux, 16 h. (c) $\mathrm{NaOH}$ (2 equiv), $\mathrm{MeOH}$, toluene, $\mathrm{H}_{2} \mathrm{O}, 4 \mathrm{~h}, 65 \%$ (three steps). (d) tert-Butyl (2-(2-aminoethoxy)ethyl)carbamate (53\%) or tert-butyl (2-aminoethyl)carbamate (63\%), $\mathrm{NEt}_{3}$, benzotriazol-1-yl-oxytripyrrolidinophosphonium hexafluorophosphate, DMF, rt, $0.5 \mathrm{~h} .(\mathrm{e}) \mathrm{LiOH}$, $\mathrm{MeOH}, \mathrm{H}_{2} \mathrm{O}, 16 \mathrm{~h}, \mathrm{rt}, 14-30 \%$. (f) 4-Aminotetrazole, $\mathrm{NEt}_{3}$, benzotriazol-1-yl-oxytripyrrolidinophosphonium hexafluorophosphate, DMF, $1 \mathrm{~h}$, rt. (g) $\mathrm{HCl}(4 \mathrm{M})$ in 1,4-dioxane, $1 \mathrm{~h}$. (h) BODIPY-SE, DIPEA, DMF, rt, $1-4 \mathrm{~h}, 15-45 \%$.

Table 4. Affinities of 66, 67, 68, and 69 Determined in 1321N1 Astrocytoma Cells Expressing Recombinant NLuc-P2Y 2 R

\begin{tabular}{cccc|}
\hline example & $n^{a}$ & $\mathrm{R}^{b}$ & NanoBRET $\mathrm{p} K_{\mathrm{d}}{ }^{c}$ \\
66 & 0 & $\mathrm{~A}$ & $5.56 \pm 0.1(3)$ \\
67 & 0 & $\mathrm{~B}$ & $5.78 \pm 0.18(3)$ \\
68 & 1 & $\mathrm{~A}$ & $6.12 \pm 0.07(3)$ \\
69 & 1 & $\mathrm{~B}$ & $6.07 \pm 0.15(3)$
\end{tabular}

${ }^{a}$ See Scheme $4 .{ }^{b} \mathrm{~A}$ and $\mathrm{B}$ correspond to the $\mathrm{R}$ groups in Scheme 4. ${ }^{c} \mathrm{The} \mathrm{p} K_{\mathrm{d}}$ values were derived from saturation binding curves. The data shown are the means \pm SEM, and the numbers of separate experiments are given in parentheses.

(see Figure $\mathrm{S} 1$ in the Supporting Information). It was found that 80, 85, 86, and 87 generated the largest specific NanoBRET signals; therefore, their affinities were determined from saturation binding assays, demonstrating the excellent signal-to-noise ratios observed from NanoBRET assays even for low-affinity conjugates (Figure 4 and Table 5).

Reassuringly, the affinities determined for the nonfluorescent $\mathrm{P}_{2} \mathrm{Y}_{2} \mathrm{R}$ antagonist, 1 , with either $86\left(\mathrm{p} K_{\mathrm{i}}=7.45 \pm 0.13, n=4\right)$ or $87\left(\mathrm{p} K_{\mathrm{i}}=7.32 \pm 0.13, n=4\right)$ were consistent with the affinities determined using the $\mathrm{P}_{2} \mathrm{Y}_{2} \mathrm{R}$ functional assay $\left(\mathrm{p} K_{\mathrm{b}}=7.51 \pm 0.09\right)$, demonstrating that the $\mathrm{P} 2 \mathrm{Y}_{2} \mathrm{R}$ fluorescent ligands could be used in a NanoBRET assay for determining the affinities of nonfluorescent $\mathrm{P}_{2} \mathrm{Y}_{2} \mathrm{R}$ antagonists. The clear demonstration of the saturable specific binding of these low-affinity fluorescent ligands confirmed the utility of the NanoBRET binding format and the ability to exploit the good signal-tonoise ratio of this proximity-based assay. To explore the opportunity to develop higher-affinity fluorescent conjugates, we embarked on a synthetic strategy to incorporate affinityenhancing acyl-tetrazole functional groups into the fluorescent compounds.

Third-Generation $\mathrm{P} 2 \mathrm{Y}_{2} \mathrm{R}$ Fluorescent Ligands. The general synthetic route for the third-generation $\mathrm{P}_{2} \mathrm{Y}_{2} \mathrm{R}$ fluorescent antagonists is illustrated in Scheme 6. The displacement of the chlorine atom in compound $\mathbf{5 7}$ with tert-butyl 3-aminopropanoate gave the tert-butyl ester, 92. Treatment with TFA afforded the conversion of the tert-butyl ester to the corresponding acid, which was immediately activated and coupled to tert-butyl (2-aminoethyl)carbamate, 93, with HATU. Hydrolysis of the ethyl ester, activation of the carboxylic acid by benzotriazol-1-yl-oxytripyrrolidinophosphonium hexafluorophosphate, and coupling with 5-aminotetrazole gave the amidotetrazole, 94. The Boc protecting group was removed, 
Scheme 5. General Synthetic Route for the Second-Generation $P 2 Y_{2} R$ Fluorescent Antagonists ${ }^{a}$

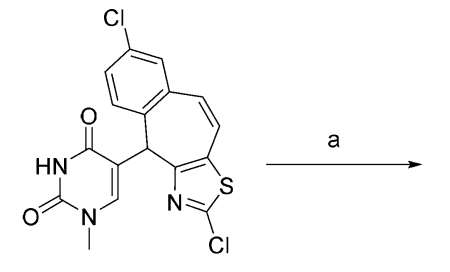

43

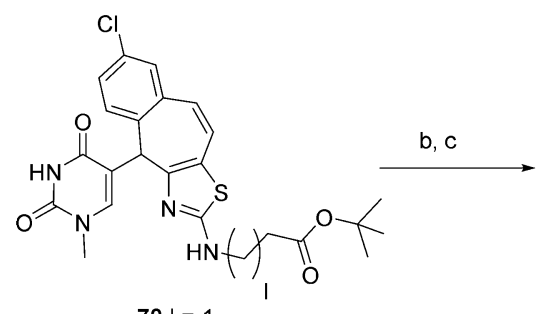

$701=1$

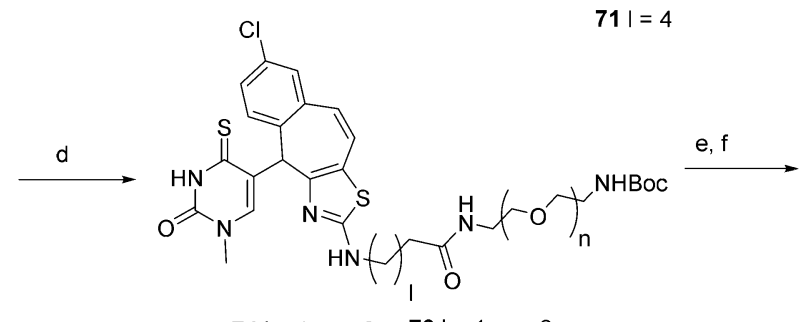

$74 I=1, n=0 \quad 76 I=1, n=2$

$75 \mathrm{I}=4, \mathrm{n}=0 \quad 77 \mathrm{I}=4, \mathrm{n}=2$

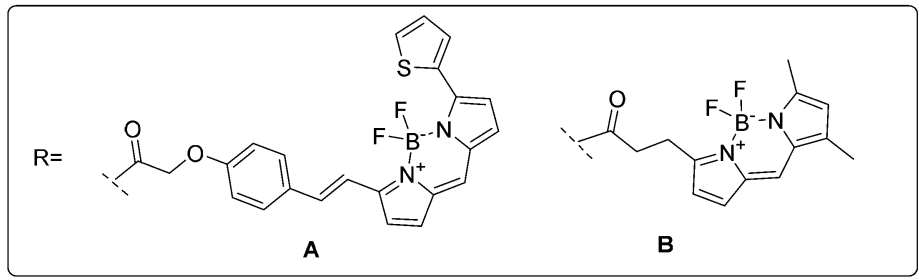

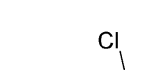

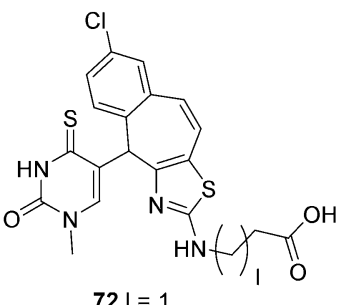

$731=4$

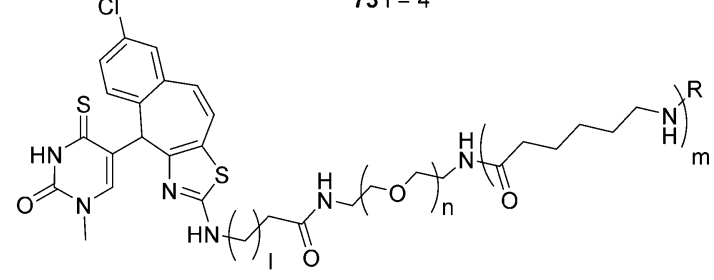

78-91

$\begin{array}{llllllllll}\text { Example } & \mathrm{I} & \mathrm{n} & \mathrm{m} & \mathrm{R} & \text { Example } & \mathrm{I} & \mathrm{n} & \mathrm{m} & \mathrm{R} \\ \mathbf{7 8} & 1 & 0 & 0 & \mathrm{~A} & \mathbf{8 5} & 1 & 0 & 0 & \mathrm{~B} \\ \mathbf{7 9} & 1 & 0 & 1 & \mathrm{~A} & \mathbf{8 6} & 1 & 0 & 1 & \mathrm{~B} \\ \mathbf{8 0} & 1 & 2 & 1 & \mathrm{~A} & \mathbf{8 7} & 1 & 2 & 1 & \mathrm{~B} \\ \mathbf{8 1} & 4 & 0 & 0 & \mathrm{~A} & \mathbf{8 8} & 4 & 0 & 0 & \mathrm{~B} \\ \mathbf{8 2} & 4 & 2 & 0 & \mathrm{~A} & \mathbf{8 9} & 4 & 2 & 0 & \mathrm{~B} \\ \mathbf{8 3} & 4 & 0 & 1 & \mathrm{~A} & \mathbf{9 0} & 4 & 0 & 1 & \mathrm{~B} \\ \mathbf{8 4} & 4 & 2 & 1 & \mathrm{~A} & \mathbf{9 1} & 4 & 2 & 1 & \mathrm{~B}\end{array}$

${ }^{a}$ Reagents and conditions: (a) Amine, $\mathrm{NEt}_{3}, 1$,4-dioxane, reflux, $72 \mathrm{~h}, 96-97 \%$. (b) Lawesson's reagent, 1,4-dioxane, $100{ }^{\circ} \mathrm{C}, 18 \mathrm{~h}, 39-74 \%$. (c) TFA, DCM, rt, 30 min. (d) Amine, benzotriazol-1-yl-oxytripyrrolidinophosphonium hexafluorophosphate, DMF, DIPEA, rt, $1 \mathrm{~h}, 36-58 \%$ (two steps). (e) TFA, DCM, rt, 30 min. (f) BODIPY-SE, DIPEA, DMF, rt, 1-4 h, 10-54\% (two steps).

and the resulting amine was attached to the fluorophore with the appropriate BODIPY succinimidyl ester (Scheme 6).

In contrast to the nonfluorophore compounds 14 and 24, whose affinities hardly changed when their uracil N1-substituents were changed, in three of the four compounds (95-98), there were significant increases in their affinities relative to those of the nonamidotetrazole series (compare 98 with 86, 96 with 85 , and 97 with 80; Tables 5 and 6).

Pharmacological Evaluation of Third-Generation $\mathrm{P} 2 \mathrm{Y}_{2} \mathrm{R}$ Fluorescent Ligands. To further evaluate the utility of the fluorescent ligands in studying the pharmacology of P2 $\mathrm{Y}_{2} \mathrm{R}$, one BODIPY A and one BODIPY B linked fluorescent ligand (97 and 98, respectively) were chosen for further studies. Initially, we confirmed that 97 and 98 still retained the ability to functionally antagonize $\mathrm{P}_{2} \mathrm{Y}_{2} \mathrm{R}$ (Figure 5). In a $\mathrm{Ca}^{2+}$-mobilization assay, modest rightwards shifts of the agonist-dose-response curves were observed for both $10 \mu \mathrm{M}$ $97\left(\mathrm{p} K_{\mathrm{b}}=5.69 \pm 0.05, n=8\right)$ and $10 \mu \mathrm{M} 98\left(\mathrm{p} K_{\mathrm{b}}=5.87 \pm\right.$ $0.05, n=7)$.

Compounds 97 and 98 bought about clear concentrationdependent increases in BRET ratios in NLuc-tagged- $h \mathrm{P}_{2} \mathrm{Y}_{2} \mathrm{R}$ assays (Figure 6). These increases were antagonized by 1 (Figure 6c,d), yielding pKi values of $7.66 \pm 0.11(n=9)$ and $7.38 \pm 0.04(n=6)$ for the antagonism of 97 and 98 binding, respectively.

To further evaluate the utility of fluorescent conjugate $\mathbf{9 8}$ in the NanoBRET ligand-binding assay, the affinities of a selection of $\mathrm{P}_{2} \mathrm{Y}_{2} \mathrm{R}$ antagonists $(1,3,6,22,23,60$, and 86$)$ and the previously reported stabilized-triphosphate $\mathrm{P}_{2} \mathrm{Y}_{2} \mathrm{R}$ antagonist, 99, ${ }^{8}$ over a range of $\mathrm{P} 2 \mathrm{Y}_{2} \mathrm{R}$ affinities, were determined in competition binding experiments.
All eight compounds induced concentration-dependent decreases in the specific binding of 98, which enabled their affinities to be determined. There was a good correlation between the values obtained in the NanoBRET assay and those determined in the $\mathrm{Ca}^{2+}$-mobilization assay (Table 7). In addition to those of the antagonists, the NanoBRET assay was also used to estimate the affinity of UTP $\gamma$ S. As there have been no reports of radio ligands for $\mathrm{P}_{2} \mathrm{Y}_{2} \mathrm{R}$, this measurement has not previously been possible.<smiles>O=c1[nH]c(=S)c(C2c3ccccc3CCc3ccccc32)cn1[C@@H]1OC(COP(=O)(O)OP(=O)(O)C(Cl)(Cl)P(=O)([O-])O)[C@@H](O)[C@H]1O</smiles>

The availability of both green (98) and red (97) fluorescent $\mathrm{P}_{2} \mathrm{Y}_{2} \mathrm{R}$ ligands with reasonable affinities for $h \mathrm{P} 2 \mathrm{Y}_{2} \mathrm{R}$ suggested that they may both have utility in imaging the receptor in living cells. Confocal-microscope images of fluorescent ligands 97 and 98 with $1321 \mathrm{~N} 1$ astrocytoma cells expressing $h \mathrm{P} 2 \mathrm{Y}_{2} \mathrm{R}$ (Figure $7 \mathrm{a}, \mathrm{c}$ ) showed localized membrane fluorescence and very little intracellular fluorescence. When the cells were pretreated with 1, the membrane-specific fluorescence of 97 and 98 was substantially reduced (Figure $7 \mathrm{~b}, \mathrm{~d}$ ), indicating that the majority of the membrane fluorescence observed was specific labeling of $\mathrm{P}_{2} \mathrm{Y}_{2} \mathrm{R}$. 
a)

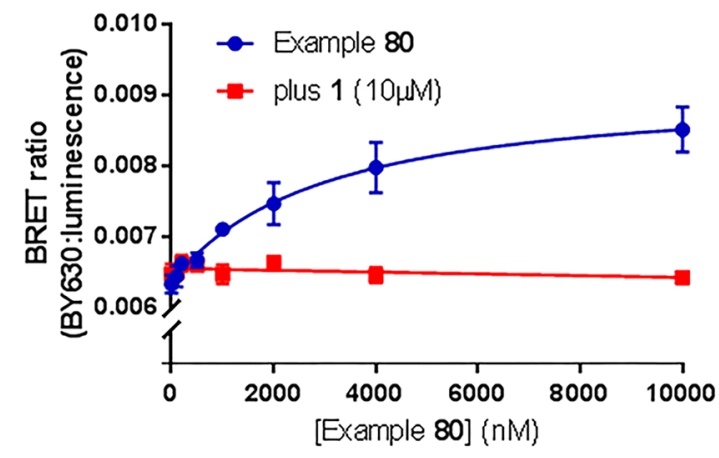

c)

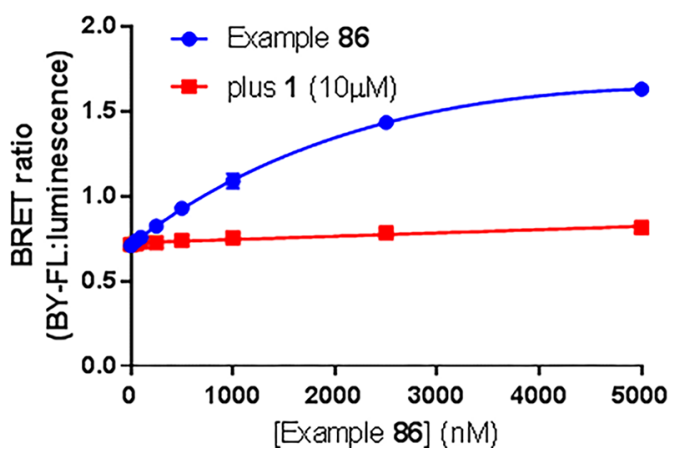

b)

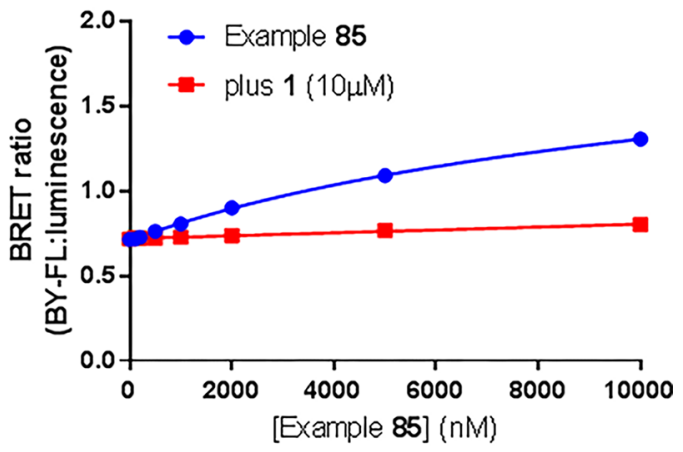

d)

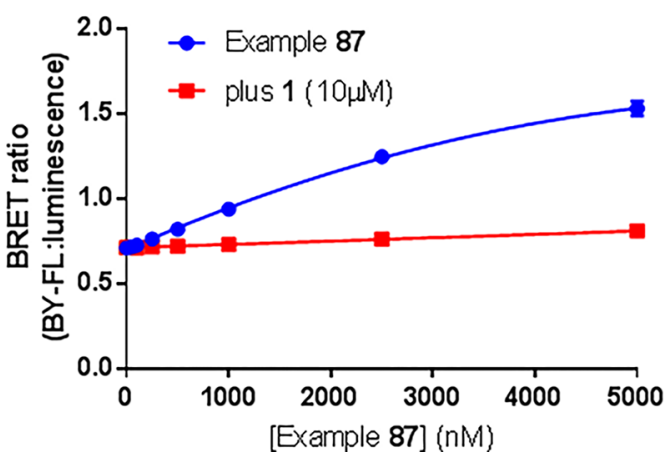

Figure 4. NanoBRET saturation binding isotherms, determined in $1321 \mathrm{~N} 1$ astrocytoma cells expressing recombinant $\mathrm{NLuc} \mathrm{P} 2 \mathrm{Y}_{2} \mathrm{R}$, for (a) 80, (b) 85 , (c) 86 , and (d) 87 in the absence and presence of $10 \mu \mathrm{M} \mathrm{1}$. The data points are the mean values \pm SEM $(n=3$ or 4$)$.

Table 5. Affinities of $85,86,87$, and 80 Determined in 1321N1 Astrocytoma Cells Expressing Recombinant NLuc-P2Y 2 R

$\begin{array}{cc}\text { example } & \mathrm{pK}_{\mathrm{d}}{ }^{a} \\ \mathbf{8 0} & 5.29 \pm 0.17(4) \\ \mathbf{8 5} & 4.91 \pm 0.14(4) \\ \mathbf{8 6} & 5.67 \pm 0.10(4) \\ \mathbf{8 7} & 5.38 \pm 0.19(3)\end{array}$

${ }^{a_{T}}$ The $\mathrm{p} K_{\mathrm{d}}$ values were derived from saturation binding curves. The data shown are the means \pm SEM, and the numbers of separate experiments are given in parentheses.

\section{CONCLUSION}

We have described the synthesis and evaluation of new examples of acidic $h \mathrm{P} 2 \mathrm{Y}_{2} \mathrm{R}$ antagonists based on the known $h \mathrm{P} 2 \mathrm{Y}_{2} \mathrm{R}$ antagonist, 1 . In addition, we have shown the discovery of a new series of neutral $h \mathrm{P}_{2} \mathrm{Y}_{2} \mathrm{R}$ antagonists and demonstrated SAR leading to the identification of potent $h P 2 Y_{2} R$ antagonists (such as $\mathbf{2 0}$ and 23). In addition, we have shown a stereochemical preference for biological activity within this series, as typified by the resolved examples of $\mathbf{2 0}$ versus $\mathbf{2 1}$ and 22 versus 23 . Vibrational circular dichroism has suggested that in the case of 16 , all of the $h \mathrm{P}_{2} \mathrm{Y}_{2} \mathrm{R}$ biological activity resides in the $(R)$-enantiomer (23), although single-crystal X-ray work will be required to confirm this initial stereochemical assignment. The SAR studies led to the identification of suitable linking sites for the attachment of the fluorescent ligands, thus generating three distinct series of fluorescently labeled $h \mathrm{P} 2 \mathrm{Y}_{2} \mathrm{R}$ antagonists. From this extensive synthetic work, two examples (97 and 98) were identified as demonstrating both functional antagonist activities (in $\mathrm{Ca}^{2+}$-mobilization assays) and sufficient affinities for $h \mathrm{P} 2 \mathrm{Y}_{2} \mathrm{R}$ through a new bioluminescenceresonance-energy-transfer (BRET) assay. In addition, confocal microscopy revealed clear, displaceable membrane labeling of astrocytoma cells expressing $h \mathrm{P}_{2} \mathrm{Y}_{2} \mathrm{R}$. These excellent imaging properties make 97 and 98 ideal tools for studying $h \mathrm{P} 2 \mathrm{Y}_{2} \mathrm{R}$ distribution and organization. Finally, the discovery of the new $h \mathrm{P} 2 \mathrm{Y}_{2} \mathrm{R}$-antagonist fluorescent ligands (97 and 98) became realized as a result of an extensive program of synthetic chemistry, in which it proved essential to explore the parallel changes of linker-attachment points, fluorophores, and linking groups. ${ }^{30}$ From this study, only a few fluorescent conjugates were shown to possess sufficient affinities to enable the establishment of a new NanoBRET-based fluorescent assay for the identification of new $h \mathrm{P} 2 \mathrm{Y}_{2} \mathrm{R}$ fragments and ligands.

\section{EXPERIMENTAL SECTION}

Chemistry General Methods. Chemicals and solvents were provided by Fisher Scientific UK, Acros Organics, Sigma-Aldrich, Merck Millipore, or Fluorochem. BODIPYFL-X-NHS (D6102) and BODIPY630/650-X-NHS (D10000) were purchased from Molecular Probes (Invitrogen). All reactions were monitored by TLC using Merck Silica Gel $60 \AA$ A F254 TLC plates or by LC-MS. Unless otherwise stated, all compounds were dried under high vacuum either at $\mathrm{rt}$ or in an oven at $40{ }^{\circ} \mathrm{C}$. LC-MS data was collected on a Shimadzu UFLCXR HPLC system coupled to an Applied Biosystems API 2000 electrospray-ionization (ESI) LC-MS/MS. The column used was a Phenomenex Gemini-NX $(3 \mu \mathrm{m}, 110 \AA, \mathrm{C} 18,50 \times 2 \mathrm{~mm})$, and it was operated at $40{ }^{\circ} \mathrm{C}$. The flow rate was $0.5 \mathrm{~mL} / \mathrm{min}$, and UV detection was at 220 and $254 \mathrm{~nm}$. LC-MS method 1 was $5 \%$ B for $1 \mathrm{~min}, 5-98 \%$ B over 2 min, $98 \%$ B for 2 min, $98-5 \%$ B over $0.5 \mathrm{~min}$, and then $5 \%$ B for $1 \mathrm{~min}$. LC-MS method 2 was $10 \%$ B for $1.5 \mathrm{~min}, 10-98 \%$ B over 


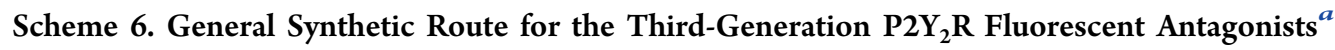<smiles></smiles>

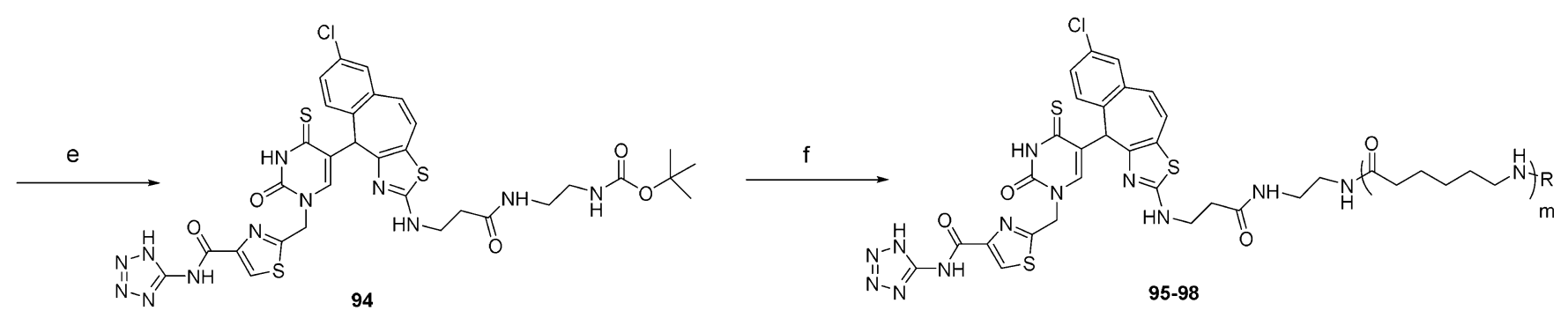<smiles></smiles>

$\mathrm{m}=0$ or 1

${ }^{a}$ Reagents and conditions: (a) Amine, $\mathrm{NEt}_{3}, 1$ 1,4-dioxane, reflux, $72 \mathrm{~h}$ (89\%). (b) Lawesson's reagent, 1,4-dioxane, reflux, $18 \mathrm{~h}$ (48\%). (c) TFA, DCM, rt, 30 min (55\%). (d) tert-Butyl (2-aminoethyl)carbamate, HATU, DCM, DIPEA, rt, 24 h (63\%). (e) (i) NaOH, MeOH, reflux, 4 h; (ii) 5-aminotetrazole, benzotriazol-1-yl-oxytripyrrolidinophosphonium hexafluorophosphate, DIPEA, DMF, rt, $4 \mathrm{~h}$ (28\% over two steps). (f) (i) TFA, DCM, rt, $30 \mathrm{~min}$; (ii) BODIPY-SE, DIPEA, DMF, rt, 1-4 h (11-18\% over two steps). The structures of compounds $95-98$ are assigned in Table 6.

Table 6. Affinities of 95, 96, 97, and 98 Determined in 1321N1 Astrocytoma Cells Expressing Recombinant NLuc-P2Y 2 R

$\begin{array}{cccc}\text { example } & m^{a} & \mathrm{R}^{b} & \text { NanoBRET } \mathrm{p}_{\mathrm{d}}{ }^{c} \\ 95 & 0 & \mathrm{~A} & 6.05 \pm 0.12(3) \\ 96 & 0 & \mathrm{~B} & 7.05 \pm 0.05(3) \\ 97 & 1 & \mathrm{~A} & 6.89 \pm 0.06(7) \\ 98 & 1 & \mathrm{~B} & 6.32 \pm 0.10(17)\end{array}$

${ }^{a}$ See Scheme 6. ${ }^{b} \mathrm{~A}$ and $\mathrm{B}$ correspond to the $\mathrm{R}$ groups in Scheme 6. ${ }^{c}$ The $\mathrm{p} K_{\mathrm{d}}$ values were derived from saturation binding curves. The data shown are the means \pm SEM, and the numbers of separate experiments are given in parentheses.
8 min, $98 \%$ B for $2 \min , 98-10 \%$ B over $0.5 \mathrm{~min}$, and then $10 \%$ B for $1 \mathrm{~min}$. Solvent A was $0.1 \%$ formic acid in water, and solvent B was acetonitrile. Unless otherwise stated, the compounds reported had purities $>95 \%$ at the wavelength and method quoted. HRMS data was collected on a Bruker microTOF II mass spectrometer using electrospray ionization (ESI-TOF). Adducts within errors of $\pm 10 \mathrm{ppm}$ were reported. Preparative RP-HPLC was performed on a Waters 2767 sample manager coupled to Waters 2525 binary-gradient module and a Waters 2457 dual-wavelength absorbance detector. The column used was a Phenomenex Gemini-NX $(5 \mu \mathrm{m}, 110 \AA, \mathrm{C} 18,150 \times 21 \mathrm{~mm})$ at ambient temperature. The flow rate was $40 \mathrm{~mL} / \mathrm{min}$, and UV detection was at $254 \mathrm{~nm}$. Preparative-RP-HPLC method 3 was $10 \%$ B for $1 \mathrm{~min}, 10-35 \%$ B over $4 \mathrm{~min}, 35-40 \%$ B over $20 \mathrm{~min}, 40-90 \%$ B over $2 \mathrm{~min}, 90-10 \% \mathrm{~B}$ over $2 \mathrm{~min}$, and then $10 \% \mathrm{~B}$ for $1 \mathrm{~min}$.
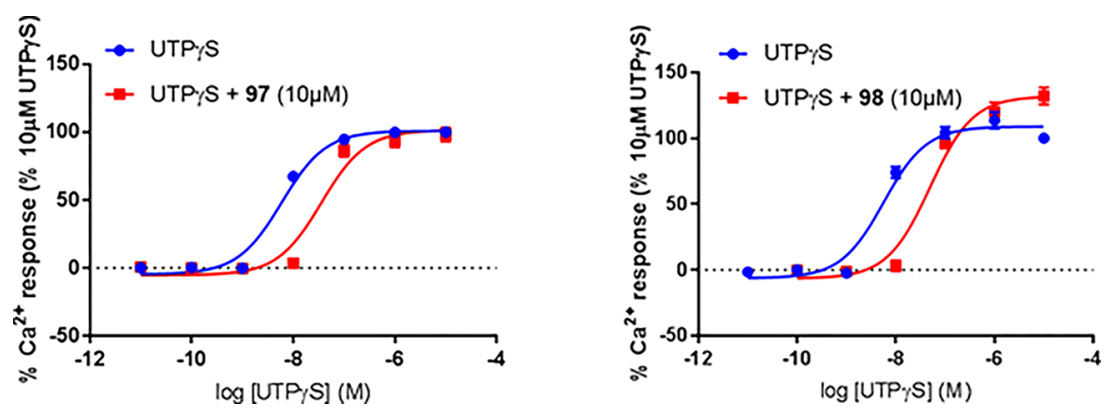

Figure 5. Pharmacological evaluations of 97 and 98 showing the effects on $\mathrm{Ca}^{2+}$ mobilization in $h \mathrm{P} 2 \mathrm{Y}_{2} \mathrm{R}-1321 \mathrm{~N} 1$ cells induced by $0.1 \mu \mathrm{M}$ UTP $\gamma \mathrm{S}$ $(n=7$ or 8$)$. 
a)

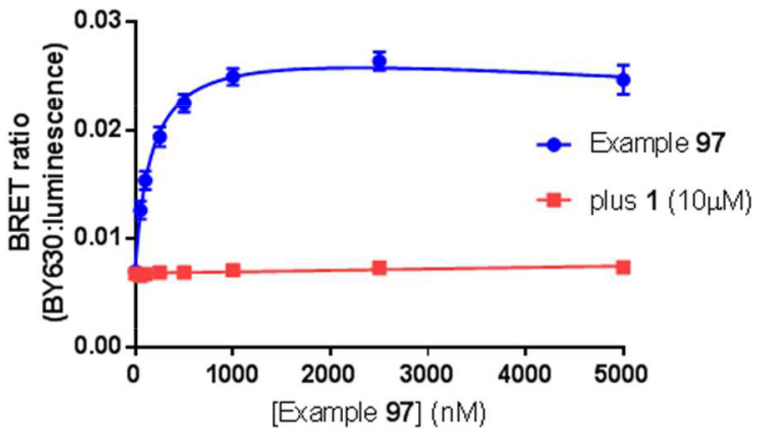

b)

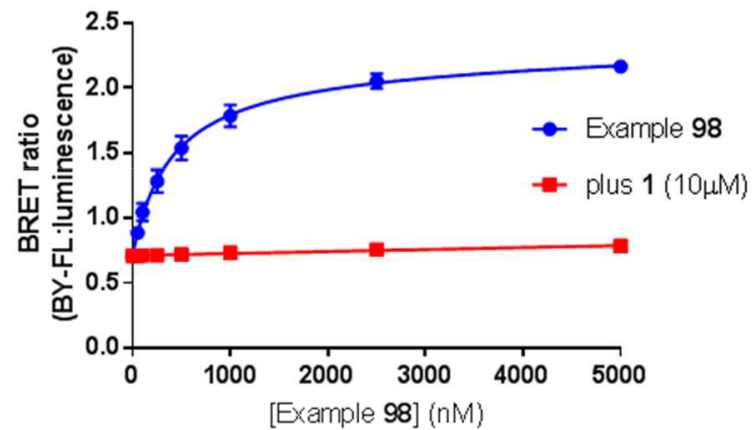

c)

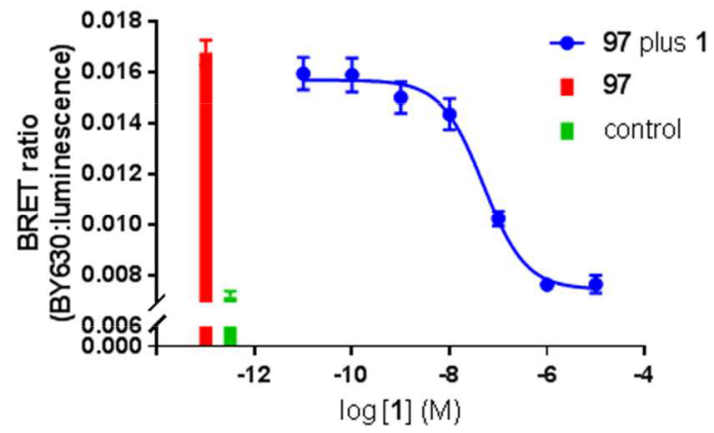

d)

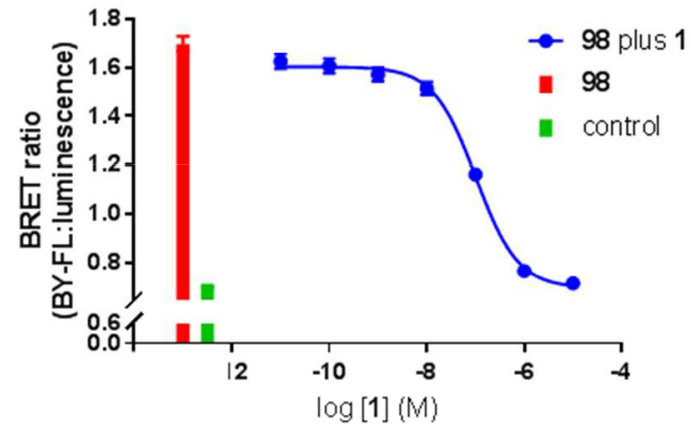

Figure 6. Pharmacological evaluations of (a) 97 and (b) 98 showing BRET saturation binding $(n=7)$. Displacement of (c) 97 (100 nM, $n=9)$ and (d) $98(1 \mu \mathrm{M}, n=6)$ binding in NLuc-P2Y $21321 \mathrm{~N} 1$ cells by 1 . The values shown are the means \pm SEM.

Table 7. Comparison of the Affinity Estimates Obtained in the $\mathrm{Ca}^{2+}$-Mobilization and NanoBRET Assays ${ }^{a}$

$\begin{array}{lcc}\text { example } & \mathrm{pK}_{\mathrm{b}} \mathrm{Ca}^{2+} \text { mobilization }^{b} & \mathrm{p} K_{\mathrm{i}} \text { NanoBRET }(98)^{c} \\ \mathbf{1} & 7.51 \pm 0.09(12) & 7.38 \pm 0.04(6) \\ 3 & 7.11 \pm 0.14(7) & 6.89 \pm 0.05(6) \\ 6 & 5.99 \pm 0.03(7) & 5.81 \pm 0.10(6) \\ 22 & <5.0(6) & 5.28 \pm 0.05(3) \\ \mathbf{2 3} & 6.74 \pm 0.10(6) & 6.56 \pm 0.16(3) \\ 60 & 6.53 \pm 0.04(7) & 6.84 \pm 0.02(6) \\ 86 & 6.30 \pm 0.05(6) & 6.40 \pm 0.01(6) \\ 99 & 6.59 \pm 0.12(3)^{d} & 7.08 \pm 0.03(3) \\ \mathrm{UTP} \gamma \mathrm{S} & \mathrm{ND}^{e} & 5.46 \pm 0.04(6)\end{array}$

${ }^{a_{T}}$ The data shown are the means \pm SEM, and the numbers of separate experiments are given in parentheses. ${ }^{b}$ The estimated affinity value for each antagonist $\left(\mathrm{p} K_{\mathrm{b}}\right)$ was calculated using the Gaddum equation from the shift of the agonist-dose-response curve brought about by the addition of a single concentration of the antagonist. ${ }^{c}$ Measured in competition binding experiments with fluorescent ligand 98 . ${ }^{d}$ Reported $\mathrm{pA}_{2}=8.0 .^{8}{ }^{e} \mathrm{ND}=$ not determined due to agonist activity.

Preparative-RP-HPLC method 4 was $10 \%$ B for $1 \mathrm{~min}, 10-45 \%$ B over $4 \mathrm{~min}, 45-50 \%$ B over $20 \mathrm{~min}, 50-90 \%$ B over $2 \mathrm{~min}, 90-10 \%$ $\mathrm{B}$ over $2 \mathrm{~min}$, and then $10 \% \mathrm{~B}$ for $1 \mathrm{~min}$. Solvent A was $0.1 \%$ trifluoroacetic acid in water, and solvent $\mathrm{B}$ was $0.1 \%$ trifluoroacetic acid in acetonitrile. Chiral-HPLC was performed on a Dionex ICS-3000 SP single pump coupled to a Rheodyne 9725i PEEKinjector with a Dionex UltiMate 3000 Variable Wavelength Detector. The columns used for the analytical and semipreparative runs were $250 \times 4.6 \mathrm{~mm}$ and $250 \times 10 \mathrm{~mm}$ Phenomenex Luxes $(5 \mu \mathrm{m}$, Amylose-2), respectively. These operated at ambient temperature, and unless otherwise stated, the mobile phase was $25 \%$ ethanol/hexane with a flow rate of $1 \mathrm{~mL} / \mathrm{min}$ for the analytical runs and $5 \mathrm{~mL} / \mathrm{min}$ for the semipreparative runs. UV detection was at $254 \mathrm{~nm}$. NMR spectroscopy was performed

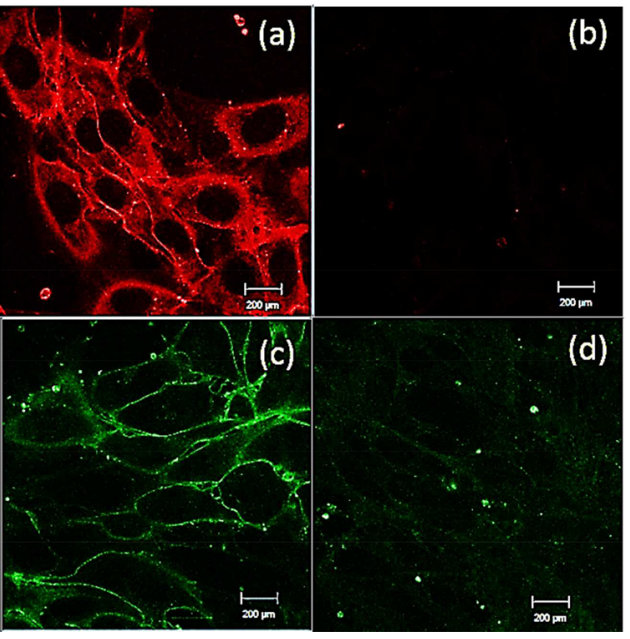

Figure 7. Visualization of the binding of (a,b) 97 and (c,d) 98 on astrocytoma cells expressing $h \mathrm{P}_{2} \mathrm{Y}_{2} \mathrm{R}$. The images in the left-hand column $(\mathrm{a}, \mathrm{c})$ are confocal image with $100 \mathrm{nM}$ concentrations of the fluorescent ligands, whereas the right-hand column $(\mathrm{b}, \mathrm{d})$ shows images of the cells with the fluorescent ligands and $10 \mu \mathrm{M} 1$. In all of the conditions, the cells were incubated for $30 \mathrm{~min}$ at $37^{\circ} \mathrm{C}$ in the presence or absence of $10 \mu \mathrm{M} 1$. Single-equatorial confocal images were then obtained in the continued presence of the fluorescent ligand (97 or 98) and the unlabeled antagonist. The images shown are from a single experiment representative of the four performed.

with a Bruker AV(III) HD 400 NMR spectrometer equipped with a $5 \mathrm{~mm} \mathrm{BBFO}+$ probe, which recorded the ${ }^{1} \mathrm{H}$ and ${ }^{13} \mathrm{C}$ NMR at 400.25 and $100.66 \mathrm{MHz}$, respectively, or with a Bruker AV(III) $500 \mathrm{NMR}$ spectrometer equipped with a $5 \mathrm{~mm}$ dual ${ }^{1} \mathrm{H} /{ }^{13} \mathrm{C}$ helium-cooled cryoprobe, which recorded the ${ }^{1} \mathrm{H}$ and ${ }^{13} \mathrm{C}$ NMR at 500.13 and $125.77 \mathrm{MHz}$, 
respectively. The NMR data was processed using iNMR (version 5.5.7), which referenced the spectra to those of the residual solvents. Chemical shifts $(\delta)$ are quoted as values in parts per million, and coupling constants $(J)$ are given in hertz. Multiplicities are described using the following abbreviations: $s$, singlet; $d$, doublet; $t$, triplet; q, quartet; qi, quintet; sep, septet; m, multiplet; app, apparent; and br, broad.

All compounds submitted for biological screening had purities $>95 \%$.

General Procedure 1: Substitution of 2-Chlorothiazole to Generate 2-Aminothiazoles. A stirred solution of 43 (0.15$0.50 \mathrm{mmol})$ dissolved in anhydrous 1,4-dioxane $(0.1 \mathrm{M}, 1.5-5.0 \mathrm{~mL})$ was treated with a chosen amine (4 equiv, $0.45-2.00 \mathrm{mmol}$ ) and triethyl amine (10 equiv, $1.50-5.00 \mathrm{mmol}$ ) under $\mathrm{N}_{2}$ and either heated to reflux for 36-72 h until completion was observed by TLC or LC-MS or heated in a microwave reactor at $100{ }^{\circ} \mathrm{C}$ for $4 \mathrm{~h}$. The reaction mixture was concentrated in vacuo directly onto a silica gel and purified by chromatography on a silica gel $(2-5 \% \mathrm{MeOH} / \mathrm{DCM}$ with $0.1 \% 880 \mathrm{NH}_{3}$ ) to afford the desired 2-aminothiazoles, 45-56.

General Procedure 2: Thionation of Uracils to 4-Thiouracils Using Lawesson's Reagent. Stirred suspensions or solutions of the uracils $(0.05-0.25 \mathrm{mmol})$ and Lawesson's reagent (2 stoichiometric equiv/1 molar equiv, $0.05-0.25 \mathrm{mmol}$ ) in anhydrous 1,4-dioxane $(0.05 \mathrm{M}, 1-5 \mathrm{~mL})$ were heated to reflux $\left(120^{\circ} \mathrm{C}\right)$ under $\mathrm{N}_{2}$ for $18 \mathrm{~h}$, generating deep-yellow-orange solutions. These were allowed to cool to room temperature, and the crude reaction mixtures were concentrated in vacuo onto silica gels, and unless otherwise stated, the title compounds were purified by chromatography on silica gels and eluted with $0-5 \% \mathrm{MeOH} / \mathrm{DCM}$ to afford the desired 4-thiouracils.

General Procedure 3: Synthesis of BODIPY-Labeled Fluorescent Conjugates. A solution of a Boc-protected amine $(2.00 \mu \mathrm{mol})$ dissolved in DCM $(0.50 \mathrm{~mL})$ was treated with TFA $(0.25 \mathrm{~mL})$ and stirred at $\mathrm{rt}$ for $30 \mathrm{~min}$. This was then diluted with toluene $(10 \mathrm{~mL})$ and concentrated in vacuo to one-fifth the volume; this was repeated three times before the solution was concentrated to dryness, affording the TFA salt of the conjugate. This was dissolved in DMF $(500 \mu \mathrm{L})$, treated with DIPEA $(25 \mu \mathrm{L})$ and then with a solution of BODIPYFLX-NHS $(1.00 \mathrm{mg}, 2.00 \mu \mathrm{mol})$ in DMF $(150 \mu \mathrm{L})$, BODIPY630/650-XNHS $(1.32 \mathrm{mg}, 2.00 \mu \mathrm{mol})$ in DMF $(150 \mu \mathrm{L})$, BODIPYFL-NHS, or BODIPY630/650-NHS; the latter two were generated in situ by mixing BODIPYFL- $\mathrm{CO}_{2} \mathrm{H} \mathrm{X}(0.78 \mathrm{mg}, 2.00 \mu \mathrm{mol})$ or BODIPY630/ $650-\mathrm{CO}_{2} \mathrm{H}(0.90 \mathrm{mg}, 2.00 \mu \mathrm{mol})$ with HATU $(0.76 \mathrm{mg}, 2.00 \mu \mathrm{mol})$, NHS $(0.23 \mathrm{mg}, 2.00 \mu \mathrm{mol})$, and DIPEA $(25 \mu \mathrm{L})$ in DMF $(150 \mu \mathrm{L})$ for $30 \mathrm{~min}$ before they were added. The solution was then allowed to react at room temperature in the absence of light for $1-4 \mathrm{~h}$ until completion was observed by LC-MS. The desired fluorescent conjugate was then purified directly from the reaction mixture by preparative reversephase HPLC and lyophilization.

2-((5-(2,8-Dimethyl-5H-dibenzo[a,d][7]annulen-5-yl)-2-oxo-4-thioxo-3,4-dihydropyrimidin-1(2H)-yl)methyl)- $\mathrm{N}$-(1H-tetrazol-5-yl)thiazole-4-carboxamide (3). PyBroP $(0.046 \mathrm{~g}, 0.1 \mathrm{mmol})$ was added to a stirred solution of 2-((5-(2,8-dimethyl-5H-dibenzo[a,d][7]annulen5-yl)-2-oxo-4-thioxo-3,4-dihydropyrimidin-1(2H)-yl)methyl)thiazole-4carboxylic acid $(2,0.04 \mathrm{~g}, 0.082 \mathrm{mmol}), 5$-aminotetrazole monohydrate $(0.018 \mathrm{~g}, 0.18 \mathrm{mmol})$, and triethylamine $(0.036 \mathrm{~g}, 0.36 \mathrm{mmol})$ in DMF $(1 \mathrm{~mL})$. After $10 \mathrm{~min}$, the reaction mixture was partitioned between $1 \mathrm{M}$ hydrochloric acid and ethyl acetate. The ethyl acetate solution was washed with water and brine and evaporated to dryness. Purification was by silica-gel chromatography eluted with ethyl acetate/methanol/ acetic acid (93:5:2). Yield: $0.025 \mathrm{~g}, 0.049 \mathrm{mmol}, 49 \%$ (yellow solid). ${ }^{1} \mathrm{H}$ NMR (400 MHz, DMSO) $\delta: 12.73(\mathrm{~s}, 1 \mathrm{H}), 12.17$ (s, 1H), 11.96 (s, $1 \mathrm{H}), 8.74(\mathrm{~s}, 1 \mathrm{H}), 7.46(\mathrm{~d}, J=7.8 \mathrm{~Hz}, 2 \mathrm{H}), 7.14$ (ddd, $J=7.7,1.9$, $0.8 \mathrm{~Hz}, 2 \mathrm{H}), 7.10(\mathrm{~s}, 2 \mathrm{H}), 7.08(\mathrm{~s}, 1 \mathrm{H}), 6.75(\mathrm{~s}, 2 \mathrm{H}), 5.75(\mathrm{~s}, 1 \mathrm{H})$, 5.25 (s, 2H), 2.23 (s, 6H). Rt: $3.02(254 \mathrm{~nm}) ;(\mathrm{m} / \mathrm{z}): 555.0(\mathrm{M}+1)$.

5-(2,8-Dimethyl-5H-dibenzo[a,d][7]annulen-5-yl)-1-methyl-4-thioxo-3,4-dihydropyrimidin-2(1H)-one (4). Compound 4 was prepared using the procedures for 34 and 6 with 5-(2,8-dimethyl-5Hdibenzo[a,d][7]annulen-5-yl)pyrimidine-2,4(1H,3H)-dione $(0.14 \mathrm{~g}$, $0.42 \mathrm{mmol}$ ). Yield: $0.08 \mathrm{~g}, 0.2 \mathrm{mmol}, 48 \%$ over the two steps (yellow solid). ${ }^{1} \mathrm{H}$ NMR (400 MHz, DMSO) $\delta: 12.53(\mathrm{~s}, 1 \mathrm{H}), 7.47$ (d, $J=$ $7.8 \mathrm{~Hz}, 2 \mathrm{H}), 7.20$ (s, 2H), 7.16 (ddd, $J=7.8,1.9,0.8 \mathrm{~Hz}, 2 \mathrm{H}), 7.01$ (s, 1H), 6.95 (s, 2H), $5.82(\mathrm{~s}, 1 \mathrm{H}), 3.17$ (s, 3H), 2.29 (s, 6H). Rt: 2.87 $(254 \mathrm{~nm}) ;(\mathrm{m} / z): 361.4(\mathrm{M}+1)$

2-((5-(7-Chloro-2-methyl-4H-benzo[5,6]cyclohepta[1,2-d]thiazol4-yl)-2-oxo-4-thioxo-3,4-dihydropyrimidin-1(2H)-yl)methyl)- $\mathrm{N}-(1 \mathrm{H}$ tetrazol-5-yl)thiazole-4-carboxamide (5). A stirred solution of 36 $(65 \mathrm{mg}, 0.120 \mathrm{mmol})$ in $\mathrm{MeOH}(10 \mathrm{~mL})$ was treated with $2 \mathrm{M} \mathrm{NaOH}$ $(0.18 \mathrm{~mL}, 0.36 \mathrm{mmol})$ and heated to reflux for 30 min under $\mathrm{N}_{2}$. Completion was observed by TLC, and the RM was concentrated to one-third the volume, diluted with ethyl acetate $(20 \mathrm{~mL})$, washed with $1 \mathrm{M} \mathrm{HCl}(10 \mathrm{~mL})$ and brine $(10 \mathrm{~mL})$, dried over $\mathrm{MgSO}_{4}$, filtered, and concentrated in vacuo to afford the corresponding acid as an orange solid $(60 \mathrm{mg})$. A stirred solution of the acid $(30 \mathrm{mg}, 0.058 \mathrm{mmol})$ in DMF $(3 \mathrm{~mL})$ was treated with DIPEA $(40 \mu \mathrm{L})$ and 5-aminotetrazole monohydrate $(12 \mathrm{mg}, 0.117 \mathrm{mmol}$ ) followed by PyBroP (41 mg, $0.087 \mathrm{mmol}$ ) and stirred at $\mathrm{rt}$ for $2 \mathrm{~h}$ until completion was observed by LC-MS. The RM was quenched with water (ca. two drops), diluted with ethyl acetate $(20 \mathrm{~mL})$, and partitioned with $1 \mathrm{M} \mathrm{HCl}(10 \mathrm{~mL})$; the organics were extracted with ethyl acetate $(2 \times 10 \mathrm{~mL})$, combined, washed with brine $(10 \mathrm{~mL})$, dried over $\mathrm{MgSO}_{4}$, filtered, and concentrated in vacuo. The DMF was azeotroped with toluene $(3 \times 50 \mathrm{~mL})$ to afford an orange oil. This was purified by being washed with $10 \%$ $\mathrm{MeOH} / \mathrm{DCM}$ before the desired compound was eluted with $10 \%$ $\mathrm{MeOH} / \mathrm{DCM}$ and $1 \%$ acetic acid to afford the title compound, $\mathbf{5}$, as an orange solid (10 mg, $0.017 \mathrm{mmol}, 30 \%)$. LC-MS (ESI+) Rt: $2.79 \mathrm{~min}$ $\left(254 \mathrm{~nm}\right.$, Method 1); $(\mathrm{m} / z)$ : $581.9\left[\mathrm{M}\left({ }^{35} \mathrm{Cl}\right)+\mathrm{H}\right]^{+}$. H.MS-TOF (ESI-) $(m / z):[\mathrm{M}-\mathrm{H}]^{-}$calcd for $\mathrm{C}_{23} \mathrm{H}_{15} \mathrm{ClN}_{9} \mathrm{O}_{2} \mathrm{~S}_{3}, 580.0205$; found, 580.0186. ${ }^{1} \mathrm{H}$ NMR $\left(500 \mathrm{MHz}, \mathrm{DMSO}-d_{6}\right) \delta: 12.90(\mathrm{~s}, 1 \mathrm{H}), 8.50$ $(\mathrm{s}, 1 \mathrm{H}), 7.67(\mathrm{~d}, J=8.4 \mathrm{~Hz}, 1 \mathrm{H}), 7.66(\mathrm{~s}, 1 \mathrm{H}), 7.50(\mathrm{~d}, J=2.3 \mathrm{~Hz}$, $1 \mathrm{H}), 7.42(\mathrm{dd}, J=8.4,2.3 \mathrm{~Hz}, 1 \mathrm{H}), 7.06(\mathrm{~s}, 2 \mathrm{H}), 6.53(\mathrm{~s}, 1 \mathrm{H}), 5.36$ $(\mathrm{d}, J=15.7 \mathrm{~Hz}, 1 \mathrm{H}), 5.30(\mathrm{~d}, J=15.7 \mathrm{~Hz}, 1 \mathrm{H}), 2.59$ (s, 3H). N.B., $-\mathrm{CONH}$ and tetrazole-NH were not observed.

5-(7-Chloro-2-methyl-4H-benzo[5,6]cyclohepta[1,2-d]thiazol-4yl)-1-methyl-4-thioxo-3,4-dihydropyrimidin-2(1H)-one (6). Following general procedure $2,34(60 \mathrm{mg}, 0.161 \mathrm{mmol})$ was converted to the title compound, 6 , which was isolated at $1 \% \mathrm{MeOH} / \mathrm{DCM}$ as a yellow solid (25 mg, $0.065 \mathrm{mmol}, 40 \%)$. LC-MS (ESI+) Rt: $2.85 \mathrm{~min}$ (254 nm, Method 1); $(\mathrm{m} / z): 388.1\left[\mathrm{M}\left({ }^{35} \mathrm{Cl}\right)+\mathrm{H}\right]^{+} .{ }^{1} \mathrm{H}$ NMR $\left(400 \mathrm{MHz}, \mathrm{CDCl}_{3}\right)$ $\delta$ : $9.39(\mathrm{~s}, 1 \mathrm{H}), 7.70(\mathrm{~d}, J=8.3 \mathrm{~Hz}, 1 \mathrm{H}), 7.38(\mathrm{dd}, J=8.3,2.2 \mathrm{~Hz}$, $1 \mathrm{H}), 7.35(\mathrm{~d}, J=2.1 \mathrm{~Hz}, 1 \mathrm{H}), 7.19(\mathrm{~s}, 1 \mathrm{H}), 6.95(\mathrm{~d}, J=11.6 \mathrm{~Hz}, 1 \mathrm{H})$, $6.91(\mathrm{~d}, J=11.6 \mathrm{~Hz}, 1 \mathrm{H}), 6.22(\mathrm{~s}, 1 \mathrm{H}), 3.33(\mathrm{~s}, 3 \mathrm{H}), 2.72(\mathrm{~s}, 3 \mathrm{H})$. ${ }^{13} \mathrm{C}$ NMR $\left(101 \mathrm{MHz} \mathrm{CDCl}_{3}\right) \delta: 188.4,166.9,151.1,147.9,140.4$, $135.7,134.0,132.7,132.5,130.7,129.9,129.3,129.1,120.3,119.2$, 45.9, 37.0, 19.4 .

5-(2-Amino-7-chloro-4H-benzo[5,6]cyclohepta[1,2-d] thiazol-4yl)-1-methyl-4-thioxo-3,4-dihydropyrimidin-2(1H)-one (7). Following general procedure $2,45(45 \mathrm{mg}, 0.121 \mathrm{mmol})$ was converted to the title compound, 7 , and isolated at $2 \% \mathrm{MeOH} / \mathrm{DCM}$ as a yellow solid (4.8 mg, $0.012 \mathrm{mmol}, 10 \%)$. LC-MS (ESI+) Rt: $2.51 \mathrm{~min}(254 \mathrm{~nm}$, Method 1); $(\mathrm{m} / z): 389.0\left[\mathrm{M}\left({ }^{35} \mathrm{Cl}\right)+\mathrm{H}\right]^{+}$.

5-(7-Chloro-2-(phenylamino)-4H-benzo[5,6]cyclohepta[1,2-d]thiazol-4-yl)-1-methyl-4-thioxo-3,4-dihydropyrimidin-2(1H)-one (8). Following general procedure $2,46(45 \mathrm{mg}, 0.100 \mathrm{mmol})$ was converted to the title compound, 8 , which was isolated at $2 \% \mathrm{MeOH} /$ DCM as a yellow solid (39 mg, $0.084 \mathrm{mmol}, 84 \%)$. LC-MS (ESI+) Rt: $3.06 \mathrm{~min}\left(254 \mathrm{~nm} \text {, Method 1); }(\mathrm{m} / z): 465.2\left[\mathrm{M}\left({ }^{35} \mathrm{Cl}\right)+\mathrm{H}\right]\right]^{+} .{ }^{1} \mathrm{H}$ NMR $\left(400 \mathrm{MHz}, \mathrm{DMSO}-d_{6}\right) \delta: 12.73(\mathrm{~s}, 1 \mathrm{H}), 10.38(\mathrm{~s}, 1 \mathrm{H}), 7.94(\mathrm{~s}, 1 \mathrm{H})$, $7.59(\mathrm{dd}, J=8.7,1.1 \mathrm{~Hz}, 2 \mathrm{H}), 7.55(\mathrm{~d}, J=8.7 \mathrm{~Hz}, 1 \mathrm{H}), 7.51(\mathrm{~d}, J=$ $2.3 \mathrm{~Hz}, 1 \mathrm{H}), 7.39$ (dd, $J=8.4,2.3 \mathrm{~Hz}, 1 \mathrm{H}), 7.35(\mathrm{dd}, J=8.6,7.4 \mathrm{~Hz}$, $2 \mathrm{H}), 7.03(\mathrm{~d}, J=11.7 \mathrm{~Hz}, 1 \mathrm{H}), 7.00(\mathrm{~d}, J=11.7 \mathrm{~Hz}, 1 \mathrm{H}), 7.02-6.98$ (m, 1H), $5.74(\mathrm{~s}, 1 \mathrm{H}), 3.39(\mathrm{~s}, 3 \mathrm{H}) .{ }^{13} \mathrm{C}$ NMR $\left(101 \mathrm{MHz}, \mathrm{DMSO}-d_{6}\right)$ $\delta: 189.4,164.0,155.2,148.5,148.4,144.5,141.0,137.6,134.7,130.9$, $129.5,128.9,128.5,128.2,122.4,122.3,118.2,117.8,117.4,44.7,37.3$. 5-(2-(Benzylamino)-7-chloro-4H-benzo[5,6]cyclohepta[1,2-d]thiazol-4-yl)-1-methyl-4-thioxo-3,4-dihydropyrimidin-2(1H)-one (9). Following general procedure 2, $47(30 \mathrm{mg}, 0.065 \mathrm{mmol})$ was converted to the title compound, $\mathbf{9 5}$, which was isolated at $2 \% \mathrm{MeOH} /$ DCM as a yellow solid (25 mg, $0.053 \mathrm{mmol}, 81 \%)$. LC-MS (ESI+) Rt: $3.01 \mathrm{~min}\left(254 \mathrm{~nm}\right.$, Method 1); $(\mathrm{m} / z): 478.9\left[\mathrm{M}\left({ }^{35} \mathrm{Cl}\right)+\mathrm{H}\right]^{+}$. ${ }^{1} \mathrm{H}$ NMR $\left(400 \mathrm{MHz}, \mathrm{DMSO}-d_{6}\right) \delta: 12.65(\mathrm{~s}, 1 \mathrm{H}), 8.42(\mathrm{t}, J=5.8 \mathrm{~Hz}, 1 \mathrm{H}), 7.63$ $(\mathrm{s}, 1 \mathrm{H}), 7.54(\mathrm{~d}, J=8.5 \mathrm{~Hz}, 1 \mathrm{H}), 7.44(\mathrm{~d}, J=2.3 \mathrm{~Hz}, 1 \mathrm{H})$, 
7.34-7.30 (m, 5H), 7.27-7.24 (m, 1H), $6.86(\mathrm{~s}, 2 \mathrm{H}), 5.69(\mathrm{~s}, 1 \mathrm{H})$, $4.48(\mathrm{dd}, J=15.3,5.9 \mathrm{~Hz}, 1 \mathrm{H}), 4.41(\mathrm{dd}, J=15.3,5.6 \mathrm{~Hz}, 1 \mathrm{H}), 3.26$ $(\mathrm{s}, 3 \mathrm{H}) .{ }^{13} \mathrm{C}$ NMR (101 MHz, DMSO- $\left.d_{6}\right) \delta: 189.2,169.5,148.8$, $148.5,143.5,139.0,137.6,134.2,131.8,130.8,129.01,128.84,127.93$, 127.90, 127.6, 126.9, 122.7, 117.5, 117.0, 48.1, 45.1, 37.2.

5-(7-Chloro-2-(phenethylamino)-4H-benzo[5,6]cyclohepta[1,2d]thiazol-4-yl)-1-methyl-4-thioxo-3,4-dihydropyrimidin-2(1H)-one (10). Following general procedure 2, $48(35 \mathrm{mg}, 0.071 \mathrm{mmol})$ was converted to the title compound, 10, at $2 \% \mathrm{MeOH} / \mathrm{DCM}$ as a yellow solid (21 mg, $0.043 \mathrm{mmol}, 60 \%)$. LC-MS (ESI+) Rt: $3.03 \mathrm{~min}(254 \mathrm{~nm}$, Method 1); $(m / z): 492.8\left[\mathrm{M}\left({ }^{35} \mathrm{Cl}\right)+\mathrm{H}\right]^{+} .{ }^{1} \mathrm{H}$ NMR $(400 \mathrm{MHz}$, DMSO- $\left.d_{6}\right) \delta: 12.67(\mathrm{~s}, 1 \mathrm{H}), 8.04(\mathrm{t}, J=5.4 \mathrm{~Hz}, 1 \mathrm{H}), 7.64(\mathrm{~s}, 1 \mathrm{H})$, $7.57(\mathrm{~d}, J=8.6 \mathrm{~Hz}, 1 \mathrm{H}), 7.45(\mathrm{~d}, J=2.3 \mathrm{~Hz}, 1 \mathrm{H}), 7.35(\mathrm{dd}, J=8.4$, $2.3 \mathrm{~Hz}, 1 \mathrm{H}), 7.32-7.24(\mathrm{~m}, 4 \mathrm{H}), 7.22-7.18(\mathrm{~m}, 1 \mathrm{H}), 6.87(\mathrm{~s}, 2 \mathrm{H})$, $5.72(\mathrm{~s}, 1 \mathrm{H}), 3.52-3.45(\mathrm{~m}, 2 \mathrm{H}), 3.27(\mathrm{~s}, 3 \mathrm{H}), 2.87(\mathrm{t}, J=7.3 \mathrm{~Hz}$ $2 \mathrm{H}) .{ }^{13} \mathrm{C}$ NMR $\left(101 \mathrm{MHz}\right.$, DMSO- $\left.d_{6}\right) \delta: 189.2,169.3,148.5,143.5$, $142.4,139.7,137.6,134.1,131.9,130.8,129.14,129.01,128.8,127.9$, $126.73,126.61,122.7,117.6,116.6,46.1,45.2,37.2,35.1$.

5-(7-Chloro-2-(4-methylpiperazin-1-yl)-4H-benzo[5,6]cyclohepta[1,2-d]thiazol-4-yl)-1-methyl-4-thioxo-3,4-dihydropyrimidin-2(1H)one (11). Following general procedure 2, $49(47 \mathrm{mg}, 0.103 \mathrm{mmol})$ was converted to the title compound, 11, which was isolated at $5 \% \mathrm{MeOH} /$ DCM with $0.1 \% 880 \mathrm{NH}_{3}$ as a yellow solid ( $\left.4.5 \mathrm{mg}, 0.0095 \mathrm{mmol}, 9 \%\right)$. LC-MS (ESI+) Rt: $2.28 \mathrm{~min}(254 \mathrm{~nm}$, Method 1); $(\mathrm{m} / \mathrm{z}): 472.1$ $\left.\left[\mathrm{M}\left({ }^{35} \mathrm{Cl}\right)+\mathrm{H}\right]\right]^{+}$. ${ }^{1} \mathrm{H}$ NMR $\left(400 \mathrm{MHz}\right.$, DMSO- $\left.d_{6}\right) \delta: 12.67(\mathrm{~s}, 1 \mathrm{H})$, $7.61(\mathrm{~d}, J=8.5 \mathrm{~Hz}, 1 \mathrm{H}), 7.54(\mathrm{~s}, 1 \mathrm{H}), 7.47(\mathrm{~d}, J=2.3 \mathrm{~Hz}, 1 \mathrm{H}), 7.36$ $(\mathrm{dd}, J=8.4,2.4 \mathrm{~Hz}, 1 \mathrm{H}), 6.94(\mathrm{~d}, J=11.6 \mathrm{~Hz}, 1 \mathrm{H}), 6.91(\mathrm{~d}, J=11.7 \mathrm{~Hz}$, $1 \mathrm{H}), 5.79(\mathrm{~s}, 1 \mathrm{H}), 3.46-3.41(\mathrm{~m}, 4 \mathrm{H}), 3.28(\mathrm{~s}, 3 \mathrm{H}), 2.47-2.42$ (m, 4H), $2.27(\mathrm{~s}, 3 \mathrm{H})$.

5-(7-Chloro-2-morpholino-4H-benzo[5,6]cyclohepta[1,2-d]thiazol-4-yl)-1-methyl-4-thioxo-3,4-dihydropyrimidin-2 $(1 \mathrm{H})$-one (12). Following general procedure 2, $50(44 \mathrm{mg}, 0.100 \mathrm{mmol})$ was converted to the title compound, 12, which was isolated at $2 \%$ $\mathrm{MeOH} / \mathrm{DCM}$ as a yellow solid $(20 \mathrm{mg}, 0.044 \mathrm{mmol}, 44 \%)$. LC-MS (ESI+) Rt: $2.94 \mathrm{~min}$ (254 nm, Method 1); $(\mathrm{m} / \mathrm{z}): 459.2$ $\left[\mathrm{M}\left({ }^{35} \mathrm{Cl}\right)+\mathrm{H}\right]^{+} .{ }^{1} \mathrm{H}$ NMR $\left(400 \mathrm{MHz}\right.$, DMSO- $\left.d_{6}\right) \delta: 12.66(\mathrm{~s}, 1 \mathrm{H})$, $7.59(\mathrm{~d}, J=8.5 \mathrm{~Hz}, 1 \mathrm{H}), 7.56(\mathrm{~s}, 1 \mathrm{H}), 7.46(\mathrm{~d}, J=2.3 \mathrm{~Hz}, 1 \mathrm{H}), 7.35$ (dd, $J=8.4,2.3 \mathrm{~Hz}, 1 \mathrm{H}), 6.95(\mathrm{~d}, J=11.6 \mathrm{~Hz}, 1 \mathrm{H}), 6.91(\mathrm{~d}, J=$ $11.7 \mathrm{~Hz}, 1 \mathrm{H}), 5.77(\mathrm{~s}, 1 \mathrm{H}), 3.69(\mathrm{t}, J=4.9 \mathrm{~Hz}, 4 \mathrm{H}), 3.39(\mathrm{t}, J=4.8 \mathrm{~Hz}$, $4 \mathrm{H}), 3.28(\mathrm{~s}, 3 \mathrm{H}) .{ }^{13} \mathrm{C}$ NMR (101 MHz, DMSO- $\left.d_{6}\right) \delta: 189.2,171.3$, 149.2 , 148.5, 143.3, 137.4, 134.1, 132.2, 131.0, 129.2, 128.0, 127.6, $122.4,118.5,117.3,65.8,48.3,45.4,37.2$.

5-(7-Chloro-2-(piperidin-1-yl)-4H-benzo[5,6]cyclohepta[1,2-d]thiazol-4-yl)-1-methyl-4-thioxo-3,4-dihydropyrimidin-2(1H)-one (13). Following general procedure 2, 51 (44 $\mathrm{mg}, 0.100 \mathrm{mmol}$ ) was converted to the title compound, $\mathbf{1 3}$, which was isolated at $2 \% \mathrm{MeOH} /$ DCM as a yellow solid ( $34 \mathrm{mg}, 0.074 \mathrm{mmol}, 74 \%)$. LC-MS (ESI+) Rt: $3.14 \mathrm{~min}(254 \mathrm{~nm}$, Method 1$) ;(\mathrm{m} / z): 456.8\left[\mathrm{M}\left({ }^{35} \mathrm{Cl}\right)+\mathrm{H}\right]^{+}$. ${ }^{1} \mathrm{H}$ NMR $\left(400 \mathrm{MHz}, \mathrm{DMSO}-d_{6}\right) \delta: 12.65(\mathrm{~s}, 1 \mathrm{H}), 7.60(\mathrm{~d}, J=8.5 \mathrm{~Hz}, 1 \mathrm{H}), 7.51$ $(\mathrm{s}, 1 \mathrm{H}), 7.44(\mathrm{~d}, J=2.3 \mathrm{~Hz}, 1 \mathrm{H}), 7.34(\mathrm{dd}, J=8.4,2.3 \mathrm{~Hz}, 1 \mathrm{H}), 6.90$ $(\mathrm{d}, J=11.6 \mathrm{~Hz}, 1 \mathrm{H}), 6.87(\mathrm{~d}, J=11.7 \mathrm{~Hz}, 1 \mathrm{H}), 5.76(\mathrm{~s}, 1 \mathrm{H}), 3.40$ (s, 4H), $3.27(\mathrm{~s}, 3 \mathrm{H}), 1.57(\mathrm{~s}, 6 \mathrm{H}) \cdot{ }^{13} \mathrm{C}$ NMR (101 MHz, DMSO-d 6 ) $\delta: 189.2,171.0,149.5,148.5,143.1,137.4,133.9,132.4,130.9,129.2$, 127.9, 127.0, 122.4, 117.8, 117.3, 49.2, 45.5, 37.2, 25.1, 23.9.

5-(7-Chloro-2-((2-methoxyethyl)amino )-4H-benzo[5,6]cyclohepta[1,2-d]thiazol-4-yl)-1-methyl-4-thioxo-3,4-dihydropyrimidin-2(1H)-one (14). Following general procedure 2, $52(40 \mathrm{mg}$, $0.093 \mathrm{mmol}$ ) was converted to the title compound, 14, at $2 \% \mathrm{MeOH} /$ DCM as a yellow solid (14 mg, $0.031 \mathrm{mmol}, 34 \%)$. LC-MS (ESI+) Rt: $2.74 \mathrm{~min}(254 \mathrm{~nm}$, Method 1$\left.) ;(\mathrm{m} / z): 446.9\left[\mathrm{M}\left({ }^{35} \mathrm{Cl}\right)+\mathrm{H}\right]\right]^{+} .{ }^{1} \mathrm{H}$ NMR $\left(400 \mathrm{MHz}\right.$, DMSO- $\left.d_{6}\right) \delta: 12.65(\mathrm{~s}, 1 \mathrm{H}), 8.00(\mathrm{t}, J=5.4 \mathrm{~Hz}, 1 \mathrm{H}), 7.63$ $(\mathrm{s}, 1 \mathrm{H}), 7.55(\mathrm{~d}, J=8.5 \mathrm{~Hz}, 1 \mathrm{H}), 7.44(\mathrm{~d}, J=2.3 \mathrm{~Hz}, 1 \mathrm{H}), 7.33(\mathrm{dd}$, $J=8.4,2.3 \mathrm{~Hz}, 1 \mathrm{H}), 6.86(\mathrm{~s}, 2 \mathrm{H}), 5.68(\mathrm{~s}, 1 \mathrm{H}), 3.49-3.46(\mathrm{~m}, 2 \mathrm{H})$, 3.43-3.39 (m, 2H), $3.28(\mathrm{~s}, 3 \mathrm{H}), 3.26(\mathrm{~s}, 3 \mathrm{H}) .{ }^{13} \mathrm{C} \mathrm{NMR}(101 \mathrm{MHz}$, DMSO- $\left.d_{6}\right) \delta: 189.2,169.4,148.8,148.5,143.5,137.6,134.1,130.8$, 129.0, 127.8, 126.8, 122.7, 117.4, 116.7, 70.6, 58.4, 45.2, 44.2, 37.2.

The two enantiomers were isolated by chiral-HPLC: 20 (Rt: $34.46 \mathrm{~min}$, 99\% ee) and 21 (Rt: $43.43 \mathrm{~min}, 78 \%$ ee).

5-(7-Chloro-2-((3-methoxypropyl)amino)-4H-benzo[5,6]cyclohepta[1,2-d] thiazol-4-yl)-1-methyl-4-thioxo-3,4-dihydropyrimidin-2(1H)-one (15). Following general procedure 2, 53
(36 $\mathrm{mg}, 0.080 \mathrm{mmol}$ ) was converted to the title compound, 15, which was isolated at $2 \% \mathrm{MeOH} / \mathrm{DCM}$ as a yellow solid (35 mg, $0.076 \mathrm{mmol}$, 95\%). LC-MS (ESI+) Rt: $2.75 \mathrm{~min}(254 \mathrm{~nm}$, Method 1); $(m / z): 461.0\left[\mathrm{M}\left({ }^{35} \mathrm{Cl}\right)+\mathrm{H}\right]^{+} .{ }^{1} \mathrm{H}$ NMR $\left(400 \mathrm{MHz}, \mathrm{DMSO}-d_{6}\right) \delta: 12.66$ $(\mathrm{s}, 1 \mathrm{H}), 7.94(\mathrm{t}, J=5.5 \mathrm{~Hz}, 1 \mathrm{H}), 7.65(\mathrm{~s}, 1 \mathrm{H}), 7.57(\mathrm{~d}, J=8.5 \mathrm{~Hz}, 1 \mathrm{H})$, $7.45(\mathrm{~d}, J=2.3 \mathrm{~Hz}, 1 \mathrm{H}), 7.35(\mathrm{dd}, J=8.4,2.3 \mathrm{~Hz}, 1 \mathrm{H}), 6.87(\mathrm{~s}, 2 \mathrm{H})$, $5.70(\mathrm{~s}, 1 \mathrm{H}), 3.38(\mathrm{t}, J=6.2 \mathrm{~Hz}, 2 \mathrm{H}), 3.30(\mathrm{~s}, 3 \mathrm{H}), 3.28-3.24$ $(\mathrm{m}, 2 \mathrm{H}), 3.23(\mathrm{~s}, 3 \mathrm{H}), 1.78$ (app.qi, $J=6.6 \mathrm{~Hz}, 2 \mathrm{H}) .{ }^{13} \mathrm{C} \mathrm{NMR}$ $\left(101 \mathrm{MHz}, \mathrm{DMSO}-d_{6}\right) \delta: 189.2,169.5,149.0,148.5,143.4,137.6$, $134.1,130.8,129.0,127.8,126.7,122.7,117.5,116.5,110.0,69.8,58.4$, 45.2, 41.9, 37.2, 29.2 .

5-(7-Chloro-2-((2-ethoxyethyl)amino)-4H-benzo[5,6]cyclohepta[1,2-d]thiazol-4-yl)-1-methyl-4-thioxo-3,4-dihydropyrimidin-2(1H)one (16). Following general procedure $2,54(36 \mathrm{mg}, 0.080 \mathrm{mmol})$ was converted to the title compound, $\mathbf{1 6}$, which was isolated at $2 \% \mathrm{MeOH} /$ DCM as a yellow solid (34 mg, $0.074 \mathrm{mmol}, 92 \%)$. LC-MS (ESI+) Rt: $2.81 \mathrm{~min}\left(254 \mathrm{~nm}\right.$, Method 1); $(\mathrm{m} / z): 461.0\left[\mathrm{M}\left({ }^{35} \mathrm{Cl}\right)+\mathrm{H}\right]^{+}$. ${ }^{1} \mathrm{H}$ NMR $\left(400 \mathrm{MHz}, \mathrm{DMSO}-d_{6}\right) \delta: 12.66(\mathrm{~s}, 1 \mathrm{H}), 8.00(\mathrm{t}, J=5.5 \mathrm{~Hz}, 1 \mathrm{H}), 7.64$ (s, 1H), $7.57(\mathrm{~d}, J=8.5 \mathrm{~Hz}, 1 \mathrm{H}), 7.45(\mathrm{~d}, J=2.3 \mathrm{~Hz}, 1 \mathrm{H}), 7.35$ (dd, $J=8.4,2.3 \mathrm{~Hz}, 1 \mathrm{H}), 6.87(\mathrm{~s}, 2 \mathrm{H}), 5.70(\mathrm{~s}, 1 \mathrm{H}), 3.53-3.50(\mathrm{~m}, 2 \mathrm{H})$, $3.45(\mathrm{q}, J=7.0 \mathrm{~Hz}, 2 \mathrm{H}), 3.43-3.38(\mathrm{~m}, 2 \mathrm{H}), 3.30(\mathrm{~s}, 3 \mathrm{H}), 1.11(\mathrm{t}, J=$ $7.0 \mathrm{~Hz}, 3 \mathrm{H}) .{ }^{13} \mathrm{C}$ NMR $\left(101 \mathrm{MHz}\right.$, DMSO- $\left.d_{6}\right) \delta: 189.3,169.4,148.8$, $148.5,143.5,137.6,134.1,132.0,130.8,129.0,127.8,126.7,122.7$, $117.5,116.7,68.5,65.9,45.2,44.5,37.2,15.6$.

The two enantiomers were isolated by chiral-HPLC: 22 (Rt: $28.32 \mathrm{~min}$, $95 \%$ ee) and 23 (Rt: $36.68 \mathrm{~min}, 96 \%$ ee).

5-(7-Chloro-2-((2-isopropoxyethyl)amino)-4H-benzo[5,6]cyclohepta[1,2-d]thiazol-4-yl)-1-methyl-4-thioxo-3,4-dihydropyrimidin-2(1H)-one (17). Following general procedure 2, $55(37 \mathrm{mg}$, $0.080 \mathrm{mmol}$ ) was converted to the title compound, 17 , which was isolated at $2 \% \mathrm{MeOH} / \mathrm{DCM}$ as a yellow solid $(30 \mathrm{mg}, 0.063 \mathrm{mmol}, 79 \%)$. LC-MS (ESI+) Rt: $2.88 \mathrm{~min}$ (254 nm, Method 1); $(\mathrm{m} / z): 475.1$ $\left[\mathrm{M}\left({ }^{35} \mathrm{Cl}\right)+\mathrm{H}\right]^{+} .{ }^{1} \mathrm{H}$ NMR $\left(400 \mathrm{MHz}\right.$, DMSO- $\left.d_{6}\right) \delta: 12.66(\mathrm{~s}, 1 \mathrm{H}), 7.97$ $(\mathrm{t}, J=5.4 \mathrm{~Hz}, 1 \mathrm{H}), 7.63(\mathrm{~s}, 1 \mathrm{H}), 7.57(\mathrm{~d}, J=8.5 \mathrm{~Hz}, 1 \mathrm{H}), 7.45(\mathrm{~d}, J=$ $2.3 \mathrm{~Hz}, 1 \mathrm{H}), 7.35(\mathrm{dd}, J=8.4,2.3 \mathrm{~Hz}, 1 \mathrm{H}), 6.87(\mathrm{~s}, 2 \mathrm{H}), 5.70(\mathrm{~s}, 1 \mathrm{H})$, 3.57 (sep., $J=6.1 \mathrm{~Hz}, 1 \mathrm{H}), 3.52-3.49(\mathrm{~m}, 2 \mathrm{H}), 3.39-3.37(\mathrm{~m}, 2 \mathrm{H})$, $3.30(\mathrm{~s}, 3 \mathrm{H}), 1.08(\mathrm{~d}, J=6.1 \mathrm{~Hz}, 6 \mathrm{H}) .{ }^{13} \mathrm{C}$ NMR $\left(101 \mathrm{MHz}, \mathrm{DMSO}-d_{6}\right)$ $\delta: 189.2,169.4,148.5,143.5,137.6,134.1,132.0,130.8,129.0,127.9$, 126.7, 122.7, 117.5, 116.7, 71.3, 66.0, 45.2, 44.9, 37.2, 22.51, 22.48.

5-(7-Chloro-2-((2-phenoxyethyl)amino)-4H-benzo[5,6]cyclohepta[1,2-d]thiazol-4-yl)-1-methyl-4-thioxo-3,4-dihydropyrimidin-2(1H)-one (18). Following general procedure 2, $56(39 \mathrm{mg}$, $0.080 \mathrm{mmol}$ ) was converted to the title compound, 18. This was isolated at $2 \% \mathrm{MeOH} / \mathrm{DCM}$, and further purification was achieved by preparative RP-HPLC (Method 3), which isolated the title compound, 18, at a retention time of $18.57 \mathrm{~min}$. This compound was freeze-dried to an orange solid ( $8 \mathrm{mg}, 0.016 \mathrm{mmol}, 20 \%)$. LC-MS (ESI+) Rt: $3.02 \mathrm{~min}(254 \mathrm{~nm}$, Method 1$\left.) ;(\mathrm{m} / z): 509.0\left[\mathrm{M}\left({ }^{35} \mathrm{Cl}\right)+\mathrm{H}\right]\right]^{+} .{ }^{1} \mathrm{H}$ NMR $\left(400 \mathrm{MHz}, \mathrm{DMSO}-d_{6}\right) \delta: 12.66(\mathrm{~s}, 1 \mathrm{H}), 8.20(\mathrm{t}, J=5.3 \mathrm{~Hz}, 1 \mathrm{H}), 7.63$ $(\mathrm{s}, 1 \mathrm{H}), 7.57(\mathrm{~d}, J=8.5 \mathrm{~Hz}, 1 \mathrm{H}), 7.45(\mathrm{~d}, J=2.2 \mathrm{~Hz}, 1 \mathrm{H}), 7.35(\mathrm{dd}$, $J=8.4,2.3 \mathrm{~Hz}, 1 \mathrm{H}), 7.29$ (app.t, $J=8.0 \mathrm{~Hz}, 2 \mathrm{H}), 6.97-6.92(\mathrm{~m}, 3 \mathrm{H})$, $6.88(\mathrm{~s}, 2 \mathrm{H}), 5.72(\mathrm{~s}, 1 \mathrm{H}), 4.13(\mathrm{t}, J=5.4 \mathrm{~Hz}, 2 \mathrm{H}), 3.64$ (app.q, $J=$ $5.4 \mathrm{~Hz}, 2 \mathrm{H}), 3.27(\mathrm{~s}, 3 \mathrm{H})$.

5-(7-Chloro-2-phenyl-4H-benzo[5,6]cyclohepta[1,2-d]thiazol-4yl)-1-methyl-4-thioxo-3,4-dihydropyrimidin-2(1H)-one (19). Following general procedure $2,44(15 \mathrm{mg}, 0.035 \mathrm{mmol})$ was converted to the title compound, 19, which was purified by FC (20-50\% ethyl acetate/ petroleum ether) and isolated at 50\% ethyl acetate/petroleum ether as a yellow solid (12 mg, $0.027 \mathrm{mmol}, 76 \%)$. LC-MS (ESI+) Rt: $3.20 \mathrm{~min}$ $\left(254 \mathrm{~nm}\right.$, Method 1); $(\mathrm{m} / z): 450.1\left[\mathrm{M}\left({ }^{35} \mathrm{Cl}\right)+\mathrm{H}\right]^{+}$. LC-MS (ESI+) Rt: $6.99 \mathrm{~min}\left(254 \mathrm{~nm} \text {, Method 2); }(\mathrm{m} / z): 450.0\left[\mathrm{M}\left({ }^{35} \mathrm{Cl}\right)+\mathrm{H}\right]\right]^{+} .{ }^{1} \mathrm{H}$ NMR $\left(400 \mathrm{MHz}, \mathrm{DMSO}-d_{6}\right) \delta: 12.76(\mathrm{~s}, 1 \mathrm{H}), 7.96-7.94(\mathrm{~m}, 2 \mathrm{H}), 7.88$ $(\mathrm{s}, 1 \mathrm{H}), 7.67(\mathrm{~d}, J=8.4 \mathrm{~Hz}, 1 \mathrm{H}), 7.58(\mathrm{~d}, J=2.1 \mathrm{~Hz}, 1 \mathrm{H}), 7.53-7.49$ $(\mathrm{m}, 3 \mathrm{H}), 7.45(\mathrm{dd}, J=8.4,2.2 \mathrm{~Hz}, 1 \mathrm{H}), 7.27(\mathrm{~d}, J=11.6 \mathrm{~Hz}, 1 \mathrm{H}), 7.22$ $(\mathrm{d}, J=11.8 \mathrm{~Hz}, 1 \mathrm{H}), 6.04(\mathrm{~s}, 1 \mathrm{H}), 3.37(\mathrm{~s}, 3 \mathrm{H}) . .{ }^{13} \mathrm{C} \mathrm{NMR}(101 \mathrm{MHz}$ DMSO-d $\left.d_{6}\right) \delta: 189.3,167.2,152.5,148.5,144.1,137.0,135.4,133.2$, $131.60,131.50,131.36,131.1,129.90,129.79,129.61,129.0,126.5$, 122.0, 117.2, 45.0, 37.2.

2-((5-(7-Chloro-2-((2-methoxyethyl)amino)-4H-benzo[5,6]cyclohepta[1,2-d]thiazol-4-yl)-2-oxo-4-thioxo-3,4-dihydropyrimidin-1(2H)-yl)methyl)-N-(1H-tetrazol-5-yl)thiazole-4-carboxamide 
(24). A stirred solution of $59(140 \mathrm{mg}, 0.23 \mathrm{mmol})$ in $\mathrm{MeOH}(10 \mathrm{~mL})$ was treated with $2 \mathrm{M} \mathrm{NaOH}(0.35 \mathrm{~mL}, 0.69 \mathrm{mmol})$ and heated to reflux for $1 \mathrm{~h}$ under $\mathrm{N}_{2}$. Completion was observed by TLC, and the crude was evaporated directly onto silica, purified by FC, and washed with $5 \% \mathrm{MeOH} / \mathrm{DCM}$ before the desired compound was eluted with $20 \% \mathrm{MeOH} / \mathrm{DCM}$ with $1 \%$ acetic acid to afford a yellow solid. This was dissolved in EA $(20 \mathrm{~mL})$, washed with $1 \mathrm{M} \mathrm{HCl}(10 \mathrm{~mL})$ and brine $(10 \mathrm{~mL})$, dried over $\mathrm{MgSO}_{4}$, filtered, and concentrated in vacuo to afford the corresponding carboxylic acid as an orange solid $(120 \mathrm{mg}$, $0.20 \mathrm{mmol}, 91 \%)$. LC-MS (ESI+) Rt: $2.68 \mathrm{~min}(254 \mathrm{~nm}$, Method 1); $(\mathrm{m} / z): 573.9\left[\mathrm{M}\left({ }^{35} \mathrm{Cl}\right)+\mathrm{H}\right]^{+}$. The acid $(20 \mathrm{mg}, 0.035 \mathrm{mmol})$ in DMF $(1.5 \mathrm{~mL})$ was treated with DIPEA $(50 \mu \mathrm{L})$ and 5 -aminotetrazole monohydrate $(22 \mathrm{mg}, 0.203 \mathrm{mmol}$ ) followed by PyBroP $(24 \mathrm{mg}$, $0.051 \mathrm{mmol}$ ) and stirred at $\mathrm{rt}$ for $2 \mathrm{~h}$ until completion was observed by LC-MS. The RM was quenched with water (ca. two drops), diluted with EA $(20 \mathrm{~mL})$, and partitioned with $1 \mathrm{M} \mathrm{HCl}(10 \mathrm{~mL})$, and the organics were extracted with EA $(2 \times 10 \mathrm{~mL})$, combined, washed with brine $(10 \mathrm{~mL})$, dried over $\mathrm{MgSO}_{4}$, filtered, and concentrated in vacuo. The DMF was azeotroped with toluene $(3 \times 50 \mathrm{~mL})$ to afford an orange oil. This was purified by being washed with $10 \% \mathrm{MeOH} / \mathrm{DCM}$ before the desired compound was eluted with $10 \% \mathrm{MeOH} / \mathrm{DCM}$ with $1 \%$ acetic acid to afford the title compound. Further purification was achieved by preparative RP-HPLC (Method 3), isolating the title compound, 24, at a retention time of $7.37 \mathrm{~min}$. This compound was freeze-dried to an orange solid ( $8 \mathrm{mg}, 0.016 \mathrm{mmol}, 34 \%)$. LC-MS (ESI+) Rt: $2.71 \mathrm{~min}(254 \mathrm{~nm}$, Method 1); $(\mathrm{m} / z)$ : 640.9 $\left[\mathrm{M}\left({ }^{35} \mathrm{Cl}\right)+\mathrm{H}\right]^{+}$. LC-MS (ESI+) Rt: $5.12 \mathrm{~min}(254 \mathrm{~nm}$, Method 2); $(\mathrm{m} / \mathrm{z}): 640.9\left[\mathrm{M}\left({ }^{35} \mathrm{Cl}\right)+\mathrm{H}\right]^{+}$. H.MS-TOF (ESI-) $(\mathrm{m} / \mathrm{z}):[\mathrm{M}-\mathrm{H}]^{-}$ calcd for $\mathrm{C}_{25} \mathrm{H}_{20} \mathrm{ClN}_{10} \mathrm{O}_{3} \mathrm{~S}_{3}, 639.0576$; found, 639.0569. ${ }^{1} \mathrm{H}$ NMR $\left(400 \mathrm{MHz}, \mathrm{DMSO}-d_{6}\right) \delta: 12.89(\mathrm{~s}, 1 \mathrm{H}), 12.30-12.23(\mathrm{~m}, 1 \mathrm{H}), 8.74$ (s, $1 \mathrm{H}), 7.98(\mathrm{t}, J=4.9 \mathrm{~Hz}, 1 \mathrm{H}), 7.76(\mathrm{~s}, 1 \mathrm{H}), 7.57(\mathrm{~d}, J=8.5 \mathrm{~Hz}$, $1 \mathrm{H}), 7.40(\mathrm{~d}, J=2.2 \mathrm{~Hz}, 1 \mathrm{H}), 7.34(\mathrm{dd}, J=8.4,2.3 \mathrm{~Hz}, 1 \mathrm{H}), 6.76(\mathrm{~s}$, $2 \mathrm{H}), 5.67(\mathrm{~s}, 1 \mathrm{H}), 5.45(\mathrm{~d}, J=16.0 \mathrm{~Hz}, 1 \mathrm{H}), 5.30(\mathrm{~d}, J=16.0 \mathrm{~Hz}, 1 \mathrm{H})$, $3.42-3.33(\mathrm{~m}, 4 \mathrm{H}), 3.22(\mathrm{~s}, 3 \mathrm{H}) .{ }^{13} \mathrm{C}$ NMR $\left(126 \mathrm{MHz}, \mathrm{DMSO}-d_{6}\right) \delta$ : $189.8,176.9,169.4,165.9,159.4,148.0,141.9,137.5,133.7,131.2$, $130.8,129.44,129.35,128.9,128.7,127.8,126.7,122.6,117.7,116.7$, 70.6, 58.3, 49.4, 46.3, 44.1.

3-(3-Chlorophenyl)propanal (26). A stirred solution of 3-chloroiodobenzene $(50.00 \mathrm{~g}, 210 \mathrm{mmol})$, allyl alcohol $(21.4 \mathrm{~mL}$, $18.27 \mathrm{~g}, 314 \mathrm{mmol})$, tetrabutylammonium chloride ( $58.26 \mathrm{~g}, 210 \mathrm{mmol})$, and $\mathrm{NaHCO}_{3}(17.61 \mathrm{~g}, 210 \mathrm{mmol})$ dissolved in anhydrous DMF $(150 \mathrm{~mL})$ and cooled in an ice bath under $\mathrm{N}_{2}$ was treated portionwise with $\mathrm{Pd}(\mathrm{OAc})_{2}(1.40 \mathrm{~g}, 6.29 \mathrm{mmol})$ over $30 \mathrm{~min}$. This was then heated to $50{ }^{\circ} \mathrm{C}$ for $18 \mathrm{~h}$, and the consumption of 3-chloroiodobenzene was observed by TLC ( $15 \%$ ethyl acetate/petroleum ether). The reaction was concentrated in vacuo, and the DMF was azeotroped with toluene $(3 \times 200 \mathrm{~mL})$. The resulting black gum was dissolved in $\mathrm{Et}_{2} \mathrm{O}$ $(300 \mathrm{~mL})$ and water $(300 \mathrm{~mL})$ and filtered, and the organics were extracted with $\mathrm{Et}_{2} \mathrm{O}(2 \times 300 \mathrm{~mL})$, combined, washed with brine $(100 \mathrm{~mL})$, dried over $\mathrm{MgSO}_{4}$, and concentrated in vacuo to afford a black oil. Further purification was achieved by $\mathrm{FC}\left(10-40 \% \mathrm{Et}_{2} \mathrm{O} /\right.$ $\mathrm{PE}$ ), to afford the title compound, 27 (32.30 g, $192 \mathrm{mmol}, 92 \%)$. LC-MS (ESI+) Rt: $2.72 \mathrm{~min}(254 \mathrm{~nm}$, Method 1); $(\mathrm{m} / z)$ : not observed. ${ }^{1} \mathrm{H}$ NMR $\left(400 \mathrm{MHz}, \mathrm{CDCl}_{3}\right) \delta: 9.81(\mathrm{t}, J=1.2 \mathrm{~Hz}, 1 \mathrm{H})$, $7.22-7.17(\mathrm{~m}, 3 \mathrm{H}), 7.07(\mathrm{dt}, J=7.1,1.6 \mathrm{~Hz}, 1 \mathrm{H}), 2.93(\mathrm{t}, J=7.4 \mathrm{~Hz}$, $2 \mathrm{H}), 2.80-2.76(\mathrm{~m}, 2 \mathrm{H}) .{ }^{13} \mathrm{C} \mathrm{NMR}\left(101 \mathrm{MHz}^{\left.-\mathrm{CDCl}_{3}\right)} \delta: 200.9\right.$, $142.5,134.3,129.9,128.5,126.6,126.51,44.9,27.7$.

Ethyl 5-(3-Chlorophenethyl)-2-methylthiazole-4-carboxylate (27). A stirred solution of $26(24.00 \mathrm{~g}, 143 \mathrm{mmol})$ and ethyl dichloroacetate $(22.42 \mathrm{~g}, 143 \mathrm{mmol})$ dissolved in anhydrous diethyl ether $(120 \mathrm{~mL})$ and cooled to below $-10{ }^{\circ} \mathrm{C}$ in an ice-salt bath under $\mathrm{N}_{2}$ was treated with a freshly prepared solution of sodium ethoxide $(2.2 \mathrm{M}, 50 \mathrm{~mL}, 110 \mathrm{mmol}$ ) over a $15 \mathrm{~min}$ period, and we ensured that the temperature did not rise above $0{ }^{\circ} \mathrm{C}$. This generated a pale-orange suspension. This was stirred for $45 \mathrm{~min}$ and then warmed to $40{ }^{\circ} \mathrm{C}$ over $30 \mathrm{~min}$. This was then quenched with water $(250 \mathrm{~mL})$, and the organics were extracted with diethyl ether $(3 \times 150 \mathrm{~mL})$. These were combined, washed with brine $(100 \mathrm{~mL})$, dried over $\mathrm{MgSO}_{4}$, filtered, and concentrated in vacuo to afford to afford an orange oil. This was dissolved in ethanol $(80 \mathrm{~mL})$ and added dropwise to a warm solution of thioacetamide $(16.09 \mathrm{~g}, 214 \mathrm{mmol})$ in ethanol $(80 \mathrm{~mL})$ that had been heated to $65^{\circ} \mathrm{C}$ over a $10 \mathrm{~min}$ period. This was heated to reflux for $3 \mathrm{~h}$, generating a red solution with a white precipitate, which was allowed to cool to rt and concentrated in vacuo. The residue was then diluted with diethyl ether $(200 \mathrm{~mL})$ and partitioned with $\mathrm{NaHCO}_{3}$ $(100 \mathrm{~mL})$ and water $(100 \mathrm{~mL})$. The organics where extracted with diethyl ether $(3 \times 200 \mathrm{~mL})$, combined, washed with brine $(100 \mathrm{~mL})$, dried over $\mathrm{MgSO}_{4}$, filtered, and concentrated in vacuo to afford a brown oil. This was purified by FC (10-30\% ethyl acetate/petroleum ether) to afford the title compound, 27 , at $15 \%$ ethyl acetate/ petroleum ether as a yellow solid (13.21 g, $42.7 \mathrm{mmol}, 30 \%)$. LC-MS (ESI+) Rt: $3.04 \mathrm{~min}(254 \mathrm{~nm}$, Method 1); $(\mathrm{m} / z): 310.0$ $\left.\left[\mathrm{M}\left({ }^{35} \mathrm{Cl}\right)+\mathrm{H}\right]\right]^{+1} \mathrm{H}$ NMR $\left(400 \mathrm{MHz}, \mathrm{CDCl}_{3}\right) \delta: 7.21-7.19(\mathrm{~m}, 3 \mathrm{H})$, $7.08-7.06(\mathrm{~m}, 1 \mathrm{H}), 4.42(\mathrm{q}, J=7.1 \mathrm{~Hz}, 2 \mathrm{H}), 3.48(\mathrm{t}, J=7.8 \mathrm{~Hz}, 2 \mathrm{H})$, $2.95(\mathrm{t}, J=7.8 \mathrm{~Hz}, 2 \mathrm{H}), 2.67(\mathrm{~s}, 3 \mathrm{H}), 1.41(\mathrm{t}, J=7.1 \mathrm{~Hz}, 3 \mathrm{H})$. ${ }^{13} \mathrm{C}$ NMR $\left(101 \mathrm{MHz}, \mathrm{CDCl}_{3}\right) \delta: 162.5,162.2,148.4,142.3,140.6$, 134.2, 129.7, 128.6, 126.73, 126.59, 61.2, 37.0, 28.8, 19.3, 14.5.

5-(3-Chlorophenethyl)-2-methylthiazole-4-carboxylic Acid (28). A stirred solution of $27(13.00 \mathrm{~g}, 42.0 \mathrm{mmol})$ in THF $(60 \mathrm{~mL})$ was treated with a solution of $\mathrm{NaOH}(2.52 \mathrm{~g}, 63.0 \mathrm{mmol})$ in water $(60 \mathrm{~mL})$. This was stirred for $24 \mathrm{~h}$ at $\mathrm{rt}$ and treated with $2 \mathrm{M} \mathrm{HCl}(100 \mathrm{~mL})$, and the organics were extracted with ethyl acetate $(3 \times 100 \mathrm{~mL})$, combined, washed with brine $(100 \mathrm{~mL})$, dried over $\mathrm{MgSO}_{4}$, filtered, and concentrated in vacuo to afford the title compound, 28 , as a paleyellow solid (11.68 g, $41.4 \mathrm{mmol}, 99 \%) .{ }^{1} \mathrm{H}$ NMR (400 MHz, $\mathrm{CDCl}_{3}$ ) $\delta$ : 9.38-9.07 (br.s, $1 \mathrm{H}), 7.25-7.19(\mathrm{~m}, 3 \mathrm{H}), 7.10(\mathrm{dt}, J=6.5,1.9 \mathrm{~Hz}$, $1 \mathrm{H}), 3.52(\mathrm{t}, J=7.8 \mathrm{~Hz}, 2 \mathrm{H}), 2.98(\mathrm{t}, J=7.8 \mathrm{~Hz}, 2 \mathrm{H}), 2.69(\mathrm{~s}, 3 \mathrm{H})$. ${ }^{13} \mathrm{C}$ NMR (101 MHz, $\left.\mathrm{CDCl}_{3}\right) \delta: 163.2,149.6,142.0,139.6,134.3$, $129.8,128.7,126.8,126.7,36.8,28.8,18.9$.

7-Chloro-2-methyl-9,10-dihydro-4H-benzo[5,6]cyclohepta[1,2d] thiazol-4-one (29). A stirred solution of $28(11.27 \mathrm{~g}, 40 \mathrm{mmol})$ in DCM $(120 \mathrm{~mL})$ under $\mathrm{N}_{2}$ was treated with oxalyl chloride $(6.00 \mathrm{~mL}$, $70 \mathrm{mmol}$ ) and catalytic DMF (ca. two drops) and stirred for $3 \mathrm{~h}$ at rt. This was then concentrated in vacuo. The residual oxalyl chloride was azeotroped with toluene $(3 \times 50 \mathrm{~mL})$ to afford an orange solid. This was dissolved in DCM $(175 \mathrm{~mL})$, cooled in an ice bath under $\mathrm{N}_{2}$, and treated portionwise with aluminum(III)chloride $(21.33 \mathrm{~g}, 160 \mathrm{mmol})$, generating a black solution. This was allowed to warm to $\mathrm{rt}$ and was stirred for $18 \mathrm{~h}$. This was gradually added to a stirred slurry of ice and $2 \mathrm{M} \mathrm{HCl}(400 \mathrm{~mL})$ and allowed to warm to rt. The organics were extracted with ethyl acetate $(3 \times 400 \mathrm{~mL})$, combined, washed with brine $(300 \mathrm{~mL})$, dried over $\mathrm{MgSO}_{4}$, filtered, and concentrated in vacuo to afford a crude solid. This was purified by FC (20-100\% ethyl acetate/petroleum ether) to afford the title compound, 29 , at $50 \%$ ethyl acetate/petroleum ether as a beige solid (4.16 g, $15.6 \mathrm{mmol}$, $39 \%)$. LC-MS (ESI+) Rt: $2.70 \mathrm{~min}$ (254 nm, Method 1); ( $\mathrm{m} / \mathrm{z}): 264.1$ $\left.\left[\mathrm{M}\left({ }^{35} \mathrm{Cl}\right)+\mathrm{H}\right]\right]^{+1} \mathrm{H}$ NMR $\left(400 \mathrm{MHz}, \mathrm{CDCl}_{3}\right) \delta: 7.77(\mathrm{~d}, J=8.4 \mathrm{~Hz}$, $1 \mathrm{H}), 7.54(\mathrm{~d}, J=2.1 \mathrm{~Hz}, 1 \mathrm{H}), 7.48(\mathrm{dd}, J=8.4,2.2 \mathrm{~Hz}, 1 \mathrm{H}), 3.28-$ $3.25(\mathrm{~m}, 2 \mathrm{H}), 3.21-3.18(\mathrm{~m}, 2 \mathrm{H}), 2.63(\mathrm{~s}, 3 \mathrm{H})$.

5-Bromo-2,4-di-tert-butoxypyrimidine (31). To a stirred solution of 5-bromo-2,4-dichloropyrimidine $(5.00 \mathrm{~g}, 22.00 \mathrm{mmol})$ in anhydrous THF $\left(70 \mathrm{~mL}\right.$ ) cooled in an ice bath under $\mathrm{N}_{2}$, a suspension of sodium tert-butoxide $(6.35 \mathrm{~g}, 66.0 \mathrm{mmol})$ in anhydrous THF $(50 \mathrm{~mL})$ was added dropwise via a dropping funnel over a $30 \mathrm{~min}$ period. This was allowed to warm to rt and was stirred for a further $18 \mathrm{~h}$, generating a dark-brown solution. The $\mathrm{RM}$ was then quenched with $\mathrm{NH}_{4} \mathrm{Cl}(\mathrm{aq})$ $(10 \mathrm{~mL})$ and diluted with water $(100 \mathrm{~mL})$, and the organics were extracted with ethyl acetate $(3 \times 75 \mathrm{~mL})$, combined, washed with brine $(50 \mathrm{~mL})$, dried over $\mathrm{MgSO}_{4}$, filtered, and concentrated in vacuo to afford a black oil. Further purification was achieved by FC $(2.5 \%$ diethyl ether/petroleum ether with $0.1 \% \mathrm{Et}_{3} \mathrm{~N}$ ) to afford the title compound, 31, as a clear oil that crystallized to a white solid on standing (4.08 g, $13.50 \mathrm{mmol}, 61 \%)$. LC-MS (ESI+) Rt: $3.32 \mathrm{~min}(254 \mathrm{~nm}$, Method 1); $(\mathrm{m} / z): 305.2\left[\mathrm{M}\left({ }^{81} \mathrm{Br}\right)+\mathrm{H}\right]^{+}, 303.2\left[\mathrm{M}\left({ }^{79} \mathrm{Br}\right)+\mathrm{H}\right]^{+}$. ${ }^{1} \mathrm{H}$ NMR $\left(400 \mathrm{MHz}, \mathrm{CDCl}_{3}\right) \delta: 8.24(\mathrm{~s}, 1 \mathrm{H}), 1.64(\mathrm{~s}, 9 \mathrm{H}), 1.59$ $(\mathrm{s}, 9 \mathrm{H}) .{ }^{13} \mathrm{C} \mathrm{NMR}\left(101 \mathrm{MHz}, \mathrm{CDCl}_{3}\right) \delta: 165.7,163.0,159.0,99.6$, 83.3, 80.8, 28.3, 28.3.

7-Chloro-4-(2,4-di-tert-butoxypyrimidin-5-yl)-2-methyl-9,10-dihydro-4H-benzo $[5,6]$ cyclohepta $[1,2-d]$ thiazol-4-ol (32). A stirred solution of $31(3.03 \mathrm{~g}, 10.00 \mathrm{mmol})$ in unstabilized, anhydrous 
THF ( $80 \mathrm{~mL}$ ) under $\mathrm{N}_{2}$ was cooled to $-78{ }^{\circ} \mathrm{C}$, treated dropwise with a solution of $n$-butyllithium $(2.46 \mathrm{M}$ in hexane, $4.1 \mathrm{~mL}, 10.50 \mathrm{mmol}$ ), and stirred for $30 \mathrm{~min}$, generating a dark-orange solution. This was then treated dropwise with a solution of $29(2.64 \mathrm{~g}, 10.00 \mathrm{mmol})$ that had been dissolved in unstabilized, anhydrous THF $(20 \mathrm{~mL})$; stirred at $-78{ }^{\circ} \mathrm{C}$ for $15 \mathrm{~min}$; allowed to warm to $\mathrm{rt}$; and stirred again for $1 \mathrm{~h}$. The $\mathrm{RM}$ was then quenched with $\mathrm{NH}_{4} \mathrm{Cl}(50 \mathrm{~mL})$ and diluted with water $(50 \mathrm{~mL})$, and the organics were extracted with ethyl acetate $(3 \times$ $100 \mathrm{~mL})$. These were combined, washed with brine $(100 \mathrm{~mL})$, dried over $\mathrm{MgSO}_{4}$, filtered, and concentrated in vacuo to afford an orange oil. Further purification was achieved by FC (5-20\% ethyl acetate/ petroleum ether) affording the title compound, 32, at $15 \%$ ethyl acetate/petroleum ether as a clear oil that foamed and crystallized to a white solid under high vacuum $(3.04 \mathrm{~g}, 6.23 \mathrm{mmol}, 65 \%)$. LC-MS (ESI+) Rt: $3.36 \min \left(254 \mathrm{~nm}\right.$, Method 1); $(\mathrm{m} / z): 488.2\left[\mathrm{M}\left({ }^{35} \mathrm{Cl}\right)+\mathrm{H}\right]^{+} .{ }^{1} \mathrm{H}$ NMR (400 MHz, DMSO- $\left.d_{6}\right) \delta: 7.88(\mathrm{~s}, 1 \mathrm{H}), 7.38(\mathrm{~d}, J=8.5 \mathrm{~Hz}, 1 \mathrm{H})$, $7.33(\mathrm{~d}, J=2.3 \mathrm{~Hz}, 1 \mathrm{H}), 7.24(\mathrm{dd}, J=8.5,2.4 \mathrm{~Hz}, 1 \mathrm{H}), 6.13$ (s, $1 \mathrm{H}), 3.25-3.18(\mathrm{~m}, 1 \mathrm{H}), 2.97-2.94(\mathrm{~m}, 2 \mathrm{H}), 2.87-2.80(\mathrm{~m}, 1 \mathrm{H})$, $2.53(\mathrm{~s}, 3 \mathrm{H}), 1.56(\mathrm{~s}, 9 \mathrm{H}), 1.18(\mathrm{~s}, 9 \mathrm{H}) .{ }^{13} \mathrm{C}$ NMR $\left(101 \mathrm{MHz}, \mathrm{DMSO}-d_{6}\right)$ $\delta: 167.0,163.2,160.2,156.1,151.7,143.3,141.4,131.84,131.67,129.37$, 129.24, 125.8, 122.1, 81.2, 80.0, 75.3, 31.8, 28.6, 28.1, 26.9, 19.0.

5-(7-Chloro-2-methyl-4H-benzo[5,6]cyclohepta[1,2-d]thiazol-4yl)pyrimidine-2,4(1H,3H)-dione (33). A stirred solution of 32 $(1.54 \mathrm{mmol}, 0.750 \mathrm{~g})$ in trifluoroacetic acid $(20 \mathrm{~mL})$ was heated to reflux under $\mathrm{N}_{2}$ for $72 \mathrm{~h}$, generating a black solution. This was concentrated in vacuo to give a dark-red gum, which was triturated with $\mathrm{Et}_{2} \mathrm{O}(3 \times 10 \mathrm{~mL})$ to give an orange solid $(454 \mathrm{mg})$. This was purified by FC $\left(2-10 \% \mathrm{MeOH} / \mathrm{DCM}\right.$ with $\left.0.1 \% 880 \mathrm{NH}_{3}\right)$ to afford the title compound, 33 , at $4 \% \mathrm{MeOH} / \mathrm{DCM}$ with $0.1 \% 880 \mathrm{NH}_{3}$ as a pink solid (200 mg, $0.56 \mathrm{mmol}, 36 \%)$. LC-MS (ESI+) Rt: $2.80 \mathrm{~min}$ (254 nm, Method 1); $(\mathrm{m} / z): 358.1\left[\mathrm{M}\left({ }^{35} \mathrm{Cl}\right)+\mathrm{H}\right]^{+} .{ }^{1} \mathrm{H} \quad \mathrm{NMR}$ $\left(400 \mathrm{MHz}, \mathrm{DMSO}-d_{6}\right) \delta: 11.07(\mathrm{~s}, 1 \mathrm{H}), 10.57(\mathrm{~d}, J=6.1 \mathrm{~Hz}, 1 \mathrm{H})$, $7.53(\mathrm{~d}, J=8.3 \mathrm{~Hz}, 1 \mathrm{H}), 7.53(\mathrm{~d}, J=2.2 \mathrm{~Hz}, 1 \mathrm{H}), 7.45(\mathrm{dd}, J=8.3$, $2.3 \mathrm{~Hz}, 1 \mathrm{H}), 7.08(\mathrm{~d}, J=11.7 \mathrm{~Hz}, 1 \mathrm{H}), 7.01(\mathrm{~d}, J=11.7 \mathrm{~Hz}, 1 \mathrm{H}), 6.66$ $(\mathrm{d}, J=5.5 \mathrm{~Hz}, 1 \mathrm{H}), 5.50(\mathrm{~s}, 1 \mathrm{H}), 2.64(\mathrm{~s}, 3 \mathrm{H})$.

5-(7-Chloro-2-methyl-4H-benzo[5,6]cyclohepta[1,2-d]thiazol-4yl)-1-methylpyrimidine-2,4(1H,3H)-dione (34). A stirred suspension of $33(100 \mathrm{mg}, 0.28 \mathrm{mmol})$ in DCM $(15 \mathrm{~mL})$ under $\mathrm{N}_{2}$ was treated with $\mathrm{N}, \mathrm{O}$-bis(trimethylsilyl)trifluoroacetamide $(0.3 \mathrm{~mL}, 287 \mathrm{mg}$, $1.18 \mathrm{mmol}$ ) and heated to reflux for $18 \mathrm{~h}$. This was cooled, treated with iodomethane $(1.5 \mathrm{~mL})$, and heated to $50{ }^{\circ} \mathrm{C}$ for $24 \mathrm{~h}$ until completion was observed by LC-MS. This was concentrated in vacuo to afford an oil, which was dissolved in $\mathrm{MeOH}$ and reconcentrated in vacuo to afford a solid. This was purified by FC (1-4\% MeOH/DCM with $0.1 \% 880 \mathrm{NH}_{3}$ ) to afford the title compound, 34 , at $2 \% \mathrm{MeOH} /$ DCM with $0.1 \% 880 \mathrm{NH}_{3}(64 \mathrm{mg}, 0.17 \mathrm{mmol}, 61 \%)$. LC-MS (ESI+) Rt: $2.64 \min \left(254 \mathrm{~nm}\right.$, Method 1); $(\mathrm{m} / z): 372.0\left[\mathrm{M}\left({ }^{35} \mathrm{Cl}\right)+\mathrm{H}\right]^{+} .{ }^{1} \mathrm{H}$ NMR (400 MHz, DMSO- $\left.d_{6}\right) \delta: 11.26(\mathrm{~s}, 1 \mathrm{H}), 7.53(\mathrm{~d}, J=8.4 \mathrm{~Hz}$, $1 \mathrm{H}), 7.51(\mathrm{~d}, J=2.2 \mathrm{~Hz}, 1 \mathrm{H}), 7.44(\mathrm{dd}, J=8.3,2.3 \mathrm{~Hz}, 1 \mathrm{H}), 7.07(\mathrm{~d}, J=$ $11.7 \mathrm{~Hz}, 1 \mathrm{H}), 7.01(\mathrm{~d}, J=11.7 \mathrm{~Hz}, 1 \mathrm{H}), 6.90(\mathrm{~s}, 1 \mathrm{H}), 5.52(\mathrm{~s}, 1 \mathrm{H})$, $3.15(\mathrm{~s}, 3 \mathrm{H}), 2.65(\mathrm{~s}, 3 \mathrm{H})$.

Ethyl 2-((5-(7-Chloro-2-methyl-4H-benzo[5,6]cyclohepta[1,2-d]thiazol-4-yl)-2,4-dioxo-3,4-dihydropyrimidin-1(2H)-yl)methyl)thiazole-4-carboxylate (35). A stirred suspension of $33(160 \mathrm{mg}$, $0.28 \mathrm{mmol})$ in 1,2-dichloroethane $(10 \mathrm{~mL})$ was treated with $\mathrm{N}, \mathrm{O}$ bis(trimethylsilyl)trifluoroacetamide $(0.3 \mathrm{~mL}, 1.18 \mathrm{mmol})$ and heated to reflux for $18 \mathrm{~h}$ under $\mathrm{N}_{2}$. This was then treated with ethyl 5-(bromomethyl)thiophene-3-carboxylate (104 mg, $0.42 \mathrm{mmol}$ ), dissolved in 1,2-dichloroethane $(5 \mathrm{~mL})$, and refluxed for $48 \mathrm{~h}$. This was concentrated in vacuo to a gum, treated with $\mathrm{MeOH}(20 \mathrm{~mL})$, and then reconcentrated in vacuo to a solid. This was purified by chromatography on a silica gel $(1-5 \% \mathrm{MeOH} / \mathrm{DCM})$ to afford the title compound, 35, at $3 \% \mathrm{MeOH} / \mathrm{DCM}(84 \mathrm{mg}, 0.16 \mathrm{mmol}, 57 \%)$. LC-MS (ESI+) Rt: $2.82 \mathrm{~min}(254 \mathrm{~nm}$, Method 1); $(\mathrm{m} / z): 526.9$ $\left[\mathrm{M}\left({ }^{35} \mathrm{Cl}\right)+\mathrm{H}\right]^{+1} \mathrm{H}$ NMR $\left(400 \mathrm{MHz}, \mathrm{CDCl}_{3}\right) \delta: 8.28-8.25$ (br.s, $\left.1 \mathrm{H}\right)$, $8.22(\mathrm{~s}, 1 \mathrm{H}), 7.56(\mathrm{~d}, J=8.3 \mathrm{~Hz}, 1 \mathrm{H}), 7.38(\mathrm{dd}, J=8.3,2.2 \mathrm{~Hz}, 1 \mathrm{H})$, $7.29(\mathrm{~d}, J=2.3 \mathrm{~Hz}, 1 \mathrm{H}), 6.87(\mathrm{~s}, 1 \mathrm{H}), 6.84(\mathrm{~d}, J=11.6 \mathrm{~Hz}, 1 \mathrm{H}), 6.75$ $(\mathrm{d}, J=11.7 \mathrm{~Hz}, 1 \mathrm{H}), 5.79(\mathrm{~s}, 1 \mathrm{H}), 5.21(\mathrm{~d}, J=15.2 \mathrm{~Hz}, 1 \mathrm{H}), 4.98$ $(\mathrm{d}, J=15.2 \mathrm{~Hz}, 1 \mathrm{H}), 4.51(\mathrm{q}, J=7.1 \mathrm{~Hz}, 2 \mathrm{H}), 2.71(\mathrm{~s}, 3 \mathrm{H}), 1.48(\mathrm{t}, J=$ $7.1 \mathrm{~Hz}, 3 \mathrm{H})$.
Ethyl 2-((5-(7-Chloro-2-methyl-4H-benzo[5,6]cyclohepta[1,2-d]thiazol-4-yl)-2-oxo-4-thioxo-3,4-dihydropyrimidin-1(2H)-yl)methyl)thiazole-4-carboxylate (36). Following general procedure 2, 35 ( $80 \mathrm{mg}, 0.151 \mathrm{mmol}$ ) was converted to the title compound, 36 , at $2 \%$ $\mathrm{MeOH} / \mathrm{DCM}$ as a yellow solid (68 mg, $0.125 \mathrm{mmol}, 89 \%)$. LC-MS (ESI+) Rt: $3.01 \mathrm{~min}$ (254 nm, Method 1); $(\mathrm{m} / z): 542.8$ $\left[\mathrm{M}\left({ }^{35} \mathrm{Cl}\right)+\mathrm{H}\right]{ }^{+1} \mathrm{H}$ NMR $\left(400 \mathrm{MHz}, \mathrm{CDCl}_{3}\right) \delta: 9.63(\mathrm{~s}, 1 \mathrm{H}), 8.24$ $(\mathrm{s}, 1 \mathrm{H}), 7.69(\mathrm{~d}, J=8.3 \mathrm{~Hz}, 1 \mathrm{H}), 7.36(\mathrm{dd}, J=8.3,2.1 \mathrm{~Hz}), 7.29(\mathrm{~d}, J=$ $2.2 \mathrm{~Hz}, 1 \mathrm{H}), 7.25(\mathrm{~s}, 1 \mathrm{H}), 6.91(\mathrm{~d}, J=11.6 \mathrm{~Hz}, 1 \mathrm{H}), 6.84(\mathrm{~d}, J=11.5 \mathrm{~Hz}$, $1 \mathrm{H}), 6.20(\mathrm{~s}, 1 \mathrm{H}), 5.26(\mathrm{~d}, J=15.1 \mathrm{~Hz}, 1 \mathrm{H}), 5.03(\mathrm{~d}, J=15.0 \mathrm{~Hz}, 1 \mathrm{H})$, $4.52(\mathrm{q}, J=7.1 \mathrm{~Hz}, 2 \mathrm{H}), 2.71(\mathrm{~s}, 3 \mathrm{H}), 1.49(\mathrm{t}, J=7.1 \mathrm{~Hz}, 3 \mathrm{H})$.

Ethyl 2-Amino-5-(3-chlorophenethyl)thiazole-4-carboxylate (37). A stirred solution of $26(18.50 \mathrm{~g}, 110 \mathrm{mmol})$ and ethyl dichloroacetate $(17.29 \mathrm{~g}, 110 \mathrm{mmol})$, dissolved in anhydrous diethyl ether $(90 \mathrm{~mL})$ and cooled to below $-10{ }^{\circ} \mathrm{C}$ in an ice-salt bath under $\mathrm{N}_{2}$, was treated with a freshly prepared solution of sodium ethoxide $(2.2 \mathrm{M}, 50 \mathrm{~mL}$, $110 \mathrm{mmol}$ ) over a $15 \mathrm{~min}$ period, and we ensured that the temperature did not rise above $0{ }^{\circ} \mathrm{C}$. This generated a pale-orange suspension, which was stirred for $45 \mathrm{~min}$ and warmed to $40{ }^{\circ} \mathrm{C}$ over $30 \mathrm{~min}$. This was then quenched with water $(250 \mathrm{~mL})$, and the organics were extracted with diethyl ether $(3 \times 150 \mathrm{~mL})$. These were combined, washed with brine $(100 \mathrm{~mL})$, dried over $\mathrm{MgSO}_{4}$, filtered, and concentrated in vacuo to afford to afford an orange oil. This was dissolved in ethanol $(60 \mathrm{~mL})$ and added dropwise to a warm solution of thiourea (12.56 g, $165 \mathrm{mmol})$ in ethanol $(60 \mathrm{~mL})$ that had been heated to $65{ }^{\circ} \mathrm{C}$ over a $10 \mathrm{~min}$ period. This was heated to reflux for $2.5 \mathrm{~h}$, generating a purplered solution and a white precipitate, which was then allowed to cool to rt and concentrated in vacuo. The residue was then diluted with diethyl ether $(200 \mathrm{~mL})$ and partitioned with $\mathrm{NaHCO}_{3}(100 \mathrm{~mL})$ and water $(100 \mathrm{~mL})$. The organics were extracted with diethyl ether $(3 \times$ $200 \mathrm{~mL})$, combined, washed with brine $(100 \mathrm{~mL})$, dried over $\mathrm{MgSO}_{4}$, filtered, and concentrated in vacuo to afford a brown oil. This was taken up in a diethyl ether $(100 \mathrm{~mL})$, filtered through a plug of silica, and washed with diethyl ether $(3 \times 200 \mathrm{~mL})$. The filtrate was concentrated to afford an orange solid, which was triturated with diethyl ether/hexane to give the title compound, 37, as a yellow solid (15.70 g, $50.5 \mathrm{mmol}, 46 \%)$. LC-MS (ESI+) Rt: $2.81 \mathrm{~min}(254 \mathrm{~nm}$, Method 1); $(m / z): 311.3\left[\mathrm{M}\left({ }^{35} \mathrm{Cl}\right)+\mathrm{H}\right]^{+} .{ }^{1} \mathrm{H}$ NMR $\left(400 \mathrm{MHz}, \mathrm{CDCl}_{3}\right) \delta: 7.21-$ $7.19(\mathrm{~m}, 3 \mathrm{H}), 7.09-7.07(\mathrm{~m}, 1 \mathrm{H}), 5.09(\mathrm{~s}, 2 \mathrm{H}), 4.36(\mathrm{q}, J=7.1 \mathrm{~Hz}$, $2 \mathrm{H}), 3.37(\mathrm{t}, J=7.8 \mathrm{~Hz}, 2 \mathrm{H}), 2.91(\mathrm{t}, J=7.8 \mathrm{~Hz}, 2 \mathrm{H}), 1.39(\mathrm{t}, J=$ $7.1 \mathrm{~Hz}, 3 \mathrm{H}) .{ }^{13} \mathrm{C} \mathrm{NMR}\left(101 \mathrm{MHz}, \mathrm{CDCl}_{3}\right) \delta: 163.4,162.3,142.6$, 139.5, 136.9, 134.3, 129.8, 128.8, 126.9, 126.6, 61.1, 37.1, 28.8, 14.6.

Ethyl 2-Chloro-5-(3-chlorophenethyl)thiazole-4-carboxylate (38). A stirred solution of $37(15.00 \mathrm{~g}, 48.26 \mathrm{mmol})$ in degassed, anhydrous acetonitrile $(240 \mathrm{~mL})$ under $\mathrm{N}_{2}$ was treated with copper(II)chloride $(12.92 \mathrm{~g}, 96.25 \mathrm{mmol})$ and then treated dropwise, via a syringe pump, with tert-butyl nitrite $(11.50 \mathrm{~mL}, 96.25 \mathrm{mmol})$ over a $15 \mathrm{~min}$ period at rt. We ensured the temperature did not rise above $25^{\circ} \mathrm{C}$. This was stirred for a further $2 \mathrm{~h}$ at rt, quenched with $2 \mathrm{M} \mathrm{HCl}(250 \mathrm{~mL})$, and stirred for $10 \mathrm{~min}$, and then the organics were extracted with ethyl acetate $(3 \times 250 \mathrm{~mL})$. These were combined, washed with brine $(200 \mathrm{~mL})$, dried over $\mathrm{MgSO}_{4}$, filtered, and concentrated in vacuo to afford an orange oil. Further purification was achieved by FC (10\% ethyl acetate/petroleum ether) to afford the title compound, 38, as an orange oil (10.41 g. $31.53 \mathrm{mmol}, 65 \%)$. LC-MS (ESI+) Rt: $3.18 \mathrm{~min}$ $\left(254 \mathrm{~nm}\right.$, Method 1); $(\mathrm{m} / z): 330.0\left[\mathrm{M}\left({ }^{35} \mathrm{Cl}\right)+\mathrm{H}\right]^{+} .{ }^{1} \mathrm{H}$ NMR $(400$ $\left.\mathrm{MHz}, \mathrm{CDCl}_{3}\right) \delta: 7.21-7.18(\mathrm{~m}, 3 \mathrm{H}), 7.05(\mathrm{dt}, J=6.0,2.1 \mathrm{~Hz}, 1 \mathrm{H})$, $4.40(\mathrm{q}, J=7.1 \mathrm{~Hz}, 2 \mathrm{H}), 3.48(\mathrm{t}, J=7.7 \mathrm{~Hz}, 2 \mathrm{H}), 2.95(\mathrm{t}, J=7.7 \mathrm{~Hz}$, $2 \mathrm{H}), 1.40(\mathrm{t}, J=7.1 \mathrm{~Hz}, 3 \mathrm{H}) .{ }^{13} \mathrm{C} \mathrm{NMR}\left(101 \mathrm{MHz}, \mathrm{CDCl}_{3}\right) \delta: 161.2$, $150.5,148.3,141.7,139.8,134.4,129.9,128.6,126.9,126.7,61.6,36.6$, $28.9,14.4$

2-Chloro-5-(3-chlorophenethyl)thiazole-4-carboxylic Acid (39). A stirred solution of $38(10.00 \mathrm{~g}, 30.3 \mathrm{mmol})$ in THF $(40 \mathrm{~mL})$ was treated with a solution of $\mathrm{NaOH}(1.817 \mathrm{~g}, 45.4 \mathrm{mmol})$ in water $(40 \mathrm{~mL})$. This was stirred for $24 \mathrm{~h}$ at $\mathrm{rt}$ and treated with $2 \mathrm{M} \mathrm{HCl}$ $(100 \mathrm{~mL})$. The organics were extracted with ethyl acetate $(3 \times 100 \mathrm{~mL})$, combined, washed with brine $(50 \mathrm{~mL})$, dried over $\mathrm{MgSO}_{4}$, filtered, and concentrated in vacuo to afford the title compound, 39 , as a paleyellow solid (9.15 g, $30.3 \mathrm{mmol}, 100 \%$.). LC-MS (ESI+) Rt: $2.88 \mathrm{~min}$ $\left(254 \mathrm{~nm}\right.$, Method 1); $(\mathrm{m} / \mathrm{z}): 301.9\left[\mathrm{M}\left({ }^{35} \mathrm{Cl}\right)+\mathrm{H}\right]^{+} .{ }^{1} \mathrm{H} \quad \mathrm{NMR}$ 
$\left(400 \mathrm{MHz}, \mathrm{CDCl}_{3}\right) \delta: 7.24-7.20(\mathrm{~m}, 3 \mathrm{H}), 7.09-7.06(\mathrm{~m}, 1 \mathrm{H}), 3.53$ $(\mathrm{t}, J=7.7 \mathrm{~Hz}, 2 \mathrm{H}), 2.98(\mathrm{t}, J=7.7 \mathrm{~Hz}, 2 \mathrm{H}) .{ }^{13} \mathrm{C} \mathrm{NMR}(101 \mathrm{MHz}$, $\left.\mathrm{CDCl}_{3}\right) \delta: 162.4,151.9,148.6,141.4,138.8,134.5,130.0,128.7,127.0$, 126.7, 36.4, 28.9.

2,7-Dichloro-9,10-dihydro-4H-benzo[5,6]cyclohepta[1,2-d]thiazol-4-one (40). A stirred solution of $39(7.00 \mathrm{~g}, 23.17 \mathrm{mmol})$ in DCM $(70 \mathrm{~mL})$ under $\mathrm{N}_{2}$ was treated with oxalyl chloride $(3.47 \mathrm{~mL}$, $40.54 \mathrm{mmol}$ ) and catalytic DMF (ca. two drops) and stirred for $3 \mathrm{~h}$ at rt. This was then concentrated in vacuo. The residual oxalyl chloride was azeotroped with toluene $(3 \times 50 \mathrm{~mL})$ to afford an orange solid. The solid was dissolved in DCM $(100 \mathrm{~mL})$, cooled in an ice bath under $\mathrm{N}_{2}$, and treated portionwise with aluminum(III)chloride (12.36 g, $92.68 \mathrm{mmol})$, generating a black solution. This was allowed to warm to rt and was stirred for $18 \mathrm{~h}$. This was gradually added to a stirred slurry of ice and $2 \mathrm{M} \mathrm{HCl}(250 \mathrm{~mL})$ and allowed to warm to rt. The organics were extracted with ethyl acetate $(3 \times 200 \mathrm{~mL})$, combined, washed with brine $(100 \mathrm{~mL})$, dried over $\mathrm{MgSO}_{4}$, filtered, and concentrated in vacuo to afford an orange solid. This was triturated with diethyl ether/hexane to afford the title compound, 40 , as a beige solid (5.48 g, $19.28 \mathrm{mmol}, 83 \%)$. LC-MS (ESI+) Rt: $2.88 \mathrm{~min}(254 \mathrm{~nm}$, Method 1); $(\mathrm{m} / z): 284.0\left[\mathrm{M}\left({ }^{35} \mathrm{Cl}\right)+\mathrm{H}\right]^{+} .{ }^{1} \mathrm{H}$ NMR $\left(400 \mathrm{MHz}\right.$, DMSO- $\left.d_{6}\right) \delta: 7.78$ $(\mathrm{d}, J=8.4 \mathrm{~Hz}, 1 \mathrm{H}), 7.55(\mathrm{~d}, J=2.1 \mathrm{~Hz}, 1 \mathrm{H}), 7.48(\mathrm{dd}, J=8.4,2.2 \mathrm{~Hz}$, $1 \mathrm{H}), 3.29-3.26(\mathrm{~m}, 2 \mathrm{H}), 3.24-3.21(\mathrm{~m}, 2 \mathrm{H}) .{ }^{13} \mathrm{C}$ NMR $(101 \mathrm{MHz}$, DMSO- $\left.d_{6}\right) \delta: 183.5,153.2,148.6,147.9,142.1,137.7,137.0,132.6$, 129.9, 127.7, 33.5, 26.7

2,7-Dichloro-4-(2,4-di-tert-butoxypyrimidin-5-yl)-9,10-dihydro4H-benzo[5,6]cyclohepta[1,2-d]thiazol-4-ol (41). A stirred solution of 31 (1.516 g, $5.00 \mathrm{mmol})$ in unstabilized, anhydrous THF $(40 \mathrm{~mL})$ under $\mathrm{N}_{2}$ was cooled to $-78{ }^{\circ} \mathrm{C}$, treated dropwise with a solution of $n$-butyllithium ( $2.25 \mathrm{M}$ in hexane, $2.33 \mathrm{~mL}, 5.25 \mathrm{mmol})$, and stirred for $30 \mathrm{~min}$, generating a dark-orange solution. This was then treated dropwise with a solution of $82(1.421 \mathrm{~g}, 5.00 \mathrm{mmol})$ that had been dissolved in unstabilized, anhydrous THF $(10 \mathrm{~mL})$; stirred at $-78{ }^{\circ} \mathrm{C}$ for $15 \mathrm{~min}$; allowed to warm to rt; and stirred for $1 \mathrm{~h}$. The RM was then quenched with $\mathrm{NH}_{4} \mathrm{Cl}(\mathrm{aq})(20 \mathrm{~mL})$ and diluted with water $(20 \mathrm{~mL})$, and the organics were extracted with ethyl acetate $(3 \times 50 \mathrm{~mL})$. These were combined, washed with brine $(20 \mathrm{~mL})$, dried over $\mathrm{MgSO}_{4}$, filtered, and concentrated in vacuo to afford an orange oil. Further purification was achieved by FC (5-20\% ethyl acetate/petroleum ether) to afford the title compound, 41 , at $15 \%$ ethyl acetate/petroleum ether as a clear oil that foamed and crystallized to a white solid under high vacuum $(2.00 \mathrm{~g}, 3.93 \mathrm{mmol}, 79 \%)$. LC-MS (ESI+) Rt: $3.46 \mathrm{~min}$ $\left(254 \mathrm{~nm}\right.$, Method 1); $(\mathrm{m} / z)$ : $508.3\left[\mathrm{M}\left({ }^{35} \mathrm{Cl}\right)+\mathrm{H}\right]^{+} .{ }^{1} \mathrm{H} \quad \mathrm{NMR}$ $\left(400 \mathrm{MHz}, \mathrm{CDCl}_{3}\right) \delta: 8.02(\mathrm{~d}, J=8.5 \mathrm{~Hz}, 1 \mathrm{H}), 7.67(\mathrm{~s}, 1 \mathrm{H}), 7.31$ $(\mathrm{dd}, J=8.5,2.3 \mathrm{~Hz}), 7.16(\mathrm{~d}, J=2.2 \mathrm{~Hz}, 1 \mathrm{H}), 5.16(\mathrm{~s}, 1 \mathrm{H}), 3.12-3.04$ $(\mathrm{m}, 2 \mathrm{H}), 2.87-2.77(\mathrm{~m}, 1 \mathrm{H}), 2.72-2.66(\mathrm{~m}, 1 \mathrm{H}), 1.61(\mathrm{~s}, 9 \mathrm{H}), 1.43$ (s, 9H). ${ }^{13} \mathrm{C}$ NMR $\left(101 \mathrm{MHz}, \mathrm{CDCl}_{3}\right) \delta: 167.1,163.5,156.9,150.8$, $147.1,142.6,137.9,133.11,133.07,129.9,128.2,126.5,120.1,82.5$, $80.4,74.4,31.2,28.42,28.40,26.7$.

5-(2,7-Dichloro-4H-benzo[5,6]cyclohepta[1,2-d]thiazol-4-yl)pyrimidine-2,4(1H,3H)-dione (42). A solution of 41 (1.990 g, $3.91 \mathrm{mmol})$ in 1,4-dioxane $(19.5 \mathrm{~mL})$ was treated with acetic acid $(19.5 \mathrm{~mL})$ and heated to $140{ }^{\circ} \mathrm{C}$ with stirring in a microwave (MW) for $10 \mathrm{~min}$. This was concentrated in vacuo, and the residual acetic acid was azeotroped with toluene $(3 \times 50 \mathrm{~mL})$ to afford a brown solid. Purification was achieved by FC ( $5 \% \mathrm{MeOH} / \mathrm{DCM})$ to afford the title compound, 42, as an orange solid ( $851 \mathrm{mg}, 2.25 \mathrm{mmol}, 58 \%)$. LC-MS (ESI+) Rt: $2.71 \mathrm{~min}(254 \mathrm{~nm}$, Method 1); $(\mathrm{m} / z)$ : 378.1 $\left.\left[\mathrm{M}\left({ }^{35} \mathrm{Cl}\right)+\mathrm{H}\right]\right]^{+}$. ${ }^{1} \mathrm{H}$ NMR $(400 \mathrm{MHz}$, DMSO-d $) \delta: 11.08(\mathrm{~s}, 1 \mathrm{H})$, $10.60(\mathrm{~s}, 1 \mathrm{H}), 7.58(\mathrm{~d}, J=2.1 \mathrm{~Hz}, 1 \mathrm{H}), 7.53(\mathrm{~d}, J=8.4 \mathrm{~Hz}, 1 \mathrm{H}), 7.49$ $(\mathrm{dd}, J=8.3,2.2 \mathrm{~Hz}, 1 \mathrm{H}), 7.12(\mathrm{~d}, J=11.9 \mathrm{~Hz}, 1 \mathrm{H}) 7.07(\mathrm{~d}, J=$ $11.7 \mathrm{~Hz}, 1 \mathrm{H}), 6.63(\mathrm{~s}, 1 \mathrm{H}), 5.48(\mathrm{~s}, 1 \mathrm{H}) .{ }^{13} \mathrm{C}$ NMR $(101 \mathrm{MHz}$, DMSO- $\left.d_{6}\right) \delta: 163.4,151.2,150.6,149.0,138.6,136.2,134.9,133.1$, $131.8,131.5,130.1,129.8,120.5,108.3,43.7$.

5-(2,7-Dichloro-4H-benzo[5,6]cyclohepta[1,2-d]thiazol-4-yl)-1methylpyrimidine-2,4(1H,3H)-dione (43). A stirred suspension of $\mathbf{4 2}$ $(1.430 \mathrm{~g}, 3.80 \mathrm{mmol})$ in 1,2-dichloroethane $(40 \mathrm{~mL})$ under $\mathrm{N}_{2}$ was treated with $\mathrm{N}, \mathrm{O}$-bis (trimethylsilyl)trifluoroacetamide $(4.1 \mathrm{~mL}, 3.912 \mathrm{~g}$, $15.20 \mathrm{mmol}$ ) and heated to reflux for $18 \mathrm{~h}$, generating an orange solution. This was cooled, treated with iodomethane $(4.7 \mathrm{~mL}, 10.737 \mathrm{~g}, 76.0 \mathrm{mmol})$ and heated to $50{ }^{\circ} \mathrm{C}$ for $6 \mathrm{~h}$ until completion was observed by LC-MS. This was concentrated in vacuo to afford an orange oil, which was dissolved in $\mathrm{MeOH}$ and reconcentrated in vacuo to afford a yellow solid. This was triturated with diethyl ether $(3 \times 30 \mathrm{~mL})$ to afford the title compound, 43 an orange solid (1.466 g, $3.73 \mathrm{mmol}$, 98\%). LC-MS (ESI+) Rt: $2.83 \mathrm{~min}(254 \mathrm{~nm}$, Method 1); $(\mathrm{m} / z): 392.0$ $\left[\mathrm{M}\left({ }^{35} \mathrm{Cl}\right)+\mathrm{H}\right]^{+} .{ }^{1} \mathrm{H}$ NMR (400 MHz, DMSO- $\left.d_{6}\right) \delta: 11.27(\mathrm{~s}, 1 \mathrm{H})$, $7.56(\mathrm{~d}, J=2.3 \mathrm{~Hz}, 1 \mathrm{H}), 7.55(\mathrm{~d}, J=8.3 \mathrm{~Hz}, 1 \mathrm{H}), 7.48(\mathrm{dd}, J=8.3$, $2.2 \mathrm{~Hz}, 1 \mathrm{H}), 7.11(\mathrm{~d}, J=11.8 \mathrm{~Hz}, 1 \mathrm{H}), 7.05(\mathrm{~d}, J=11.7 \mathrm{~Hz}, 1 \mathrm{H}), 6.87$ $(\mathrm{d}, J=0.9 \mathrm{~Hz}, 1 \mathrm{H}), 5.50(\mathrm{~s}, 1 \mathrm{H}), 3.14(\mathrm{~s}, 3 \mathrm{H}) .{ }^{13} \mathrm{C} \mathrm{NMR}(101 \mathrm{MHz}$, DMSO- $\left.d_{6}\right) \delta$ : 163.1, 151.1, 150.6, 148.8, 143.2, 136.2, 134.8, 133.2, $132.1,131.8,131.5,130.2,129.7,120.6,108.4,43.9,36.0$.

5-(7-Chloro-2-phenyl-4H-benzo[5,6]cyclohepta[1,2-d]thiazol-4yl)-1-methylpyrimidine-2,4(1H,3H)-dione (44). A suspension of 43 ( $39 \mathrm{mg}, 0.099 \mathrm{mmol}$ ), phenylboronic acid (15 mg, $0.12 \mathrm{mmol}$ ), $\mathrm{Na}_{2} \mathrm{CO}_{3}$ (32 $\mathrm{mg}, 0.30 \mathrm{mmol}$ ), bis(triphenylphosphine) palladium(II)chloride $(7 \mathrm{mg}, 0.01 \mathrm{mmol})$ in 1,4 -dioxane $(0.8 \mathrm{~mL})$ and water $(0.2 \mathrm{~mL})$ was heated in a MW to $150{ }^{\circ} \mathrm{C}$ for 5 min, generating a black solution. This was concentrated directly onto silica and purified by FC (20-50\% ethyl acetate/toluene) to afford the title compound, 44, at 30\% ethyl acetate/toluene (29 mg, $0.067 \mathrm{mmol}, 68 \%)$. LC-MS (ESI+) Rt: $3.01 \mathrm{~min}\left(254 \mathrm{~nm}\right.$, Method 1); $(\mathrm{m} / z): 433.8\left[\mathrm{M}\left({ }^{35} \mathrm{Cl}\right)+\mathrm{H}\right]^{+}$. LC-MS (ESI+) Rt: $6.22 \min (254 \mathrm{~nm}$, Method 2); $(\mathrm{m} / z): 434.1$ $\left[\mathrm{M}\left({ }^{35} \mathrm{Cl}\right)+\mathrm{H}\right]^{+}($N.B. $85 \%$ purity at $254 \mathrm{~nm} ; 95 \%$ purity at $220 \mathrm{~nm})$. ${ }^{1} \mathrm{H}$ NMR $\left(400 \mathrm{MHz}\right.$, DMSO- $\left.d_{6}\right) \delta: 11.30(\mathrm{~s}, 1 \mathrm{H}), 7.96-7.94(\mathrm{~m}, 2 \mathrm{H})$, $7.61(\mathrm{~d}, J=8.4 \mathrm{~Hz}, 1 \mathrm{H}), 7.56(\mathrm{~d}, J=2.2 \mathrm{~Hz}, 1 \mathrm{H}), 7.53-7.49(\mathrm{~m}, 3 \mathrm{H})$, $7.48(\mathrm{dd}, J=8.3,2.3 \mathrm{~Hz}, 1 \mathrm{H}), 7.19(\mathrm{~d}, J=11.7 \mathrm{~Hz}, 1 \mathrm{H}), 7.11(\mathrm{~d}, J=$ $11.8 \mathrm{~Hz}, 1 \mathrm{H}), 7.06(\mathrm{~s}, 1 \mathrm{H}), 5.62(\mathrm{~s}, 1 \mathrm{H}), 3.18(\mathrm{~s}, 3 \mathrm{H}) .{ }^{13} \mathrm{C}$ NMR $\left(101 \mathrm{MHz}, \mathrm{DMSO}-d_{6}\right) \delta: 167.4,163.2,151.7,151.1,143.3,136.4$, 135.1, 133.2, 131.6, 131.1, 130.8, 130.0, 129.8, 129.3, 126.47, 126.44, 121.4, 110.0, 108.8, 43.9, 36.1.

5-(2-Amino-7-chloro-4H-benzo[5,6]cyclohepta[1,2-d]thiazol-4yl)-1-methylpyrimidine-2,4(1H,3H)-dione (45). A stirred solution of $43(140 \mathrm{mg}, 0.357 \mathrm{mmol})$ in 1,4-dioxane $(1 \mathrm{~mL})$ was treated with 880 $\mathrm{NH}_{3}(1 \mathrm{~mL})$ and heated in a MW to $100{ }^{\circ} \mathrm{C}$ with stirring for $4 \mathrm{~h}$. The RM was concentrated directly onto silica and purified by FC (5-20\% $\mathrm{MeOH}$ DCM with $0.1 \% 880 \mathrm{NH}_{3}$ ) to afford the title compound, 45, at $20 \% \mathrm{MeOH}$ DCM with $0.1 \% 880 \mathrm{NH}_{3}(68 \mathrm{mg}, 0.182 \mathrm{mmol}, 51 \%)$. LC-MS (ESI+) Rt: $2.37 \mathrm{~min}(254 \mathrm{~nm}$, Method 1); $(\mathrm{m} / \mathrm{z}): 372.8$ $\left[\mathrm{M}\left({ }^{35} \mathrm{Cl}\right)+\mathrm{H}\right]{ }^{+1} \mathrm{H}$ NMR $\left(400 \mathrm{MHz}\right.$, DMSO- $\left.d_{6}\right) \delta: 11.23(\mathrm{~s}, 1 \mathrm{H}), 7.44$ $(\mathrm{d}, J=8.7 \mathrm{~Hz}, 1 \mathrm{H}), 7.43(\mathrm{~d}, J=2.4 \mathrm{~Hz}, 1 \mathrm{H}), 7.37(\mathrm{dd}, J=8.2,2.3 \mathrm{~Hz})$, $7.36-7.32$ (br.s, $2 \mathrm{H}), 6.93(\mathrm{~s}, 1 \mathrm{H}), 6.79(\mathrm{~d}, J=11.6 \mathrm{~Hz}, 1 \mathrm{H}), 6.76$ $(\mathrm{d}, J=11.8 \mathrm{~Hz}, 1 \mathrm{H}), 5.22(\mathrm{~s}, 1 \mathrm{H}), 3.16(\mathrm{~s}, 3 \mathrm{H}) .{ }^{13} \mathrm{C}$ NMR $(101 \mathrm{MHz}$, DMSO-d $d_{6} \delta: 169.9,163.2,151.1,142.8,137.0,134.0,133.2,131.7$, 131.0, 129.4, 128.2, 126.0, 122.2, 117.3, 108.9, 43.9, 36.1

5-(7-Chloro-2-(phenylamino)-4H-benzo $[5,6]$ cyclohepta $[1,2-d]-$ thiazol-4-yl)-1-methylpyrimidine-2,4(1H,3H)-dione (46). A stirred solution of $43(75 \mathrm{mg}, 0.191 \mathrm{mmol})$ and aniline $(0.07 \mathrm{~mL}, 0.76 \mathrm{mmol})$ dissolved in 1,4-dioxane $(2 \mathrm{~mL}$ ) were treated with concd $\mathrm{HCl}$ (ca. two drops) and heated with stirring in a MW at $140{ }^{\circ} \mathrm{C}$ for $1 \mathrm{~h}$. This was evaporated directly onto silica and purified by FC $(2-5 \% \mathrm{MeOH} /$ DCM with $0.1 \% 880 \mathrm{NH}_{3}$ ) to afford the title compound, 46 , at $3 \%$ $\mathrm{MeOH} / \mathrm{DCM}$ with $0.1 \% 880 \mathrm{NH}_{3}$ as a pale-yellow solid $(63 \mathrm{mg}$, $0.14 \mathrm{mmol}, 74 \%)$. LC-MS (ESI+) Rt: $2.92 \mathrm{~min}(254 \mathrm{~nm}$, Method 1); $\left.(m / z): 448.9\left[\mathrm{M}\left({ }^{35} \mathrm{Cl}\right)+\mathrm{H}\right]\right]^{+} .{ }^{1} \mathrm{H}$ NMR $\left(400 \mathrm{MHz}, \mathrm{DMSO}-d_{6}\right) \delta$ : 11.46-11.03 (br.s, $1 \mathrm{H}), 10.73-10.19$ (br.s, $1 \mathrm{H}), 7.61(\mathrm{~d}, J=7.7 \mathrm{~Hz}$, $2 \mathrm{H}), 7.50(\mathrm{~d}, J=8.5 \mathrm{~Hz}, 1 \mathrm{H}), 7.47(\mathrm{~d}, J=2.1 \mathrm{~Hz}, 1 \mathrm{H}), 7.40(\mathrm{dd}, J=$ 8.3, 2.2 Hz, 1H), 7.33 (app.t, $J=7.9 \mathrm{~Hz}, 2 \mathrm{H}), 7.13(\mathrm{~s}, 1 \mathrm{H}), 6.98(\mathrm{t}, J=$ $7.4 \mathrm{~Hz}, 1 \mathrm{H}), 6.94-6.88(\mathrm{~m}, 2 \mathrm{H}), 5.29(\mathrm{~s}, 1 \mathrm{H}), 3.19(\mathrm{~s}, 3 \mathrm{H}) .{ }^{13} \mathrm{C}$ NMR $\left(101 \mathrm{MHz}, \mathrm{DMSO}-d_{6}\right) \delta: 164.3,151.3,147.6,143.52,141.1,137.0$, $134.4,132.7,131.1,129.5,129.4,128.6,127.75,127.73,122.2,121.9$, $118.5,117.8,108.8,43.6,36.2$.

5-(2-(Benzylamino)-7-chloro-4H-benzo[5,6]cyclohepta[1,2-d]thiazol-4-yl)-1-methylpyrimidine-2,4(1H,3H)-dione (47). Following general procedure $1,43(75 \mathrm{mg}, 0.191 \mathrm{mmol})$ was reacted with benzylamine $(0.08 \mathrm{~mL}, 0.76 \mathrm{mmol})$ to afford the title compound, 47 , at $4 \% \mathrm{MeOH} / \mathrm{DCM}$ with $0.1 \% 880 \mathrm{NH}_{3}$ as a pale-yellow solid $(45 \mathrm{mg}$, $0.097 \mathrm{mmol}, 51 \%)$. LC-MS (ESI+) Rt: $2.81 \mathrm{~min}(254 \mathrm{~nm}$, Method 1); $\left.(m / z): 462.9\left[\mathrm{M}\left({ }^{35} \mathrm{Cl}\right)+\mathrm{H}\right]\right]^{+} .{ }^{1} \mathrm{H}$ NMR $\left(400 \mathrm{MHz}, \mathrm{DMSO}-d_{6}\right) \delta$ : $11.25(\mathrm{~s}, 1 \mathrm{H}, \mathrm{s}, 1 \mathrm{H}), 8.41(\mathrm{t}, J=5.8 \mathrm{~Hz}, 1 \mathrm{H}), 7.45(\mathrm{~d}, J=8.5 \mathrm{~Hz}, 1 \mathrm{H})$, 
$7.43(\mathrm{~d}, J=2.3 \mathrm{~Hz}, 1 \mathrm{H}), 7.38-7.32(\mathrm{~m}, 5 \mathrm{H}), 7.29-7.24(\mathrm{~m}, 1 \mathrm{H}), 7.03$ $(\mathrm{s}, 1 \mathrm{H}), 6.82(\mathrm{~d}, J=11.6 \mathrm{~Hz}, 1 \mathrm{H}), 6.78(\mathrm{~d}, J=11.7 \mathrm{~Hz}, 1 \mathrm{H}), 5.22$ $(\mathrm{s}, 1 \mathrm{H}), 4.50(\mathrm{dd}, J=15.2,5.8 \mathrm{~Hz}, 1 \mathrm{H}), 4.42(\mathrm{dd}, J=15.3,5.8 \mathrm{~Hz}$, $1 \mathrm{H}), 3.16(\mathrm{~s}, 3 \mathrm{H}) .{ }^{13} \mathrm{C}$ NMR (101 MHz, DMSO-d $\left.{ }_{6}\right) \delta: 169.6,163.3$, $151.1,147.8,143.1,139.0,137.0,134.2,132.9,131.0,129.3,128.8$, $128.3,128.0,127.6,126.2,122.2,117.2,108.9,48.1,43.8,36.2$.

5-(7-Chloro-2-(phenethylamino)-4H-benzo[5,6]cyclohepta[1,2d] thiazol-4-yl)-1-methylpyrimidine-2,4(1H,3H)-dione (48). Following general procedure $1,43(75 \mathrm{mg}, 0.191 \mathrm{mmol})$ was reacted with phenethylamine $(0.10 \mathrm{~mL}, 0.76 \mathrm{mmol})$ to afford the title compound, 48, at $3 \% \mathrm{MeOH} / \mathrm{DCM}$ with $0.1 \% 880 \mathrm{NH}_{3}$ as a pale-yellow solid (51 mg, $0.10 \mathrm{mmol}, 54 \%)$. LC-MS (ESI+) Rt: $2.82 \mathrm{~min}(254 \mathrm{~nm}$, Method 1); $(m / z): 477.0\left[\mathrm{M}\left({ }^{35} \mathrm{Cl}\right)+\mathrm{H}\right]^{+}$. ${ }^{1} \mathrm{H}$ NMR (400 $\mathrm{MHz}$, DMSO- $\left.d_{6}\right) \delta: 11.23(\mathrm{~s}, 1 \mathrm{H}), 8.00(\mathrm{t}, J=5.4 \mathrm{~Hz}, 1 \mathrm{H}), 7.44(\mathrm{~d}, J=8.5 \mathrm{~Hz}$, $1 \mathrm{H}), 7.42(\mathrm{~d}, J=2.2 \mathrm{~Hz}, 1 \mathrm{H}), 7.36(\mathrm{dd}, J=8.3,2.3 \mathrm{~Hz}, 1 \mathrm{H}), 7.31-$ $7.23(\mathrm{~m}, 4 \mathrm{H}), 7.19(\mathrm{tt}, J=6.9,2.0 \mathrm{~Hz}, 1 \mathrm{H}), 6.98(\mathrm{~s}, 1 \mathrm{H}), 6.80(\mathrm{~d}, J=$ $11.6 \mathrm{~Hz}, 1 \mathrm{H}), 6.76(\mathrm{~d}, J=11.8 \mathrm{~Hz}, 1 \mathrm{H}), 5.22(\mathrm{~s}, 1 \mathrm{H}), 3.49-3.41(\mathrm{~m}$, $2 \mathrm{H}), 3.15(\mathrm{~s}, 3 \mathrm{H}), 2.86(\mathrm{t}, J=7.3 \mathrm{~Hz}, 2 \mathrm{H}) .{ }^{13} \mathrm{C}$ NMR $(101 \mathrm{MHz}$, DMSO- $\left._{6}\right) \delta: 169.5,163.3,151.1,148.0,143.0,139.7,137.1,134.1$, 133.1, 131.0, 129.32, 129.16, 128.8, 128.2, 126.6, 126.1, 122.2, 116.8, $108.9,46.2,43.9,36.2,35.1$.

5-(7-Chloro-2-(4-methylpiperazin-1-yl)-4H-benzo[5,6]cyclohepta[1,2-d]thiazol-4-yl)-1-methylpyrimidine-2,4(1H,3H)-dione (49). Following general procedure $1,43(75 \mathrm{mg}, 0.191 \mathrm{mmol})$ was reacted with $\mathrm{N}$-methylpiperazine $(0.08 \mathrm{~mL}, 0.76 \mathrm{mmol})$ to afford the title compound, 49, at $6 \% \mathrm{MeOH} / \mathrm{DCM}$ with $0.1 \% 880 \mathrm{NH}_{3}$ as a paleyellow solid (64 mg, $0.14 \mathrm{mmol}, 71 \%)$. LC-MS (ESI+) Rt: $2.13 \mathrm{~min}$ (254 nm, Method 1); $(m / z): 456.0\left[\mathrm{M}\left({ }^{35} \mathrm{Cl}\right)+\mathrm{H}\right]^{+}$. ${ }^{1} \mathrm{H}$ NMR $\left(400 \mathrm{MHz}, \mathrm{DMSO}-d_{6}\right) \delta: 11.25(\mathrm{~s}, 1 \mathrm{H}), 7.47(\mathrm{~d}, J=8.6 \mathrm{~Hz}, 1 \mathrm{H})$, $7.45(\mathrm{~d}, J=2.2 \mathrm{~Hz}, 1 \mathrm{H}), 7.38(\mathrm{dd}, J=8.3,2.3 \mathrm{~Hz}, 1 \mathrm{H}), 6.96(\mathrm{~s}, 1 \mathrm{H})$, $6.88(\mathrm{~d}, J=11.6 \mathrm{~Hz}, 1 \mathrm{H}), 6.82(\mathrm{~d}, J=11.7 \mathrm{~Hz}, 1 \mathrm{H}), 5.29(\mathrm{~s}, 1 \mathrm{H})$, $3.46-3.42(\mathrm{~m}, 4 \mathrm{H}), 3.16(\mathrm{~s}, 3 \mathrm{H}), 2.52-2.47(\mathrm{~m}, 4 \mathrm{H}), 2.29-2.25$ $(\mathrm{m}, 3 \mathrm{H}) .{ }^{13} \mathrm{C}$ NMR (101 MHz, DMSO-d $\left.d_{6}\right) \delta: 171.0,163.2,151.1$, $148.3,142.9,136.8,134.2,133.2,131.1,129.5,128.4,126.8,121.9$, $118.7,108.7,53.9,47.8,45.8,44.1,36.2$.

5-(7-Chloro-2-morpholino-4H-benzo[5,6]cyclohepta[1,2-d]thiazol-4-yl)-1-methylpyrimidine-2,4(1H,3H)-dione (50). Following general procedure $1,43(75 \mathrm{mg}, 0.191 \mathrm{mmol})$ was reacted with morpholine $(0.07 \mathrm{~mL}, 0.76 \mathrm{mmol})$ to afford the title compound, $\mathbf{5 0}$, at $3 \% \mathrm{MeOH} / \mathrm{DCM}$ with $0.1 \% 880 \mathrm{NH}_{3}$ as a pale-yellow solid $(65 \mathrm{mg}$, $0.147 \mathrm{mmol}, 77 \%)$. LC-MS (ESI+) Rt: $2.70 \mathrm{~min}(254 \mathrm{~nm}$, Method 1); $\left.(m / z): 443.1\left[\mathrm{M}\left({ }^{35} \mathrm{Cl}\right)+\mathrm{H}\right]\right]^{+} .{ }^{1} \mathrm{H}$ NMR $\left(400 \mathrm{MHz}, \mathrm{DMSO}-d_{6}\right)$ $\delta: 11.25(\mathrm{~s}, 1 \mathrm{H}), 7.47(\mathrm{~d}, J=8.5 \mathrm{~Hz}, 1 \mathrm{H}), 7.45(\mathrm{~d}, J=2.3 \mathrm{~Hz}$, $1 \mathrm{H}), 7.39(\mathrm{dd}, J=8.3,2.3 \mathrm{~Hz}, 1 \mathrm{H}), 6.97(\mathrm{~s}, 1 \mathrm{H}), 6.89(\mathrm{~d}, J=11.6 \mathrm{~Hz}$, $1 \mathrm{H}), 6.83(\mathrm{~d}, J=11.7 \mathrm{~Hz}, 1 \mathrm{H}), 5.29(\mathrm{~s}, 1 \mathrm{H}), 3.70(\mathrm{t}, J=4.9 \mathrm{~Hz}, 4 \mathrm{H})$, $3.40(\mathrm{t}, J=4.8 \mathrm{~Hz}, 4 \mathrm{H}), 3.16(\mathrm{~s}, 3 \mathrm{H}) .{ }^{13} \mathrm{C}$ NMR $\left(101 \mathrm{MHz}\right.$, DMSO-d $\left.d_{6}\right)$ $\delta: 171.4,163.3,151.1,148.2,142.9,136.8,134.2,133.2,131.1,129.5$, 128.4, 126.9, 121.9, 118.7, 108.7, 65.8, 49.1, 48.2, 36.2.

5-(7-Chloro-2-(piperidin-1-yl)-4H-benzo[5,6]cyclohepta[1,2-d]thiazol-4-yl)-1-methylpyrimidine-2,4(1H,3H)-dione (51). Following general procedure $1,43(75 \mathrm{mg}, 0.191 \mathrm{mmol})$ was reacted with piperidine $(0.08 \mathrm{~mL}, 0.76 \mathrm{mmol})$ to afford the title compound, $\mathbf{5 1}$, at $3 \% \mathrm{MeOH} / \mathrm{DCM}$ with $0.1 \% 880 \mathrm{NH}_{3}$ as a pale-yellow solid $(60 \mathrm{mg}$, $0.136 \mathrm{mmol}, 71 \%)$. LC-MS (ESI+) Rt: $2.88 \mathrm{~min}(254 \mathrm{~nm}$, Method 1); $\left.(m / z): 441.2\left[\mathrm{M}\left({ }^{35} \mathrm{Cl}\right)+\mathrm{H}\right]\right]^{+}$. ${ }^{1} \mathrm{H}$ NMR $\left(400 \mathrm{MHz}\right.$, DMSO- $\left.d_{6}\right)$ $\delta: 11.23(\mathrm{~s}, 1 \mathrm{H}), 7.46(\mathrm{~d}, J=8.5 \mathrm{~Hz}, 1 \mathrm{H}), 7.43(\mathrm{~d}, J=2.2 \mathrm{~Hz}$, $1 \mathrm{H}), 7.36(\mathrm{dd}, J=8.3,2.3 \mathrm{~Hz}, 1 \mathrm{H}), 6.94(\mathrm{~s}, 1 \mathrm{H}), 6.84(\mathrm{~d}, J=11.6 \mathrm{~Hz}$, $1 \mathrm{H}), 6.78(\mathrm{~d}, J=11.7 \mathrm{~Hz}, 1 \mathrm{H}), 5.26(\mathrm{~s}, 1 \mathrm{H}), 3.43-3.40(\mathrm{~m}, 4 \mathrm{H}), 3.14$ $(\mathrm{s}, 3 \mathrm{H}), 1.58(\mathrm{~s}, 6 \mathrm{H}) .{ }^{13} \mathrm{C}$ NMR (101 MHz, DMSO-d $)_{6} \delta: 171.1$, $163.3,151.1,148.5,142.8,136.9,134.1,133.3,131.1,129.4,128.3$, 126.3, 122.0, 118.0, 108.7, 49.2, 44.1, 36.2, 25.1, 23.9.

5-(7-Chloro-2-((2-methoxyethyl)amino)-4H-benzo[5,6]cyclohepta[1,2-d] thiazol-4-yl)-1-methylpyrimidine-2,4(1H,3H)dione (52). Following general procedure $1,43(75 \mathrm{mg}, 0.191 \mathrm{mmol})$ was reacted with 2-methoxyethylamine $(0.07 \mathrm{~mL}, 0.76 \mathrm{mmol})$ to afford the title compound, 52, at $4 \% \mathrm{MeOH} / \mathrm{DCM}$ with $0.1 \% 880$ $\mathrm{NH}_{3}$ as a pale-yellow solid (55 mg, $\left.0.128 \mathrm{mmol}, 67 \%\right)$. LC-MS (ESI+) Rt: $2.51 \mathrm{~min}\left(254 \mathrm{~nm}\right.$, Method 1); $(\mathrm{m} / z): 430.9\left[\mathrm{M}\left({ }^{35} \mathrm{Cl}\right)+\mathrm{H}\right]^{+}$. ${ }^{1} \mathrm{H}$ NMR $\left(400 \mathrm{MHz}, \mathrm{DMSO}-d_{6}\right) \delta: 11.23(\mathrm{~s}, 1 \mathrm{H}), 7.98(\mathrm{t}, J=5.3 \mathrm{~Hz}$, $1 \mathrm{H}), 7.44(\mathrm{~d}, J=8.6 \mathrm{~Hz}, 1 \mathrm{H}), 7.42(\mathrm{~d}, J=2.3 \mathrm{~Hz}, 1 \mathrm{H}), 7.36$ (dd, $J=8.3,2.3 \mathrm{~Hz}, 1 \mathrm{H}), 6.99(\mathrm{~s}, 1 \mathrm{H}), 6.79(\mathrm{~d}, J=11.6 \mathrm{~Hz}, 1 \mathrm{H}), 6.76$ $(\mathrm{d}, J=11.8 \mathrm{~Hz}, 1 \mathrm{H}), 5.21(\mathrm{~s}, 1 \mathrm{H}), 3.48-3.45(\mathrm{~m}, 2 \mathrm{H}), 3.26(\mathrm{~s}, 3 \mathrm{H})$, $3.24-3.23(\mathrm{~m}, 2 \mathrm{H}), 3.15(\mathrm{~s}, 3 \mathrm{H}) .{ }^{13} \mathrm{C}$ NMR $\left(101 \mathrm{MHz}, \mathrm{DMSO}-d_{6}\right) \delta$ : 169.6, 163.3, 161.6, 151.1, 147.8, 143.0, 137.0, 134.1, 131.0, 129.3, $128.2,126.1,122.3,116.9,108.8,70.6,58.4,44.2,43.9,36.1$.

5-(7-Chloro-2-((3-methoxypropyl)amino)-4H-benzo[5,6]cyclohepta[1,2-d] thiazol-4-yl)-1-methylpyrimidine-2,4(1H,3H)dione (53). Following general procedure $1,43(50 \mathrm{mg}, 0.127 \mathrm{mmol})$ was reacted with 3-methoxypropylamine $(0.05 \mathrm{~mL}, 0.51 \mathrm{mmol})$ to afford the title compound, 53, which was isolated at $5 \% \mathrm{MeOH} / \mathrm{DCM}$ as a pale-yellow solid ( $52 \mathrm{mg}, 0.117 \mathrm{mmol}, 92 \%)$. LC-MS (ESI+) Rt: $2.54 \mathrm{~min}\left(254 \mathrm{~nm}\right.$, Method 1); $(\mathrm{m} / z): 445.1\left[\mathrm{M}\left({ }^{35} \mathrm{Cl}\right)+\mathrm{H}\right]^{+}$. ${ }^{1} \mathrm{H}$ NMR $\left(400 \mathrm{MHz}, \mathrm{DMSO}-d_{6}\right) \delta: 11.23(\mathrm{~s}, 1 \mathrm{H}), 7.90(\mathrm{t}, J=5.5 \mathrm{~Hz}, 1 \mathrm{H}), 7.44$ $(\mathrm{d}, J=8.4 \mathrm{~Hz}, 1 \mathrm{H}), 7.42(\mathrm{~d}, J=2.2 \mathrm{~Hz}, 1 \mathrm{H}), 7.35(\mathrm{dd}, J=8.3,2.3 \mathrm{~Hz}$, $1 \mathrm{H}), 7.00(\mathrm{~s}, 1 \mathrm{H}), 6.80(\mathrm{~d}, J=11.6 \mathrm{~Hz}, 1 \mathrm{H}), 6.75(\mathrm{~d}, J=11.7 \mathrm{~Hz}, 1 \mathrm{H})$, $5.21(\mathrm{~s}, 1 \mathrm{H}), 3.37(\mathrm{t}, J=6.2 \mathrm{~Hz}, 2 \mathrm{H}), 3.28-3.23(\mathrm{~m}, 2 \mathrm{H}), 3.22$ (s, 3H), 3.15 (s, 3H), 1.76 (app.qi, $J=6.5 \mathrm{~Hz}, 2 \mathrm{H}) .{ }^{13} \mathrm{C}$ NMR $(101$ $\left.\mathrm{MHz}, \mathrm{DMSO}-d_{6}\right) \delta: 169.7,163.3,151.1,148.0,143.0,137.0,134.1$, 133.0, 131.0, 129.3, 128.2, 126.0, 122.3, 116.7, 108.9, 69.8, 58.4, 43.9, $41.9,36.1,29.2$.

5-(7-Chloro-2-((2-ethoxyethyl)amino)-4H-benzo[5,6]cyclohepta[1,2-d] thiazol-4-yl)-1-methylpyrimidine-2,4(1H,3H)-dione (54). Following general procedure $1,43(50 \mathrm{mg}, 0.127 \mathrm{mmol})$ was reacted with 2-ethoxyethylamine $(0.05 \mathrm{~mL}, 0.51 \mathrm{mmol})$ to afford the title compound, 54, at $5 \% \mathrm{MeOH} / \mathrm{DCM}$ as a pale-yellow solid $(51 \mathrm{mg}$, $0.115 \mathrm{mmol}$, 90\%). LC-MS (ESI+) Rt: $2.60 \mathrm{~min}(254 \mathrm{~nm}$, Method 1); $\left.(m / z): 445.1\left[\mathrm{M}\left({ }^{35} \mathrm{Cl}\right)+\mathrm{H}\right]\right]^{+}$. ${ }^{1} \mathrm{H}$ NMR $\left(400 \mathrm{MHz}, \mathrm{DMSO}-d_{6}\right) \delta$ : $11.24(\mathrm{~s}, 1 \mathrm{H}), 7.98$ (t, $J=5.5 \mathrm{~Hz}, 1 \mathrm{H}), 7.45(\mathrm{~d}, J=8.5 \mathrm{~Hz}, 1 \mathrm{H}), 7.43$ $(\mathrm{d}, J=2.3 \mathrm{~Hz}, 1 \mathrm{H}), 7.37(\mathrm{dd}, J=8.3,2.3 \mathrm{~Hz}, 1 \mathrm{H}), 7.01(\mathrm{~s}, 1 \mathrm{H}), 6.81$ $(\mathrm{d}, J=11.6 \mathrm{~Hz}, 1 \mathrm{H}), 6.77(\mathrm{~d}, J=11.7 \mathrm{~Hz}, 1 \mathrm{H}), 5.23(\mathrm{~s}, 1 \mathrm{H}), 3.51(\mathrm{t}, J=$ $5.7 \mathrm{~Hz}, 2 \mathrm{H}), 3.45(\mathrm{q}, J=7.0 \mathrm{~Hz}, 2 \mathrm{H}), 3.43-3.37(\mathrm{~m}, 2 \mathrm{H}), 3.17(\mathrm{~s}, 3 \mathrm{H})$, $1.11(\mathrm{t}, J=7.0 \mathrm{~Hz}, 3 \mathrm{H}) .{ }^{13} \mathrm{C}$ NMR $\left(101 \mathrm{MHz}\right.$, DMSO- $\left.d_{6}\right) \delta: 169.6$, $163.3,151.1,147.8,143.0,137.0,134.1,133.1,131.0,129.3,128.2$, 126.1, 122.3, 116.9, 108.9, 68.5, 65.9, 44.5, 43.9, 36.1, 15.6.

5-(7-Chloro-2-((2-isopropoxyethyl)amino)-4H-benzo[5,6]cyclohepta[1,2-d] thiazol-4-yl)-1-methylpyrimidine-2,4(1H,3H)dione (55). Following general procedure $1,43(50 \mathrm{mg}, 0.127 \mathrm{mmol})$ was reacted with 2-isopropoxyethylamine $(0.06 \mathrm{~mL}, 0.51 \mathrm{mmol})$ to afford the title compound, 55, which was isolated at 5\% MeOH/DCM as a pale-yellow solid ( $49 \mathrm{mg}, 0.107 \mathrm{mmol}, 84 \%)$. LC-MS (ESI+) Rt: $2.68 \mathrm{~min}\left(254 \mathrm{~nm}\right.$, Method 1); $(\mathrm{m} / z): 459.2\left[\mathrm{M}\left({ }^{35} \mathrm{Cl}\right)+\mathrm{H}\right]^{+}$. ${ }^{1} \mathrm{H}$ NMR $\left(400 \mathrm{MHz}, \mathrm{DMSO}-d_{6}\right) \delta: 11.24(\mathrm{~s}, 1 \mathrm{H}), 7.94(\mathrm{t}, J=5.5 \mathrm{~Hz}, 1 \mathrm{H}), 7.45$ (d, $J=8.4 \mathrm{~Hz}, 1 \mathrm{H}), 7.43(\mathrm{~d}, J=2.1 \mathrm{~Hz}, 1 \mathrm{H}), 7.37(\mathrm{dd}, J=8.3,2.2 \mathrm{~Hz}$, $1 \mathrm{H}), 7.00(\mathrm{~s}, 1 \mathrm{H}), 6.81(\mathrm{~d}, J=11.6 \mathrm{~Hz}, 1 \mathrm{H}), 6.77(\mathrm{~d}, J=11.7 \mathrm{~Hz}, 1 \mathrm{H})$, $5.23(\mathrm{~s}, 1 \mathrm{H}), 3.57$ (sep., $J=6.1 \mathrm{~Hz}, 1 \mathrm{H}), 3.52-3.49(\mathrm{~m}, 2 \mathrm{H}), 3.42-$ $3.36(\mathrm{~m}, 2 \mathrm{H}), 3.16(\mathrm{~s}, 3 \mathrm{H}), 1.09(\mathrm{~d}, J=6.1 \mathrm{~Hz}, 6 \mathrm{H}) .{ }^{13} \mathrm{C}$ NMR $(101$ MHz, DMSO- $\left.d_{6}\right) \delta: 169.6,163.3,151.1,147.8,143.0,137.0,134.1$, $133.1,131.0,129.3,128.2,126.0,122.3,116.9,108.9,71.3,66.1,44.9$ 43.9, 36.1, 22.51, 22.49.

5-(7-Chloro-2-((2-phenoxyethyl)amino)-4H-benzo[5,6]cyclohepta[1,2-d] thiazol-4-yl)-1-methylpyrimidine-2,4(1H,3H)dione (56). Following general procedure $1,43(50 \mathrm{mg}, 0.127 \mathrm{mmol})$ was reacted with 2-phenoxyethylamine $(0.07 \mathrm{~mL}, 0.51 \mathrm{mmol})$ to afford the title compound, 56, at $4 \% \mathrm{MeOH} / \mathrm{DCM}$ as a pale-yellow solid (53 mg, $0.108 \mathrm{mmol}, 85 \%)$. LC-MS (ESI+) Rt: $2.85 \mathrm{~min}(254 \mathrm{~nm}$, Method 1); $(\mathrm{m} / z)$ : $\left.483.0\left[\mathrm{M}\left({ }^{35} \mathrm{Cl}\right)+\mathrm{H}\right]\right]^{+}$. ${ }^{1} \mathrm{H}$ NMR $(400 \mathrm{MHz}$, DMSO- $\left.d_{6}\right) \delta: 11.24(\mathrm{~s}, 1 \mathrm{H}), 8.17(\mathrm{t}, J=5.5 \mathrm{~Hz}, 1 \mathrm{H}), 7.46(\mathrm{~d}, J=$ $8.4 \mathrm{~Hz}, 1 \mathrm{H}), 7.44(\mathrm{~d}, J=2.2 \mathrm{~Hz}, 1 \mathrm{H}), 7.38(\mathrm{dd}, J=8.3,2.2 \mathrm{~Hz}, 1 \mathrm{H})$, $7.29(\mathrm{dd}, J=8.5,7.5 \mathrm{~Hz}, 2 \mathrm{H}), 7.00(\mathrm{~s}, 1 \mathrm{H}), 7.01-6.92(\mathrm{~m}, 3 \mathrm{H}), 6.82$ $(\mathrm{d}, J=11.6 \mathrm{~Hz}, 1 \mathrm{H}), 6.79(\mathrm{~d}, J=11.7 \mathrm{~Hz}, 1 \mathrm{H}), 5.26(\mathrm{~s}, 1 \mathrm{H}), 4.13(\mathrm{t}, J=$ $5.4 \mathrm{~Hz}, 2 \mathrm{H}), 3.64$ (app.q, $J=5.1 \mathrm{~Hz}, 2 \mathrm{H}), 3.16(\mathrm{~s}, 3 \mathrm{H}) .{ }^{13} \mathrm{C}$ NMR $(101$ MHz, DMSO- $\left.d_{6}\right) \delta: 169.4,163.3,158.8,151.1,148.3,147.7,143.0$, $137.0,134.1,131.0,130.0,129.4,128.3,126.2,122.2,117.2,114.9$, $108.8,66.2,43.9,36.1$.

Ethyl 2-((5-(2,7-Dichloro-4H-benzo[5,6]cyclohepta[1,2-d]thiazol4-yl)-2,4-dioxo-3,4-dihydropyrimidin-1(2H)-yl)methyl)thiazole-4carboxylate (57). A stirred suspension of $42(851 \mathrm{mg}, 2.25 \mathrm{mmol})$ in 1,2-dichloroethane $(20 \mathrm{~mL})$ was treated with $\mathrm{N}, \mathrm{O}$-bis(trimethylsilyl)trifluoroacetamide $(1.8 \mathrm{~mL}, 6.75 \mathrm{mmol})$ and heated to reflux for $18 \mathrm{~h}$ under $\mathrm{N}_{2}$, generating an orange solution. This was then treated with ethyl 2-(bromomethyl)thiazole-4-carboxylate $(591 \mathrm{mg}, 2.36 \mathrm{mmol}$ ) 
that had been dissolved in 1,2-dichloroethane $(3 \mathrm{~mL})$ and refluxed for $72 \mathrm{~h}$. This was concentrated in vacuo to a gum, treated with $\mathrm{MeOH}$ $(20 \mathrm{~mL})$, and then reconcentrated in vacuo to a brown solid. This was triturated with diethyl ether $(3 \times 50 \mathrm{~mL})$ to give the title compound, 57, as a brown solid (1.125 g, $2.06 \mathrm{mmol}$, 91\%). LC-MS (ESI+) Rt: $2.95 \mathrm{~min}(254 \mathrm{~nm}$, Method 1$) ;(\mathrm{m} / z): 546.9\left[\mathrm{M}\left({ }^{35} \mathrm{Cl}\right)+\mathrm{H}\right]^{+}$. ${ }^{1} \mathrm{H}$ NMR $\left(400 \mathrm{MHz}, \mathrm{DMSO}-d_{6}\right) \delta: 11.49(\mathrm{~s}, 1 \mathrm{H}), 8.50(\mathrm{~s}, 1 \mathrm{H}), 7.59(\mathrm{~d}, J=$ $8.5 \mathrm{~Hz}, 1 \mathrm{H}), 7.54(\mathrm{~d}, J=2.2 \mathrm{~Hz}, 1 \mathrm{H}), 7.51(\mathrm{dd}, J=8.2,2.3 \mathrm{~Hz}, 1 \mathrm{H})$, $7.03(\mathrm{~d}, J=11.9 \mathrm{~Hz}, 1 \mathrm{H}), 7.03(\mathrm{~d}, J=1.1 \mathrm{~Hz}, 1 \mathrm{H}), 6.96(\mathrm{~d}, J=11.7 \mathrm{~Hz}$, $1 \mathrm{H}), 5.55(\mathrm{~d}, J=0.9 \mathrm{~Hz}, 1 \mathrm{H}), 5.20(\mathrm{~d}, J=15.8 \mathrm{~Hz}, 1 \mathrm{H}), 5.16(\mathrm{~d}, J=$ $15.9 \mathrm{~Hz}, 1 \mathrm{H}), 4.34(\mathrm{q}, J=7.1 \mathrm{~Hz}, 2 \mathrm{H}), 1.33(\mathrm{t}, J=7.1 \mathrm{~Hz}, 3 \mathrm{H})$. ${ }^{13} \mathrm{C}$ NMR (101 MHz, DMSO- $\left.d_{6}\right) \delta: 166.6,162.7,161.0,150.67$, $150.55,148.6,146.4,142.0,136.2,134.5,133.3,132.00,131.81,131.5$, $130.5,130.1,129.7,120.6,109.0,61.4,48.4,44.1,14.7$.

Ethyl 2-((5-(7-Chloro-2-((2-methoxyethyl)amino)-4H-benzo[5,6]cyclohepta[1,2-d]thiazol-4-yl)-2,4-dioxo-3,4-dihydropyrimidin$1(2 \mathrm{H})$-yl)methyl)thiazole-4-carboxylate (58). Following general procedure $1,57(400 \mathrm{mg}, 0.73 \mathrm{mmol})$ was reacted with 2-methoxyethylamine $(0.25 \mathrm{~mL}, 2.92 \mathrm{mmol})$ to afford the title compound, 58, at $3 \%$ $\mathrm{MeOH} / \mathrm{DCM}$ as a pale-yellow solid (250 $\mathrm{mg}, 0.43 \mathrm{mmol}, 59 \%)$. LC-MS (ESI+) Rt: $2.69 \mathrm{~min}(254 \mathrm{~nm}$, Method 1); $(\mathrm{m} / z): 585.8$ $\left.\left[\mathrm{M}\left({ }^{35} \mathrm{Cl}\right)+\mathrm{H}\right]\right]^{+} .{ }^{1} \mathrm{H}$ NMR $\left(500 \mathrm{MHz}\right.$, DMSO- $\left.d_{6}\right) \delta: 11.46(\mathrm{~s}, 1 \mathrm{H})$, $8.51(\mathrm{~s}, 1 \mathrm{H}), 7.97(\mathrm{t}, J=5.4 \mathrm{~Hz}, 1 \mathrm{H}), 7.46(\mathrm{~d}, J=8.4 \mathrm{~Hz}, 1 \mathrm{H}), 7.39$ $(\mathrm{d}, J=2.1 \mathrm{~Hz}, 1 \mathrm{H}), 7.37(\mathrm{dd}, J=8.1,2.3 \mathrm{~Hz}, 1 \mathrm{H}), 7.18(\mathrm{~s}, 1 \mathrm{H}), 6.68$ $(\mathrm{d}, J=11.6 \mathrm{~Hz}, 1 \mathrm{H}), 6.65(\mathrm{~d}, J=11.7 \mathrm{~Hz}, 1 \mathrm{H}), 5.27(\mathrm{~d}, J=15.9 \mathrm{~Hz}$, $1 \mathrm{H}), 5.22(\mathrm{~s}, 1 \mathrm{H}), 5.15(\mathrm{~d}, J=15.9 \mathrm{~Hz}, 1 \mathrm{H}), 4.34(\mathrm{q}, J=7.0 \mathrm{~Hz}, 2 \mathrm{H})$, $3.46-3.44(\mathrm{~m}, 2 \mathrm{H}), 3.39$ (app.q, $J=5.6 \mathrm{~Hz}, 2 \mathrm{H}), 3.25(\mathrm{~s}, 3 \mathrm{H}), 1.33$ $(\mathrm{t}, J=7.1 \mathrm{~Hz}, 3 \mathrm{H}) .{ }^{13} \mathrm{C}$ NMR $\left(126 \mathrm{MHz}, \mathrm{DMSO}-d_{6}\right) \delta: 169.5,166.5$, $163.0,161.0,150.7,147.4,146.3,141.8,137.0,133.8,133.0,131.0$, $130.6,129.2,128.2,126.0,122.3,116.9,109.3,70.6,61.3,58.4,48.5$, $44.16,43.99,14.7$

Ethyl 2-((5-(7-Chloro-2-((2-methoxyethyl)amino)-4H-benzo[5,6]cyclohepta[1,2-d]thiazol-4-yl)-2-oxo-4-thioxo-3,4-dihydropyrimidin-1(2H)-yl)methyl)thiazole-4-carboxylate (59). Following general procedure $2,58(240 \mathrm{mg}, 0.41 \mathrm{mmol})$ was converted to the title compound, 59, which was isolated at $2 \% \mathrm{MeOH} / \mathrm{DCM}$ as a yellow solid (200 mg, $0.33 \mathrm{mmol}, 81 \%)$. LC-MS (ESI+) Rt: $2.87 \mathrm{~min}(254 \mathrm{~nm}$, Method 1); $(m / z): 601.9\left[\mathrm{M}\left({ }^{35} \mathrm{Cl}\right)+\mathrm{H}\right]^{+} .{ }^{1} \mathrm{H}$ NMR $(500 \mathrm{MHz}$, DMSO- $\left.d_{6}\right) \delta: 12.87(\mathrm{~s}, 1 \mathrm{H}), 8.55(\mathrm{~s}, 1 \mathrm{H}), 8.02-7.98$ (br.s, $\left.1 \mathrm{H}\right), 7.74$ $(\mathrm{s}, 1 \mathrm{H}), 7.56(\mathrm{~d}, J=8.5 \mathrm{~Hz}, 1 \mathrm{H}), 7.40(\mathrm{~d}, J=2.3 \mathrm{~Hz}, 1 \mathrm{H}), 7.36(\mathrm{dd}, J=$ 8.4, $2.3 \mathrm{~Hz}, 1 \mathrm{H}), 6.75(\mathrm{~s}, 2 \mathrm{H}), 5.65(\mathrm{~s}, 1 \mathrm{H}), 5.39(\mathrm{~d}, J=15.8 \mathrm{~Hz}, 1 \mathrm{H})$, $5.26(\mathrm{~d}, J=15.8 \mathrm{~Hz}, 1 \mathrm{H}), 4.35(\mathrm{q}, J=7.0 \mathrm{~Hz}, 2 \mathrm{H}), 3.42-3.40(\mathrm{~m}$, $2 \mathrm{H}), 3.37-3.35(\mathrm{~m}, 2 \mathrm{H}), 3.23(\mathrm{~s}, 3 \mathrm{H}), 1.34(\mathrm{t}, J=7.1 \mathrm{~Hz}, 3 \mathrm{H})$. ${ }^{13} \mathrm{C}$ NMR $\left(126 \mathrm{MHz}\right.$, DMSO- $\left.d_{6}\right) \delta: 189.8,169.3,165.5,161.0,147.9$, $146.4,141.9,137.5,133.7,132.1,130.93,130.81,128.9,127.8,126.7$, 122.7, 117.6, 116.7, 70.6, 61.4, 58.4, 49.2, 45.3, 44.1, 14.7.

3-((5-(2,8-Dimethyl-5H-dibenzo[a,d][7]annulen-5-yl)-2-oxo-4thioxo-3,4-dihydropyrimidin-1 (2H)-yl) methyl)-5(methoxycarbonyl)benzoic Acid (61) and 5-((5-(2,8-Dimethyl-5Hdibenzo[a,d][7]annulen-5-yl)-2-oxo-4-thioxo-3,4-dihydropyrimidin-1(2H)-yl)methyl)isophthalic Acid (60). Step A: N,O-Bis(trimethylsilyl)trifluoroacetamide $(0.76 \mathrm{~g}, 2.96 \mathrm{mmol})$ was added to a stirred suspension of 5-(2,8-dimethyl-5H-dibenzo[a,d][7]annulen-5yl)pyrimidine-2,4 $(1 \mathrm{H}, 3 \mathrm{H})$-dione $^{31}(0.49 \mathrm{~g}, 1.48 \mathrm{mmol})$ in 1,2-dichloroethane $(6 \mathrm{~mL})$, and the reaction mixture was heated under reflux under nitrogen. After $1.5 \mathrm{~h}$, dimethyl 5-(bromomethyl)isophthalate $(0.43 \mathrm{~g}, 1.48 \mathrm{mmol})$ was added. The reaction mixture was heated under reflux for a further $16 \mathrm{~h}$. After the reaction mixture cooled, the volatiles were removed under vacuum. Purification was by silica-gel chromatography, and the eluent was ethyl acetate/petroleum ether, 50:50, which gave dimethyl 5-((5-(2,8-dimethyl-5H-dibenzo[a,d][7]annulen-5-yl)-2,4-dioxo-3,4-dihydropyrimidin-1 $(2 \mathrm{H})$-yl)methyl)isophthalate $(0.7 \mathrm{~g}, 1.3 \mathrm{mmol}, 87 \%)$. LC-MS (ESI+) Rt: $3.10(254 \mathrm{~nm}$, Method 1); $(\mathrm{m} / \mathrm{z})$ : $537.2(\mathrm{M}+1)$. Step B: Lawesson's reagent $(0.8 \mathrm{~g}$, $2 \mathrm{mmol})$ and the product from step A $(0.7 \mathrm{~g}, 1.3 \mathrm{mmol})$ in 1,4-dioxane $(8 \mathrm{mmol})$ were heated under reflux under nitrogen. After $16 \mathrm{~h}$, the volatiles were evaporated under vacuum. Purification was by silica-gel chromatography, and the eluent was ethyl acetate/toluene, 30:70, which gave dimethyl 5-( 5 -(2,8-dimethyl-5H-dibenzo[ $a, d][7]$ annulen5-yl)-2-oxo-4-thioxo-3,4-dihydropyrimidin-1(2H)-yl)methyl)isophthalate $(0.4 \mathrm{~g}, 0.72 \mathrm{mmol}, 55 \%)$ as a yellow solid. LC-MS (ESI+)
Rt: 3.19 (254 nm, Method 1); $(m / z): 553.7(\mathrm{M}+1)$. Step C: A sodium hydroxide solution $(0.4 \mathrm{mmol}, 0.4 \mathrm{~mL}$ of a $1 \mathrm{M}$ solution in water) was added to a mixture of the product from step $B(0.11 \mathrm{~g}, 0.2 \mathrm{mmol})$ in methanol $(1 \mathrm{~mL})$, toluene $(0.5 \mathrm{~mL})$, and water $(0.5 \mathrm{~mL})$. After $4 \mathrm{~h}$, the reaction mixture was partitioned between $2 \mathrm{M}$ hydrochloric acid and ethyl acetate. The ethyl acetate layer was washed with brine, dried (magnesium sulfate), filtered, and evaporated in vacuo. Purification was by silica-gel chromatography, and the eluents were $2 \%$ acetic acid in ethyl acetate/toluene (25:75 and then 80:20). Yield of 61: $0.07 \mathrm{~g}$, $0.13 \mathrm{mmol}, 65 \%$ (yellow solid). ${ }^{1} \mathrm{H}$ NMR (400 MHz, DMSO) $\delta: 13.47$ $(\mathrm{s}, 1 \mathrm{H}), 12.62(\mathrm{~s}, 1 \mathrm{H}), 8.49(\mathrm{tt}, \mathrm{J}=3.9,1.7 \mathrm{~Hz}, 1 \mathrm{H}), 8.12(\mathrm{td}, \mathrm{J}=13.7$, $1.6 \mathrm{~Hz}, 2 \mathrm{H}), 7.45(\mathrm{~d}, \mathrm{~J}=7.8 \mathrm{~Hz}, 2 \mathrm{H}), 7.15(\mathrm{dd}, \mathrm{J}=7.9,1.9 \mathrm{~Hz}, 2 \mathrm{H})$, $7.09(\mathrm{td}, \mathrm{J}=5.1,1.9 \mathrm{~Hz}, 2 \mathrm{H}), 7.02(\mathrm{~s}, 1 \mathrm{H}), 6.70(\mathrm{~s}, 2 \mathrm{H}), 5.72(\mathrm{~s}, 1 \mathrm{H})$, $4.99(\mathrm{t}, \mathrm{J}=4.0 \mathrm{~Hz}, 2 \mathrm{H}), 3.97$ (s, 3H), 2.27 (s, 6H). LC-MS (ESI+) Rt: $3.05(254 \mathrm{~nm}) ;(\mathrm{m} / z): 539.6(\mathrm{M}+1)$. Yield of $60: 0.02 \mathrm{~g}, 0.038 \mathrm{mmol}$, $19 \%$ (yellow solid). ${ }^{1} \mathrm{H}$ NMR (400 MHz, DMSO) $\delta: 13.47$ (s, 2H), $12.62(\mathrm{~s}, 1 \mathrm{H}), 8.49(\mathrm{q}, \mathrm{J}=2.0 \mathrm{~Hz}, 1 \mathrm{H}), 8.09(\mathrm{~d}, \mathrm{~J}=1.6 \mathrm{~Hz}, 2 \mathrm{H}), 7.45$ $(\mathrm{d}, \mathrm{J}=7.9 \mathrm{~Hz}, 2 \mathrm{H}), 7.15(\mathrm{dd}, \mathrm{J}=7.9,1.9 \mathrm{~Hz}, 2 \mathrm{H}), 7.07(\mathrm{~d}, \mathrm{~J}=1.9 \mathrm{~Hz}$, $2 \mathrm{H}), 7.02(\mathrm{~s}, 1 \mathrm{H}), 6.70(\mathrm{~s}, 2 \mathrm{H}), 5.72(\mathrm{~s}, 1 \mathrm{H}), 4.98(\mathrm{~s}, 2 \mathrm{H}), 2.27(\mathrm{~s}, 6 \mathrm{H})$. LC-MS (ESI+) Rt: $2.81(254 \mathrm{~nm}$, Method 1); $(\mathrm{m} / \mathrm{z}): 525.2(\mathrm{M}+1)$.

Methyl 3-((2-((tert-Butoxycarbonyl)amino)ethyl)carbamoyl)-5((5-(2,8-dimethyl-5H-dibenzo[a,d][7]annulen-5-yl)-2-oxo-4-thioxo3,4-dihydropyrimidin-1(2H)-yl)methyl)benzoate (62). PyBroP $(0.19 \mathrm{~g}, 0.4 \mathrm{mmol})$ was added to a stirred solution of $3-((5-(2,8-$ dimethyl-5H-dibenzo[ $a, d][7]$ annulen-5-yl)-2-oxo-4-thioxo-3,4-dihydropyrimidin-1(2H)-yl)methyl)-5-(methoxycarbonyl)benzoic acid $(0.15 \mathrm{~g}, 0.28 \mathrm{mmol})$, tert-butyl (2-aminoethyl)carbamate (0.058 g, $0.36 \mathrm{mmol})$, and triethylamine $(0.15 \mathrm{~g}, 1.5 \mathrm{mmol})$ in DMF $(2 \mathrm{~mL})$. After $0.5 \mathrm{~h}$, the solution was diluted with ethyl acetate $(15 \mathrm{~mL})$ and washed with water $(2 \times 4 \mathrm{~mL})$ and brine $(1 \times 4 \mathrm{~mL})$. The solution was evaporated to dryness under reduced pressure. Purification was by silica-gel chromatography, and the eluent was 70:30 ethyl acetate/ petroleum ether (40-60). Yield: $0.1 \mathrm{~g}, 0.15 \mathrm{mmol}, 53 \%$ (yellow solid). ${ }^{1} \mathrm{H} \mathrm{NMR}\left(400 \mathrm{MHz}, \mathrm{CDCl}_{3}\right) \delta: 9.47(\mathrm{~s}, 1 \mathrm{H}), 8.50(\mathrm{~s}, 1 \mathrm{H}), 8.04(\mathrm{dt}, \mathrm{J}=$ 7.1, $1.8 \mathrm{~Hz}, 2 \mathrm{H}), 7.63-7.58(\mathrm{~m}, 1 \mathrm{H}), 7.50(\mathrm{~d}, \mathrm{~J}=7.8 \mathrm{~Hz}, 2 \mathrm{H}), 7.16$ $(\mathrm{dd}, \mathrm{J}=7.9,1.5 \mathrm{~Hz}, 1 \mathrm{H}), 7.07(\mathrm{~s}, 1 \mathrm{H}), 6.90(\mathrm{~s}, 1 \mathrm{H}), 6.68(\mathrm{~s}, 2 \mathrm{H}), 5.74$ (s, 1H), $5.33(\mathrm{~s}, 1 \mathrm{H}), 5.10-5.05(\mathrm{~m}, 1 \mathrm{H}), 4.79(\mathrm{~s}, 2 \mathrm{H}), 4.02(\mathrm{~s}, 3 \mathrm{H})$, $3.68-3.60(\mathrm{~m}, 2 \mathrm{H}), 3.50-3.44(\mathrm{~m}, 2 \mathrm{H}), 2.32(\mathrm{~s}, 6 \mathrm{H}), 1.44(\mathrm{~s}, 8 \mathrm{H})$. LC-MS (ESI+) Rt: 3.14 (254 nm, Method 1); $(\mathrm{m} / z): 681.2(\mathrm{M}+1)$

Methyl 3-((2,2-Dimethyl-4-oxo-3,8,11-trioxa-5-azatridecan-13yl)carbamoyl)-5-((5-(2,8-dimethyl-5H-dibenzo[a,d][7]annulen-5-yl)2-oxo-4-thioxo-3,4-dihydropyrimidin-1(2H)-yl)methyl)benzoate (63). PyBroP $(0.11 \mathrm{~g}, 0.24 \mathrm{mmol})$ was added to a stirred solution of 3-((5-(2,8-dimethyl-5H-dibenzo[a,d] [7] annulen-5-yl)-2-oxo-4-thioxo3,4-dihydropyrimidin-1(2H)-yl)methyl)-5-(methoxycarbonyl)benzoic acid (0.1 g, $0.186 \mathrm{mmol})$, tert-butyl (2-(2-(2-aminoethoxy)ethoxy)ethyl)carbamate $(0.05 \mathrm{~g}, 0.2 \mathrm{mmol})$, and triethylamine $(0.1 \mathrm{~g}, 1 \mathrm{mmol})$ in DMF $(2 \mathrm{~mL})$. After $0.5 \mathrm{~h}$, the solution was diluted with ethyl acetate $(15 \mathrm{~mL})$ and washed with water $(2 \times 4 \mathrm{~mL})$ and brine $(1 \times 4 \mathrm{~mL})$. The solution was evaporate to dryness under reduced pressure. Purification was by silica-gel chromatography, and the eluent was ethyl acetate. Yield: $0.09 \mathrm{~g}, 0.12 \mathrm{mmol}, 63 \%$ (yellow solid). ${ }^{1} \mathrm{H}$ NMR (400 MHz, DMSO) $\delta: 12.61(\mathrm{~s}, 1 \mathrm{H}), 8.89(\mathrm{t}, \mathrm{J}=5.5 \mathrm{~Hz}, 1 \mathrm{H}), 8.48$ $(\mathrm{t}, \mathrm{J}=1.6 \mathrm{~Hz}, 1 \mathrm{H}), 8.05(\mathrm{t}, \mathrm{J}=1.7 \mathrm{~Hz}, 1 \mathrm{H}), 8.00(\mathrm{t}, \mathrm{J}=1.6 \mathrm{~Hz}, 1 \mathrm{H})$, $7.44(\mathrm{~d}, \mathrm{~J}=7.9 \mathrm{~Hz}, 2 \mathrm{H}), 7.14(\mathrm{ddd}, \mathrm{J}=7.8,1.9,0.8 \mathrm{~Hz}, 2 \mathrm{H}), 7.05$ $(\mathrm{dd}, \mathrm{J}=1.9,0.8 \mathrm{~Hz}, 2 \mathrm{H}), 6.94(\mathrm{~s}, 1 \mathrm{H}), 6.76(\mathrm{t}, \mathrm{J}=5.6 \mathrm{~Hz}, 1 \mathrm{H}), 6.62$ $(\mathrm{s}, 2 \mathrm{H}), 5.71(\mathrm{~s}, 1 \mathrm{H}), 4.95(\mathrm{~s}, 2 \mathrm{H}), 3.97(\mathrm{~s}, 3 \mathrm{H}), 3.65-3.56(\mathrm{~m}, 4 \mathrm{H})$, $3.56-3.47(\mathrm{~m}, 4 \mathrm{H}), 3.39(\mathrm{t}, \mathrm{J}=12.3 \mathrm{~Hz}, 1 \mathrm{H}), 3.33-3.30(\mathrm{~m}, 1 \mathrm{H}), 3.07$ $(\mathrm{q}, \mathrm{J}=6.0 \mathrm{~Hz}, 2 \mathrm{H}), 2.27$ (s, 6H), 1.37 (s, 9H). LC-MS (ESI+) Rt: 3.19 (254 nm, Method 1); $(\mathrm{m} / \mathrm{z}): 769.6(\mathrm{M}+1)$

tert-Butyl (2-(3-)(2H-Tetrazol-5-yl)carbamoyl)-5-((5-(2,8-dimethyl-5H-dibenzo[a,d][7]annulen-5-yl)-2-oxo-4-thioxo-3,4-dihydropyrimidin-1(2H)-yl)methyl)benzamido)ethyl)carbamate (64). Lithium hydroxide $(0.04 \mathrm{~g}, 1.6 \mathrm{mmol})$ in water $(0.5 \mathrm{~mL})$ was added to a stirred solution of methyl 3-((2-((tert-butoxycarbonyl)amino)ethyl)carbamoyl)-5-((5-(2,8-dimethyl-5H-dibenzo[a,d] [7] annulen-5-yl)-2oxo-4-thioxo-3,4-dihydropyrimidin- $1(2 \mathrm{H})$-yl)methyl)benzoate $(0.1 \mathrm{~g}$, $0.15 \mathrm{mmol})$ in methanol $(1.5 \mathrm{~mL})$. After $16 \mathrm{~h}$, the mixture was partitioned between ethyl acetate and a $2 \mathrm{M}$ hydrochloric acid solution. The ethyl acetate solution was washed with brine, dried 
(magnesium sulfate), filtered, and evaporated in vacuo to give 3-((2((tert-butoxycarbonyl)amino)ethyl)carbamoyl)-5-((5-(2,8-dimethyl$5 H$-dibenzo $[a, d][7]$ annulen-5-yl)-2-oxo-4-thioxo-3,4-dihydropyrimidin-1 $(2 H)$-yl)methyl)benzoic acid $(0.08 \mathrm{~g})$ as a yellow solid. LC-MS (ESI+) Rt: 2.99 (254 nm, Method 1); $(\mathrm{m} / z)$ : $667.5(\mathrm{M}+1)$

PyBroP $(0.16 \mathrm{~g}, 0.34 \mathrm{mmol})$ was added to a stirred solution of 3-((2-((tert-butoxycarbonyl)amino)ethyl)carbamoyl)-5-((5-(2,8-dimethyl-5H-dibenzo[a,d][7] annulen-5-yl)-2-oxo-4-thioxo-3,4-dihydropyrimidin-1 $(2 \mathrm{H})$-yl)methyl)benzoic acid $(0.09 \mathrm{~g}, 0.135 \mathrm{mmol})$, 5-aminotetrazole monohydrate $(0.055 \mathrm{~g}, 0.54 \mathrm{mmol})$, and triethylamine $(0.13 \mathrm{~g}$, $1.3 \mathrm{mmol})$ in DMF $(2 \mathrm{~mL})$. After $0.5 \mathrm{~h}$, the solution was diluted with ethyl acetate $(15 \mathrm{~mL})$ and washed with $2 \mathrm{M}$ hydrochloric acid $(1 \times 4$ $\mathrm{mL})$, water $(2 \times 4 \mathrm{~mL})$, and brine $(1 \times 4 \mathrm{~mL})$. The solution was evaporated to dryness under reduced pressure. Purification was by silica-gel chromatography, and the eluent was methanol/dichloromethane, 5:95, and then $2 \%$ acetic acid in methanol/dichloromethane, 5:95. Yield: $0.03 \mathrm{~g}, 0.04 \mathrm{mmol}, 30 \%$ (yellow solid). ${ }^{1} \mathrm{H}$ NMR $(400 \mathrm{MHz}$, DMSO) $\delta: 12.66-12.59(\mathrm{~m}, 1 \mathrm{H}), 12.45-11.87(\mathrm{~m}, 1 \mathrm{H}), 8.83-8.67$ (m, $1 \mathrm{H}), 8.57-8.43(\mathrm{~m}, 1 \mathrm{H}), 8.09-7.94(\mathrm{~m}, 2 \mathrm{H}), 7.49-7.41(\mathrm{~m}, 2 \mathrm{H})$, 7.31-7.23 (m, 1H), 7.22-7.17 (m, 2H), 7.14-7.10 (m, 1H), 7.04$7.00(\mathrm{~m}, 1 \mathrm{H}), 6.99-6.89(\mathrm{~m}, 1 \mathrm{H}), 6.67-6.48(\mathrm{~m}, 2 \mathrm{H}), 5.75-5.69$ $(\mathrm{m}, 1 \mathrm{H}), 5.03-4.88(\mathrm{~m}, 2 \mathrm{H}), 3.44-3.33(\mathrm{~m}, 2 \mathrm{H}), 3.24-3.09(\mathrm{~m}, 2 \mathrm{H})$, 2.30-2.20 (m, 6H), 1.38 (s, 9H). LC-MS (ESI+) Rt: $2.98(254 \mathrm{~nm}$, Method 1); $(\mathrm{m} / \mathrm{z}): 734.3(\mathrm{M}+1)$.

tert-Butyl (2-(2-(2-(3-((2H-Tetrazol-5-yl)carbamoyl)-5-((5-(2,8-dimethyl-5H-dibenzo[a,d][7]annulen-5-yl)-2-oxo-4-thioxo-3,4-dihydropyrimidin-1(2H)-yl)methyl)benzamidolethoxy)ethoxy)ethyl)carbamate (65). Lithium hydroxide $(0.04 \mathrm{~g}, 1.6 \mathrm{mmol})$ in water $(0.5 \mathrm{~mL})$ was added to a stirred solution of methyl 3-((2,2-dimethyl-4oxo-3,8,11-trioxa-5-azatridecan-13-yl)carbamoyl)-5-((5-(2,8-dimethyl$5 H$-dibenzo[ $a, d][7]$ annulen-5-yl)-2-oxo-4-thioxo-3,4-dihydropyrimidin-1(2H)-yl)methyl)benzoate $(0.035 \mathrm{~g}, 0.046 \mathrm{mmol})$ in methanol $(1.5 \mathrm{~mL})$. After $16 \mathrm{~h}$, the mixture was partitioned between ethyl acetate and a $2 \mathrm{M}$ hydrochloric acid solution. The ethyl acetate solution was washed with brine, dried (magnesium sulfate), filtered, and evaporated in vacuo to give 3-((2,2-dimethyl-4-oxo-3,8,11-trioxa-5azatridecan-13-yl) carbamoyl)-5-((5-(2,8-dimethyl-5H-dibenzo[a,d][7] annulen-5-yl)-2-oxo-4-thioxo-3,4-dihydropyrimidin-1(2H)-yl)methyl)benzoic acid. Yield: $0.032 \mathrm{~g}$ (yellow solid). ${ }^{1} \mathrm{H}$ NMR LC-MS (ESI+) Rt: 3.04 (254 nm, Method 1); $(\mathrm{m} / z)$ : $755.6(\mathrm{M}+1)$. PyBroP $(0.042 \mathrm{~g}, 0.09 \mathrm{mmol})$ was added to a stirred solution of $3-((2,2-$ dimethyl-4-oxo-3,8,11-trioxa-5-azatridecan-13-yl) carbamoyl)-5-((5(2,8-dimethyl-5H-dibenzo[a,d][7] annulen-5-yl)-2-oxo-4-thioxo-3,4-dihydropyrimidin- $1(2 \mathrm{H})$-yl)methyl)benzoic acid $(0.05 \mathrm{~g}, 0.067 \mathrm{mmol})$, 5 -aminotetrazole monohydrate $(0.02 \mathrm{~g}, 0.2 \mathrm{mmol})$, and triethylamine $(0.03 \mathrm{~g}, 0.3 \mathrm{mmol})$ in DMF $(1 \mathrm{~mL})$. After $0.5 \mathrm{~h}$, the solution was diluted with ethyl acetate $(15 \mathrm{~mL})$ and washed with $2 \mathrm{M}$ hydrochloric acid $(1 \times 4 \mathrm{~mL})$, water $(2 \times 4 \mathrm{~mL})$, and brine $(1 \times 4 \mathrm{~mL})$. The solution was evaporate to dryness under reduced pressure. Purification was by silica-gel chromatography, and the eluent was methanol/dichloromethane, 5:95, and then $2 \%$ acetic acid in methanol/dichloromethane, 5:95. Yield: $0.009 \mathrm{~g}, 0.01 \mathrm{mmol}, 14 \%$ (yellow solid). ${ }^{1} \mathrm{H}$ NMR (400 MHz, DMSO) $\delta: 12.64(\mathrm{~s}, 1 \mathrm{H}), 12.51(\mathrm{~s}, 1 \mathrm{H}), 8.76(\mathrm{t}, \mathrm{J}=5.6 \mathrm{~Hz}$, $1 \mathrm{H}), 8.57(\mathrm{~s}, 1 \mathrm{H}), 8.03(\mathrm{~d}, 1 \mathrm{H}), 7.44(\mathrm{~d}, \mathrm{~J}=7.9 \mathrm{~Hz}, 2 \mathrm{H}), 7.12(\mathrm{dd}, \mathrm{J}=$ 8.1, $1.9 \mathrm{~Hz}, 2 \mathrm{H}), 7.00(\mathrm{~d}, \mathrm{~J}=1.9 \mathrm{~Hz}, 2 \mathrm{H}), 6.90(\mathrm{~s}, 1 \mathrm{H}), 6.79-6.73(\mathrm{~m}$, $1 \mathrm{H}), 6.54(\mathrm{~s}, 2 \mathrm{H}), 5.73(\mathrm{~s}, 1 \mathrm{H}), 4.97(\mathrm{~s}, 2 \mathrm{H}), 3.71-3.57(\mathrm{~m}, 4 \mathrm{H}), 3.54$ $(\mathrm{dd}, \mathrm{J}=6.1,4.1 \mathrm{~Hz}, 4 \mathrm{H}), 3.40(\mathrm{~d}, \mathrm{~J}=6.7 \mathrm{~Hz}, 2 \mathrm{H}), 3.07(\mathrm{q}, \mathrm{J}=6.0 \mathrm{~Hz}$, $2 \mathrm{H}), 2.22(\mathrm{~s}, 6 \mathrm{H}), 1.37(\mathrm{~s}, 9 \mathrm{H})$. LC-MS (ESI+) Rt: $3.02(254 \mathrm{~nm}$, Method 1); $(\mathrm{m} / z): 822.3(\mathrm{M}+1)$

(E)-N1-(2-(2-(4-(2-(5,5-Difluoro-7-(thiophen-2-yl)-5H-4 $\lambda^{4}, 5 \lambda^{4}-$ dipyrrolo[1,2-c:2' $\left.{ }^{\prime} 1^{\prime}-f\right][1,3,2]$ diazaborinin-3-yl)vinyl) phenoxy)acetamido)ethyl)-5-((5-(2,8-dimethyl-5H-dibenzo[a,d][7]annulen-5yl)-2-oxo-4-thioxo-3,4-dihydropyrimidin-1(2H)-yl)methyl)-N3-(1Htetrazol-5-yl)isophthalamide (66). Following general procedure 3 with tert-butyl (2-(3-((2H-tetrazol-5-yl) carbamoyl)-5-((5-(2,8-dimethyl-5H-dibenzo[ $a, d][7]$ annulen-5-yl)-2-oxo-4-thioxo-3,4-dihydropyrimidin-1(2H)-yl)methyl)benzamido)ethyl)carbamate (3.2 mg, $4.3 \times$ $\left.10^{-3} \mathrm{mmol}\right)$ and 2,5-dioxopyrrolidin-1-yl (E)-2-(4-(2-(5,5-difluoro-7(thiophen-2-yl)-5H-4 $\lambda^{4}, 5 \lambda^{4}$-dipyrrolo $\left[1,2-c: 2^{\prime}, 1^{\prime}-f\right][1,3,2]$ diazaborinin-3-yl)vinyl)phenoxy)acetate $\left(2.3 \mathrm{mg}, 4.2 \times 10^{-3} \mathrm{mmol}\right)$, the title compound was generated. Yield: $0.67 \mathrm{mg}, 0.627 \times 10^{-3} \mathrm{mmol}$, 15\%. LC-MS (ESI+) Rt: 3.19 (254 nm); $(\mathrm{m} / z): 1066.4(\mathrm{M}+1)$.

(E)-N1-(2-(6-(2-(4-(2-(5,5-Difluoro-7-(thiophen-2-yl)-5H-4 $\lambda^{4}, 5 \lambda^{4}$ dipyrrolo[1,2-c:2' $\left.1^{\prime}-f\right][1,3,2]$ diazaborinin-3-yl)vinyl)phenoxy)acetamido)hexanamido)ethyl)-5-((5-(2,8-dimethyl-5H-dibenzo-

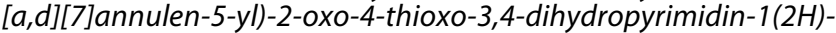
yl)methyl)-N3-(1H-tetrazol-5-yl)isophthalamide (67). Following general procedure 3 with tert-butyl (2-(3-( $(2 H$-tetrazol-5-yl)carbamoyl)-5( 5 -(2,8-dimethyl-5H-dibenzo[a,d][7] annulen-5-yl)-2-oxo-4-thioxo3,4-dihydropyrimidin-1 $(2 \mathrm{H})$-yl)methyl)benzamido)ethyl)carbamate $\left(2.2 \mathrm{mg}, 3 \times 10^{-3} \mathrm{mmol}\right)$ and 2,5-dioxopyrrolidin-1-yl (E)-6-(2-(4-(2(5,5-difluoro-7-(thiophen-2-yl)-5H-4 $\lambda^{4}, 5 \lambda^{4}$-dipyrrolo[1,2-c: $\left.2^{\prime}, 1^{\prime}-f\right]$ $[1,3,2]$ diazaborinin-3-yl)vinyl)phenoxy)acetamido)hexanoate $(2.0 \mathrm{mg}$, $\left.3 \times 10^{-3} \mathrm{mmol}\right)$, the title compound was generated. Yield: $1.2 \mathrm{mg}, 1 \times$ $10^{-3} \mathrm{mmol}, 45 \%$. LC-MS (ESI+) Rt: 7.35 (254 nm); $(\mathrm{m} / z): 1179.4$ $(\mathrm{M}+1)$.

(E)-N1-(2-(2-(2-(2-(4-(2-) 5,5-Difluoro-7-(thiophen-2-yl)-5H$4 \lambda^{4}, 5 \lambda^{4}$-dipyrrolo[1,2-c:2', $\left.1^{\prime}-f\right][1,3,2]$ diazaborinin-3-yl) vinyl)phenoxy)acetamido)ethoxy)ethoxy)ethyl)-5-((5-(2,8-dimethyl-5Hdibenzo[a,d][7]annulen-5-yl)-2-oxo-4-thioxo-3,4-dihydropyrimidin1(2H)-yl)methyl)-N3-(1H-tetrazol-5-yl) isophthalamide (68). Following general procedure 3 with tert-butyl (2-(2-(2- $(3-((2 H$-tetrazol-5yl) carbamoyl)-5-((5-(2,8-dimethyl-5H-dibenzo[a,d][7] annulen-5-yl)2-oxo-4-thioxo-3,4-dihydropyrimidin-1 $(2 \mathrm{H})$-yl)methyl)benzamido)ethoxy)ethoxy)ethyl)carbamate (3.4 $\left.\mathrm{mg}, 4.14 \times 10^{-3} \mathrm{mmol}\right)$ and 2,5 dioxopyrrolidin-1-yl (E)-2-(4-(2-(5,5-difluoro-7-(thiophen-2-yl)-5H$4 \lambda^{4}, 5 \lambda^{4}$-dipyrrolo[1,2-c:2', $\left.1^{\prime}-f\right][1,3,2]$ diazaborinin-3-yl)vinyl)phenoxy)acetate $\left(2.26 \mathrm{mg}, 4.14 \times 10^{-3} \mathrm{mmol}\right)$, the title compound was generated. Yield: $0.7 \mathrm{mg}, 0.67 \times 10^{-3} \mathrm{mmol}, 17 \%$. LC-MS (ESI+) Rt: $7.46(254 \mathrm{~nm}) ;(\mathrm{m} / z): 1154.6(\mathrm{M}+1)$.

(E)-N1-(18-(4-(2-(5,5-Difluoro-7-(thiophen-2-yl)-5H-4 $\lambda^{4}, 5 \lambda^{4}$ dipyrrolo[1,2-c:2', $\left.1^{\prime}-f\right][1,3,2]$ diazaborinin-3-yl) vinyl)phenoxy)10,17-dioxo-3,6-dioxa-9,16-diazaoctadecyl)-5-((5-(2,8-dimethyl5H-dibenzo[a,d][7]annulen-5-yl)-2-oxo-4-thioxo-3,4-dihydropyrimidin-1(2H)-yl)methyl)-N3-(1H-tetrazol-5-yl)isophthalamide (69). $\mathrm{HCl}$ $(4 \mathrm{M})$ in 1,4-dioxane $(1 \mathrm{~mL})$ was added to tert-butyl (2-(2-(2-(3( $(2 H$-tetrazol-5-yl)carbamoyl)-5-((5-(2,8-dimethyl-5H-dibenzo[ $a, d]$ [7] annulen-5-yl)-2-oxo-4-thioxo-3,4-dihydropyrimidin-1(2H)-yl)methyl)benzamido)ethoxy)ethoxy)ethyl)carbamate (1.5 mg, $1.82 \times$ $\left.10^{-3} \mathrm{mmol}\right)$. The solution was stirred for $2 \mathrm{~h}$ and then evaporated to dryness in vacuo. DMF $(0.5 \mathrm{~mL})$, then di-isopropylethylamine $(10 \mathrm{mg})$, and then 2,5-dioxopyrrolidin-1-yl (E)-6-(2-(4-(2-(5,5-difluoro-7-(thiophen-2-yl)-5H-4 $\lambda^{4}, 5 \lambda^{4}$-dipyrrolo[1,2-c: $\left.2^{\prime}, 1^{\prime}-f\right][1,3,2]$ diazaborinin-3yl)vinyl)phenoxy)acetamido)hexanoate $\left(1.2 \mathrm{mg}, 1.8 \times 10^{-3} \mathrm{mmol}\right)$ were added to the solution. The reaction mixture was stirred in the dark and under nitrogen overnight. Purification was by reverse-phase preparative HPLC eluted with a gradient of $50-60 \% \mathrm{MeCN}$ in water ( $0.1 \%$ formic acid) over $20 \mathrm{~min}$. The fraction containing the product was lyophilized. Yield: $1.002 \mathrm{mg}, 0.79 \times 10^{-3} \mathrm{mmol}, 41 \%$. LC-MS (ESI+) Rt: $7.41(254 \mathrm{~nm}) ;(m / z): 1267.0(\mathrm{M}+1)$.

tert-Butyl 3-((7-Chloro-4-(1-methyl-2,4-dioxo-1,2,3,4-tetrahydropyrimidin-5-yl)-4H-benzo[5,6]cyclohepta[1,2-d]thiazol-2-yl)amino)propanoate (70). Following general procedure 1, 43 (200 mg, $0.51 \mathrm{mmol}$ ) was reacted with $\beta$-alanine-tert-butyl ester hydrochloride (370 mg, $2.04 \mathrm{mmol}$ ) to afford the title compound, 70, which was isolated at $5 \% \mathrm{MeOH} / \mathrm{DCM}$ as a pale-yellow solid $(248 \mathrm{mg}, 0.494 \mathrm{mmol}$, 96\%). LC-MS (ESI+) Rt: $2.79 \min (254 \mathrm{~nm}$, Method 1); $(\mathrm{m} / z): 500.9$ $\left[\mathrm{M}\left({ }^{35} \mathrm{Cl}\right)+\mathrm{H}\right]^{+}$. ${ }^{1} \mathrm{H}$ NMR $\left(400 \mathrm{MHz}\right.$, DMSO- $\left.d_{6}\right) \delta: 11.20(\mathrm{~s}, 1 \mathrm{H})$, $7.97(\mathrm{t}, J=5.5 \mathrm{~Hz}, 1 \mathrm{H}), 7.46(\mathrm{~d}, J=8.4 \mathrm{~Hz}, 1 \mathrm{H}), 7.44(\mathrm{~d}, J=2.2 \mathrm{~Hz}$, $1 \mathrm{H}), 7.38(\mathrm{dd}, J=8.3,2.3 \mathrm{~Hz}, 1 \mathrm{H}), 7.03(\mathrm{~s}, 1 \mathrm{H}), 6.82(\mathrm{~d}, J=11.6 \mathrm{~Hz}$, $1 \mathrm{H}), 6.79(\mathrm{~d}, J=11.7 \mathrm{~Hz}, 1 \mathrm{H}), 5.22(\mathrm{~s}, 1 \mathrm{H}), 3.48-3.42(\mathrm{~m}, 2 \mathrm{H}), 3.17$ (s, 3H), 1.39 (s, 9H). ${ }^{13} \mathrm{C}$ NMR (101 MHz, DMSO-d 6 ) $\delta: 171.0$, 169.3 , 163.3, 151.1, 147.8, 143.1, 137.0, 134.2, 133.14, 133.00, 132.96, 131.0, 126.2, 122.2, 117.0, 108.9, 80.5, 43.8, 40.5, 36.2, 35.2, 28.2.

tert-Butyl 6-((7-Chloro-4-(1-methyl-2,4-dioxo-1,2,3,4-tetrahydropyrimidin-5-yl)-4H-benzo[5,6]cyclohepta[1,2-d]thiazol-2-yl)amino)hexanoate (71). Following general procedure 1, 43 (200 mg, $0.51 \mathrm{mmol})$ was reacted with tert-butyl 6-aminohexanoate $(382 \mathrm{mg}$, $2.04 \mathrm{mmol}$ ) to afford the title compound, 71 , at $5 \% \mathrm{MeOH} / \mathrm{DCM}$ as a pale-yellow solid (248 mg, $0.494 \mathrm{mmol}, 97 \%)$. LC-MS (ESI+) Rt: $2.91 \mathrm{~min}\left(254 \mathrm{~nm}\right.$, Method 1); $(\mathrm{m} / \mathrm{z}): 543.0\left[\mathrm{M}\left({ }^{35} \mathrm{Cl}\right)+\mathrm{H}\right]^{+} .{ }^{1} \mathrm{H}$ NMR 
(400 MHz, DMSO- $\left.d_{6}\right) \delta: 11.24(\mathrm{~s}, 1 \mathrm{H}), 7.92(\mathrm{t}, J=5.4 \mathrm{~Hz}, 1 \mathrm{H}), 7.45$ $(\mathrm{d}, J=8.4 \mathrm{~Hz}, 1 \mathrm{H}), 7.43(\mathrm{~d}, J=2.2 \mathrm{~Hz}, 1 \mathrm{H}), 7.37(\mathrm{dd}, J=8.3,2.2 \mathrm{~Hz}$, $1 \mathrm{H}), 7.01(\mathrm{~s}, 1 \mathrm{H}), 6.81(\mathrm{~d}, J=11.6 \mathrm{~Hz}, 1 \mathrm{H}), 6.76(\mathrm{~d}, J=11.7 \mathrm{~Hz}, 1 \mathrm{H})$, $5.23(\mathrm{~s}, 1 \mathrm{H}), 3.22-3.15(\mathrm{~m}, 2 \mathrm{H}), 3.17(\mathrm{~s}, 3 \mathrm{H}), 2.18(\mathrm{t}, J=7.3 \mathrm{~Hz}$, $2 \mathrm{H}), 1.56-1.49(\mathrm{~m}, 4 \mathrm{H}), 1.38(\mathrm{~s}, 9 \mathrm{H}), 1.34-1.30(\mathrm{~m}, 2 \mathrm{H}) .{ }^{13} \mathrm{C} \mathrm{NMR}$ $\left(101 \mathrm{MHz}, \mathrm{DMSO}-d_{6}\right) \delta: 172.7,169.8,163.3,151.1,148.0,142.9$, 137.1, 134.1, 133.1, 131.0, 129.3, 128.2, 125.9, 122.3, 116.6, 108.9, $79.8,44.6,43.9,36.2,35.2,28.7,28.2,26.3,24.8$.

3-((7-Chloro-4-(1-methyl-2-oxo-4-thioxo-1,2,3,4-tetrahydropyrimidin-5-yl)-4H-benzo[5,6]cyclohepta[1,2-d]thiazol-2-yl)amino)propanoic Acid (72). Following general procedure 2, 70 (200 mg, $0.40 \mathrm{mmol}$ ) was converted to the thiouracil, which was isolated at $2 \%$ $\mathrm{MeOH} / \mathrm{DCM}$ as a yellow solid $(124 \mathrm{mg})$. The intermediate product $(100 \mathrm{mg}, 0.193 \mathrm{mmol})$ was dissolved in DCM $(12 \mathrm{~mL})$, treated with trifluoroacetic acid $(6 \mathrm{~mL})$, and stirred at $\mathrm{rt}$ for $30 \mathrm{~min}$ until completion was observed by TLC. The RM was diluted with toluene $(25 \mathrm{~mL})$ and concentrated in vacuo to one-fifth the volume; this was repeated three times before the product was concentrated to dryness to afford a yellow solid. This was purified by FC and washed with 5\% $\mathrm{MeOH} / \mathrm{DCM}$, and the title compound, 72, was eluted with $20 \%$ $\mathrm{MeOH} / \mathrm{DCM}$ with $1 \%$ acetic acid as a yellow solid (34 mg, $0.074 \mathrm{mmol}, 39 \%)$. LC-MS (ESI+) Rt: $2.61 \mathrm{~min}(254 \mathrm{~nm}$, Method 1); $\left.(m / z): 460.9\left[\mathrm{M}\left({ }^{35} \mathrm{Cl}\right)+\mathrm{H}\right]\right]^{+} .{ }^{1} \mathrm{H}$ NMR $\left(400 \mathrm{MHz}\right.$, DMSO-d $\left.d_{6}\right) \delta$ : 12.66 (s, 1H), 12.45-12.07 (br.s, 1H), 7.99 (t, $J=5.2 \mathrm{~Hz}, 1 \mathrm{H}), 7.71$ $(\mathrm{s}, 1 \mathrm{H}), 7.55(\mathrm{~d}, J=8.5 \mathrm{~Hz}, 1 \mathrm{H}), 7.45(\mathrm{~d}, J=2.3 \mathrm{~Hz}, 1 \mathrm{H}), 7.35(\mathrm{dd}$, $J=8.4,2.3 \mathrm{~Hz}, 1 \mathrm{H}), 6.88(\mathrm{~s}, 2 \mathrm{H}), 5.68(\mathrm{~s}, 1 \mathrm{H}), 3.44($ app.q, $J=6.1 \mathrm{~Hz}$, $2 \mathrm{H}), 3.31(\mathrm{~s}, 3 \mathrm{H})$.

6-((7-Chloro-4-(1-methyl-2-oxo-4-thioxo-1,2,3,4-tetrahydropyrimidin-5-yl)-4H-benzo[5,6]cyclohepta[1,2-d]thiazol-2-yl)amino)hexanoic Acid (73). Following general procedure 2, 71 (200 mg, $0.40 \mathrm{mmol}$ ) was converted to the thiouracil, which was isolated at $2 \%$ $\mathrm{MeOH} / \mathrm{DCM}$ as a yellow solid $(124 \mathrm{mg})$. This was dissolved in DCM $(14 \mathrm{~mL})$, treated with trifluoroacetic acid $(7 \mathrm{~mL})$, and stirred at $\mathrm{rt}$ for 30 min until completion was observed by TLC. The RM was diluted with toluene $(25 \mathrm{~mL})$ and concentrated in vacuo to one-fifth the volume; this was repeated three times before the product was concentrated to dryness to afford a yellow solid. This was purified by FC and washed with $5 \% \mathrm{MeOH} / \mathrm{DCM}$, and the title compound, 73, was eluted with $20 \% \mathrm{MeOH} / \mathrm{DCM}$ with $1 \%$ acetic acid as a yellow solid (80 mg, $0.159 \mathrm{mmol}, 74 \%)$. LC-MS (ESI+) Rt: $2.67 \mathrm{~min}$ (254 nm, Method 1); $(m / z): 502.9\left[\mathrm{M}\left({ }^{35} \mathrm{Cl}\right)+\mathrm{H}\right]^{+} .{ }^{1} \mathrm{H}$ NMR (400 $\mathrm{MHz}$, DMSO-d $\left.d_{6}\right) \delta: 12.80-12.53$ (br.s, $1 \mathrm{H}$ ), 12.16-11.93 (br.s, $\left.1 \mathrm{H}\right), 7.94$ $(\mathrm{t}, J=5.3 \mathrm{~Hz}, 1 \mathrm{H}), 7.62(\mathrm{~s}, 1 \mathrm{H}), 7.58(\mathrm{~d}, J=8.5 \mathrm{~Hz}, 1 \mathrm{H}), 7.45(\mathrm{~d}, J=$ $2.3 \mathrm{~Hz}, 1 \mathrm{H}), 7.34(\mathrm{dd}, J=8.4,2.3 \mathrm{~Hz}, 1 \mathrm{H}), 6.86(\mathrm{~s}, 2 \mathrm{H}), 5.71(\mathrm{~s}, 1 \mathrm{H})$, $3.29(\mathrm{~s}, 3 \mathrm{H}), 3.23-3.18(\mathrm{~m}, 2 \mathrm{H}), 2.20(\mathrm{t}, J=7.3 \mathrm{~Hz}, 2 \mathrm{H}), 1.56-1.48$ (m, 4H), $1.36-1.29(\mathrm{~m}, 2 \mathrm{H}) .{ }^{13} \mathrm{C}$ NMR (101 MHz, DMSO-d $\left.d_{6}\right)$ $\delta: 189.2,174.9,169.6,149.1,148.5,143.3,137.6,134.0,132.0,130.8$, $129.0,127.8,126.58,126.56,122.7,117.5,116.4,45.3,44.7,37.2$, 34.1, 26.5, 24.7 .

tert-Butyl (2-(3-((7-Chloro-4-(1-methyl-2-oxo-4-thioxo-1,2,3,4tetrahydropyrimidin-5-yl)-4H-benzo[5,6]cyclohepta[1,2-d] thiazol2-yl)amino)propanamido)ethyl)carbamate (74). A stirred solution of $72(10 \mathrm{mg}, 0.022 \mathrm{mmol})$ in DMF $(1 \mathrm{~mL})$ was treated with DIPEA $(50 \mu \mathrm{L})$ and $N$-Boc-ethylenediamine $(21 \mathrm{mg}, 0.132 \mathrm{mmol})$ followed by PyBroP ( $15 \mathrm{mg}, 0.033 \mathrm{mmol}$ ) and stirred at rt for $1 \mathrm{~h}$ until completion was observed by LC-MS. The RM was quenched with water (ca. two drops), concentrated in vacuo, and azeotroped with toluene $(2 \times 25 \mathrm{~mL})$ to afford an orange oil. This was purified by FC $(1-5 \%$ $\mathrm{MeOH} / \mathrm{DCM}$ ) to afford the title compound, 74, at 4\% MeOH/DCM as a yellow solid (5.2 $\mathrm{mg}, 0.0086 \mathrm{mmol}, 39 \%)$. LC-MS (ESI+) Rt: $2.75 \min \left(254 \mathrm{~nm}\right.$, Method 1); $(\mathrm{m} / z): 603.1\left[\mathrm{M}\left({ }^{35} \mathrm{Cl}\right)+\mathrm{H}\right]^{+}$. ${ }^{1} \mathrm{H}$ NMR (400 MHz, DMSO- $\left.d_{6}\right) \delta: 12.67(\mathrm{~s}, 1 \mathrm{H}), 7.95(\mathrm{t}, J=5.5 \mathrm{~Hz}$, $1 \mathrm{H}), 7.91(\mathrm{t}, J=5.4 \mathrm{~Hz}, 1 \mathrm{H}), 7.74(\mathrm{~s}, 1 \mathrm{H}), 7.55(\mathrm{~d}, J=8.6 \mathrm{~Hz}, 1 \mathrm{H})$, $7.45(\mathrm{~d}, J=2.3 \mathrm{~Hz}, 1 \mathrm{H}), 7.35(\mathrm{dd}, J=8.4,2.3 \mathrm{~Hz}, 1 \mathrm{H}), 6.88(\mathrm{~s}, 2 \mathrm{H})$, $6.78(\mathrm{t}, J=5.3 \mathrm{~Hz}, 1 \mathrm{H}), 5.66(\mathrm{~s}, 1 \mathrm{H}), 3.45($ app.q, $J=6.5 \mathrm{~Hz}, 2 \mathrm{H})$, $3.32(\mathrm{~s}, 3 \mathrm{H}), 3.07-3.03(\mathrm{~m}, 2 \mathrm{H}), 2.96($ app.q, $J=6.1 \mathrm{~Hz}, 2 \mathrm{H}), 2.38$ $(\mathrm{t}, J=6.8 \mathrm{~Hz}, 2 \mathrm{H}), 1.38(\mathrm{~s}, 9 \mathrm{H})$.

tert-Butyl (2-(6-((7-Chloro-4-(1-methyl-2-oxo-4-thioxo-1,2,3,4tetrahydropyrimidin-5-yl)-4H-benzo[5,6]cyclohepta[1,2-d] thiazol2-yl)amino)hexanamido)ethyl)carbamate (75). A stirred solution of $73(30 \mathrm{mg}, 0.060 \mathrm{mmol})$ in DMF $(3 \mathrm{~mL})$ was treated with DIPEA
$(150 \mu \mathrm{L})$ and $N$-Boc-ethylenediamine $(58 \mathrm{mg}, 0.362 \mathrm{mmol})$ followed by PyBroP ( $42 \mathrm{mg}, 0.090 \mathrm{mmol}$ ) and stirred at rt for $1 \mathrm{~h}$ until completion was observed by LC-MS. The RM was quenched with water (ca. two drops), concentrated in vacuo, and azeotroped with toluene $(2 \times 25 \mathrm{~mL})$ to afford an orange oil. This was purified by FC $(1-5 \%$ $\mathrm{MeOH} / \mathrm{DCM}$ ) to afford the title compound, 75, at $4 \% \mathrm{MeOH} / \mathrm{DCM}$ (15 mg, $0.023 \mathrm{mmol}, 39 \%)$. LC-MS (ESI+) Rt: $2.80 \mathrm{~min}(254 \mathrm{~nm}$, Method 1); $\left.(\mathrm{m} / z): 645.1\left[\mathrm{M}\left({ }^{35} \mathrm{Cl}\right)+\mathrm{H}\right]\right]^{+} .{ }^{1} \mathrm{H}$ NMR $(400 \mathrm{MHz}$, DMSO- $\left.d_{6}\right) \delta: 12.66(\mathrm{~s}, 1 \mathrm{H}), 7.93(\mathrm{t}, J=5.4 \mathrm{~Hz}, 1 \mathrm{H}), 7.78-7.77$ $(\mathrm{m}, 1 \mathrm{H}), 7.62(\mathrm{~s}, 1 \mathrm{H}), 7.57(\mathrm{~d}, J=8.5 \mathrm{~Hz}, 1 \mathrm{H}), 7.45(\mathrm{~d}, J=2.3 \mathrm{~Hz}$, $1 \mathrm{H}), 7.34(\mathrm{dd}, J=8.4,2.3 \mathrm{~Hz}, 1 \mathrm{H}), 6.86(\mathrm{~s}, 2 \mathrm{H}), 6.77(\mathrm{t}, J=5.5 \mathrm{~Hz}$, $1 \mathrm{H}), 5.71(\mathrm{~s}, 1 \mathrm{H}), 3.29(\mathrm{~s}, 3 \mathrm{H}), 3.22-3.17(\mathrm{~m}, 2 \mathrm{H}), 3.05$ (app.q, $J=$ $5.8 \mathrm{~Hz}, 2 \mathrm{H}), 2.98-2.94(\mathrm{~m}, 2 \mathrm{H}), 2.04(\mathrm{t}, J=7.3 \mathrm{~Hz}, 2 \mathrm{H}), 1.55-1.47$ $(\mathrm{m}, 4 \mathrm{H}), 1.37(\mathrm{~s}, 9 \mathrm{H}), 1.31(\mathrm{t}, J=7.6 \mathrm{~Hz}, 2 \mathrm{H})$.

tert-Butyl (2-(2-(2-(3-)(7-Chloro-4-(1-methyl-2-oxo-4-thioxo1,2,3,4-tetrahydropyrimidin-5-yl)-4H-benzo[5,6]cyclohepta[1,2-d]thiazol-2-yl)amino)propanamido)ethoxy)ethoxy)ethyl)carbamate (76). A stirred solution of $72(10 \mathrm{mg}, 0.022 \mathrm{mmol})$ in DMF $(1 \mathrm{~mL})$ was treated with DIPEA $(50 \mu \mathrm{L})$ and $N$-Boc-2,2'-(ethylenedioxy)diethylamine ( $33 \mathrm{mg}, 0.132 \mathrm{mmol}$ ) followed by PyBroP (15 mg, $0.033 \mathrm{mmol}$ ) and stirred at $\mathrm{rt}$ for $1 \mathrm{~h}$ until completion was observed by LC-MS. The RM was quenched with water (ca. two drops), concentrated in vacuo, and azeotroped with toluene $(2 \times 25 \mathrm{~mL})$ to afford an orange oil. This was purified by FC $(1-5 \% \mathrm{MeOH} / \mathrm{DCM})$ to afford the title compound, 76 , at $4 \% \mathrm{MeOH} / \mathrm{DCM}$ as a yellow solid $(8.8 \mathrm{mg}$, $0.0127 \mathrm{mmol}, 58 \%)$. LC-MS (ESI+) Rt: $2.76 \mathrm{~min}(254 \mathrm{~nm}$, Method 1); $(m / z): 691.1\left[\mathrm{M}\left({ }^{35} \mathrm{Cl}\right)+\mathrm{H}\right]{ }^{+}$. ${ }^{1} \mathrm{H}$ NMR $\left(400 \mathrm{MHz}\right.$, DMSO- $\left.d_{6}\right)$ $\delta: 12.67(\mathrm{~s}, 1 \mathrm{H}), 7.95(\mathrm{t}, J=5.5 \mathrm{~Hz}, 2 \mathrm{H}), 7.79(\mathrm{~s}, 1 \mathrm{H}), 7.53(\mathrm{~d}, J=$ $8.4 \mathrm{~Hz}, 1 \mathrm{H}), 7.45(\mathrm{~d}, J=2.3 \mathrm{~Hz}, 1 \mathrm{H}), 7.34(\mathrm{dd}, J=8.4,2.3 \mathrm{~Hz}, 1 \mathrm{H})$, $6.88(\mathrm{~s}, 2 \mathrm{H}), 6.76(\mathrm{t}, J=5.4 \mathrm{~Hz}, 1 \mathrm{H}), 5.64(\mathrm{~s}, 1 \mathrm{H})$.

tert-Butyl (2-(2-(2-(6-)(7-Chloro-4-(1-methyl-2-oxo-4-thioxo1,2,3,4-tetrahydropyrimidin-5-yl)-4H-benzo[5,6]cyclohepta[1,2-d]thiazol-2-yl)amino)hexanamido)ethoxy)ethoxy)ethyl)carbamate (77). A stirred solution of $73(30 \mathrm{mg}, 0.060 \mathrm{mmol})$ in DMF $(3 \mathrm{~mL})$ was treated with DIPEA $(150 \mu \mathrm{L})$ and $N$-Boc-ethylenediamine $(58 \mathrm{mg}$, $0.362 \mathrm{mmol}$ ) followed by PyBroP ( $42 \mathrm{mg}, 0.090 \mathrm{mmol}$ ) and stirred at rt for $1 \mathrm{~h}$ until completion was observed by LC-MS. The RM was quenched with water (ca. two drops), concentrated in vacuo, and azeotroped with toluene $(2 \times 25 \mathrm{~mL})$ to afford an orange oil. This was purified by $\mathrm{FC}(1-5 \% \mathrm{MeOH} / \mathrm{DCM})$ to afford the title compound, 77, at $4 \% \mathrm{MeOH} / \mathrm{DCM}(16 \mathrm{mg}, 0.022 \mathrm{mmol}, 36 \%)$. LC-MS (ESI+) Rt: $2.81 \mathrm{~min}\left(254 \mathrm{~nm}\right.$, Method 1); $(\mathrm{m} / \mathrm{z}): 733.2\left[\mathrm{M}\left({ }^{35} \mathrm{Cl}\right)+\mathrm{H}\right]^{+}$. ${ }^{1} \mathrm{H}$ NMR (400 MHz, DMSO- $\left.d_{6}\right) \delta: 12.66(\mathrm{~s}, 1 \mathrm{H}), 7.93(\mathrm{t}, J=5.4 \mathrm{~Hz}$, $1 \mathrm{H}), 7.81(\mathrm{t}, J=5.6 \mathrm{~Hz}, 1 \mathrm{H}), 7.62(\mathrm{~s}, 1 \mathrm{H}), 7.57(\mathrm{~d}, J=8.5 \mathrm{~Hz}, 1 \mathrm{H})$, $7.44(\mathrm{~d}, J=2.3 \mathrm{~Hz}, 1 \mathrm{H}), 7.34(\mathrm{dd}, J=8.4,2.3 \mathrm{~Hz}, 1 \mathrm{H}), 6.86(\mathrm{~s}, 2 \mathrm{H})$, $6.76(\mathrm{t}, J=5.0 \mathrm{~Hz}, 1 \mathrm{H}), 5.71(\mathrm{~s}, 1 \mathrm{H}), 3.51-3.49(\mathrm{~m}, 2 \mathrm{H}), 3.38(\mathrm{q}, J=$ $5.3 \mathrm{~Hz}, 6 \mathrm{H}), 3.29(\mathrm{~s}, 3 \mathrm{H}), 3.22-3.16(\mathrm{~m}, 4 \mathrm{H}), 3.06(\mathrm{q}, J=6.0 \mathrm{~Hz}$, $2 \mathrm{H}), 2.06(\mathrm{t}, J=7.4 \mathrm{~Hz}, 2 \mathrm{H}), 1.57-1.52(\mathrm{~m}, 4 \mathrm{H}), 1.37(\mathrm{~s}, 9 \mathrm{H}), 1.33-$ $1.27(\mathrm{~m}, 2 \mathrm{H})$

(E)-3-((7-Chloro-4-(1-methyl-2-oxo-4-thioxo-1,2,3,4-tetrahydropyrimidin-5-yl)-4H-benzo[5,6]cyclohepta[1,2-d]thiazol-2-yl)amino)$N$-(2-(2-(4-(2-(5,5-difluoro-7-(thiophen-2-yl)-5H-4 $\lambda^{4}, 5 \lambda^{4}$-dipyrrolo$\left[1,2-c: 2^{\prime}, 1^{\prime}-f\right][1,3,2]$ diazaborinin-3-yl)vinyl)phenoxy)acetamido)ethyl)propanamide (78). Following general procedure 3, 74 was converted to the BODIPY630/650 conjugate, 78 . This was purified by preparative RP-HPLC (Method 4), which isolated 78 at a retention time of $13.45 \mathrm{~min}$. This compound was freeze-dried to a blue, iridescent solid (0.52 mg, $0.54 \mu \mathrm{mol}, 27 \%)$. LC-MS (ESI+) Rt: $3.04 \mathrm{~min}$ $\left(254 \mathrm{~nm}\right.$, Method 1); $(\mathrm{m} / z)$ : $935.1\left[\mathrm{M}\left({ }^{35} \mathrm{Cl}\right)+\mathrm{H}\right]^{+}$. H.MS-TOF (ESI+) $(m / z):[\mathrm{M}+\mathrm{H}]^{+}$calcd for $\mathrm{C}_{45} \mathrm{H}_{39} \mathrm{BClF}_{2} \mathrm{~N}_{8} \mathrm{O}_{4} \mathrm{~S}_{3}, 935.2001$; found, 935.2002. $[\mathrm{M}+\mathrm{Na}]^{+}$calcd for $\mathrm{C}_{45} \mathrm{H}_{38} \mathrm{BClF}_{2} \mathrm{~N}_{8} \mathrm{NaO}_{4} \mathrm{~S}_{3}$, 957.1820; found, 957.1838 .

(E)-N-(2-(3-((7-Chloro-4-(1-methyl-2-oxo-4-thioxo-1,2,3,4-tetrahydropyrimidin-5-yl)-4H-benzo[5,6]cyclohepta[1,2-d]thiazol-2-yl)amino)propanamido)ethyl)-6-(2-(4-(2-(5,5-difluoro-7-(thiophen-2yl)-5H-4 $\lambda^{4}, 5 \lambda^{4}$-dipyrrolo[1,2-c:2', $\left.1^{\prime}-f\right][1,3,2]$ diazaborinin-3-yl)vinyl)phenoxy)acetamido)hexanamide (79). Following general procedure 3, 74 was converted to the BODIPY630/650-X conjugate, 79. This was purified by preparative RP-HPLC (Method 4), which isolated 79 at a retention time of $13.37 \mathrm{~min}$. This compound was freeze-dried to a blue, iridescent solid $(0.85 \mathrm{mg}, 0.81 \mu \mathrm{mol}, 41 \%)$. LC-MS (ESI+) 
Rt: $3.01 \mathrm{~min}(254 \mathrm{~nm}$, Method 1$) ;(\mathrm{m} / z): 1048.2\left[\mathrm{M}\left({ }^{35} \mathrm{Cl}\right)+\mathrm{H}\right]^{+}$. H.MS-TOF (ESI-) $(\mathrm{m} / z):[\mathrm{M}-\mathrm{H}]^{-}$calcd for $\mathrm{C}_{51} \mathrm{H}_{48} \mathrm{BClF}_{2} \mathrm{~N}_{9} \mathrm{O}_{5} \mathrm{~S}_{3}$, 1046.2694; found, 1046.2657.

(E)-N-(2-(2-(2-(3-((7-Chloro-4-(1-methyl-2-oxo-4-thioxo-1,2,3,4tetrahydropyrimidin-5-yl)-4H-benzo[5,6]cyclohepta[1,2-d]thiazol2-yl)amino)propanamido)ethoxy)ethoxy)ethyl)-6-(2-(4-(2-(5,5-difluoro-7-(thiophen-2-yl)-5H-4 $\lambda^{4}, 5 \lambda^{4}$-dipyrrolo [1,2-c:2', $\left.1^{\prime}-f\right][1,3,2]$ diazaborinin-3-yl)vinyl)phenoxy)acetamido)hexanamide (80). Following general procedure 3, 76 was converted to the BODIPY630/ $650-\mathrm{X}$ conjugate, 80 . This was purified by preparative RP-HPLC (Method 4), which isolated 80 at a retention time of $13.03 \mathrm{~min}$. This compound was freeze-dried to a blue, iridescent solid $(0.57 \mathrm{mg}$, $0.50 \mu \mathrm{mol}, 25 \%)$. LC-MS (ESI+) Rt: $3.01 \mathrm{~min}(254 \mathrm{~nm}$, Method 1); $(m / z): 1136.2\left[\mathrm{M}\left({ }^{35} \mathrm{Cl}\right)+\mathrm{H}\right]^{+}$. H.MS-TOF (ESI-) $(\mathrm{m} / z):[\mathrm{M}-\mathrm{H}]^{-}$ calcd for $\mathrm{C}_{55} \mathrm{H}_{56} \mathrm{BClF}_{2} \mathrm{~N}_{9} \mathrm{O}_{7} \mathrm{~S}_{3}, 1134.3220$; found, 1134.3160 .

(E)-6-((7-Chloro-4-(1-methyl-2-oxo-4-thioxo-1,2,3,4-tetrahydropyrimidin-5-yl)-4H-benzo[5,6]cyclohepta[1,2-d]thiazol-2-yl)amino)$\mathrm{N}$-(2-(2-(4-(2-(5,5-difluoro-7-(thiophen-2-yl)-5H-5 $\lambda^{4}, 6 \lambda^{4}$-dipyrrolo$\left[1,2-c: 2^{\prime}, 1^{\prime}-f\right][1,3,2]$ diazaborinin-3-yl)vinyl)phenoxy)acetamido)ethyl)hexanamide (81). Following general procedure 3, 75 was converted to the BODIPY630/650 conjugate, 81 . This was purified by preparative RP-HPLC (Method 4), which isolated $\mathbf{8 1}$ at a retention time of $13.05 \mathrm{~min}$. This compound was freeze-dried to a blue, iridescent solid (0.41 mg, $0.42 \mu \mathrm{mol}, 21 \%)$. LC-MS (ESI+) Rt: $3.04 \mathrm{~min}$ $\left(254 \mathrm{~nm}\right.$, Method 1); $(\mathrm{m} / z): 977.2\left[\mathrm{M}\left({ }^{35} \mathrm{Cl}\right)+\mathrm{H}\right]^{+}$. H.MS-TOF (ESI+) $(m / z):[\mathrm{M}+\mathrm{H}]^{+}$calcd for $\mathrm{C}_{48} \mathrm{H}_{45} \mathrm{BClF}_{2} \mathrm{~N}_{8} \mathrm{O}_{4} \mathrm{~S}_{3}, 977.2470$; found, 977.2558. $[\mathrm{M}+\mathrm{Na}]^{+}$calcd for $\mathrm{C}_{48} \mathrm{H}_{44} \mathrm{BClF}_{2} \mathrm{~N}_{8} \mathrm{NaO}_{4} \mathrm{~S}_{3}$, 999.2289; found, 999.2380. H.MS-TOF (ESI-) $(\mathrm{m} / \mathrm{z}):[\mathrm{M}-\mathrm{H}]^{-}$calcd for $\mathrm{C}_{48} \mathrm{H}_{43} \mathrm{BClF}_{2} \mathrm{~N}_{8} \mathrm{O}_{4} \mathrm{~S}_{3}$, 975.2325; found, 975.2272.

(E)-6-((7-Chloro-4-(1-methyl-2-oxo-4-thioxo-1,2,3,4-tetrahydropyrimidin-5-yl)-4H-benzo[5,6]cyclohepta[1,2-d]thiazol-2-yl)amino)$N-\left(2-\left(2-\left(2-\left(2-\left(4-\left(2-\left(5,5-d i f l u o r o-7-(t h i o p h e n-2-y l)-5 H-4 \lambda^{4}, 5 \lambda^{4}-\right.\right.\right.\right.\right.\right.\right.$ dipyrrolo[1,2-c:2' $\left.{ }^{\prime} 1^{\prime}-f\right][1,3,2]$ diazaborinin-3-yl)vinyl)phenoxy)acetamido)ethoxy)ethoxy)ethyl)hexanamide (82). Following general procedure 3, 77 was converted to the BODIPY630/650 conjugate, 82. This was purified by preparative RP-HPLC (Method 4), which isolated 82 at a retention time of $13.69 \mathrm{~min}$. This compound was freezedried to a blue, iridescent solid $(0.22 \mathrm{mg}, 0.21 \mu \mathrm{mol}, 10 \%)$. LC-MS (ESI+) Rt: $3.05 \mathrm{~min}(254 \mathrm{~nm}$, Method 1); $(\mathrm{m} / \mathrm{z}): 1065.2$ $\left[\mathrm{M}\left({ }^{35} \mathrm{Cl}\right)+\mathrm{H}\right]^{+}$. H.MS-TOF (ESI-) $(\mathrm{m} / z):[\mathrm{M}-\mathrm{H}]^{-}$calcd for $\mathrm{C}_{52} \mathrm{H}_{51} \mathrm{BClF}_{2} \mathrm{~N}_{8} \mathrm{O}_{6} \mathrm{~S}_{3}, 1063.2849$; found, 1063.2803.

(E)-6-((7-Chloro-4-(1-methyl-2-oxo-4-thioxo-1,2,3,4-tetrahydropyrimidin-5-yl)-4H-benzo[5,6]cyclohepta[1,2-d]thiazol-2-yl)amino)$N-\left(2-\left(6-\left(2-\left(4-\left(2-\left(5,5-d i f l u o r o-7-(t h i o p h e n-2-y l)-5 H-4 \lambda^{4}, 5 \lambda^{4}\right.\right.\right.\right.\right.\right.$ dipyrrolo[1,2-c:2', $\left.1^{\prime}-f\right][1,3,2]$ diazaborinin-3-yl)vinyl)phenoxy)acetamido)hexanamido)ethyl)hexanamide (83). Following general procedure 3, 75 was converted to the BODIPY630/650-X conjugate, 83. This was purified by preparative RP-HPLC (Method 4), which isolated 83 at a retention time of $13.27 \mathrm{~min}$. This compound was freeze-dried to a blue, iridescent solid $(0.39 \mathrm{mg}, 0.36 \mu \mathrm{mol}, 18 \%)$. LC-MS (ESI+) Rt: $3.02 \mathrm{~min}(254 \mathrm{~nm}$, Method 1); $(\mathrm{m} / z)$ : 1090.2 $\left[\mathrm{M}\left({ }^{35} \mathrm{Cl}\right)+\mathrm{H}\right]^{+}$. H.MS-TOF (ESI+) $(\mathrm{m} / z):[\mathrm{M}+\mathrm{H}]^{+}$calcd for $\mathrm{C}_{54} \mathrm{H}_{56} \mathrm{BClF}_{2} \mathrm{~N}_{9} \mathrm{O}_{5} \mathrm{~S}_{3}, 1090.3311$; found, 1090.3293. $[\mathrm{M}+\mathrm{Na}]^{+}$calcd for $\mathrm{C}_{54} \mathrm{H}_{55} \mathrm{BClF}_{2} \mathrm{~N}_{9} \mathrm{NaO}_{5} \mathrm{~S}_{3}, 1112.3130$; found, 1112.3129.

(E)-6-((7-Chloro-4-(1-methyl-2-oxo-4-thioxo-1,2,3,4-tetrahydropyrimidin-5-yl)-4H-benzo[5,6]cyclohepta[1,2-d]thiazol-2-yl)amino)$N$-(18-(4-(2-(5,5-difluoro-7-(thiophen-2-yl)-5H-4 $\lambda^{4}, 5 \lambda^{4}$-dipyrrolo[1,2$\left.c: 2^{\prime}, 1^{\prime}-f\right][1,3,2]$ diazaborinin-3-yl)vinyl)phenoxy)-10,17-dioxo-3,6dioxa-9,16-diazaoctadecyl)hexanamide (84). Following general procedure 3, 77 was converted to the BODIPY630/650-X conjugate, 84. This was purified by preparative RP-HPLC (Method 4 ), which isolated 84 at a retention time of 13.28. This compound was freeze-dried to a blue, iridescent solid $(0.47 \mathrm{mg}, 0.40 \mu \mathrm{mol}, 20 \%)$. LC-MS (ESI+) Rt: $3.02 \mathrm{~min}\left(254 \mathrm{~nm}\right.$, Method 1); $(\mathrm{m} / z): 1178.2\left[\mathrm{M}\left({ }^{35} \mathrm{Cl}\right)+\mathrm{H}\right]^{+}$. H.MS-TOF (ESI+) $(m / z):[\mathrm{M}+\mathrm{H}]^{+}$calcd for $\mathrm{C}_{58} \mathrm{H}_{64} \mathrm{BClF}_{2} \mathrm{~N}_{9} \mathrm{O}_{7} \mathrm{~S}_{3}$, 1178.3835; found, 1178.3933. $[\mathrm{M}+\mathrm{Na}]^{+}$calcd for $\mathrm{C}_{58} \mathrm{H}_{63} \mathrm{BClF}_{2} \mathrm{~N}_{9^{-}}$ $\mathrm{NaO}_{7} \mathrm{~S}_{3}, 1200.3654$; found, 1200.3772 . H.MS-TOF (ESI-) $(\mathrm{m} / z)$ : $[\mathrm{M}-\mathrm{H}]^{-}$calcd for $\mathrm{C}_{58} \mathrm{H}_{62} \mathrm{BClF}_{2} \mathrm{~N}_{9} \mathrm{O}_{7} \mathrm{~S}_{3}, 1176.3689$; found, 1176.3647 .

3-((7-Chloro-4-(1-methyl-2-oxo-4-thioxo-1,2,3,4-tetrahydropyrimidin-5-yl)-4H-benzo[5,6]cyclohepta[1,2-d] thiazol-2-yl)amino)- $N$ (2-(3-(5,5-difluoro-7,9-dimethyl-5H-4 $\lambda^{4}, 5 \lambda^{4}$-dipyrrolo[1,2-c:2', $\left.1^{\prime}-f\right]$ [1,3,2]diazaborinin-3-yl)propanamido)ethyl)propanamide (85).
Following general procedure 3, 74 was converted to the BODIPYFL conjugate, $\mathbf{8 5}$. This was purified by preparative RP-HPLC (Method 3), which isolated 85 at a retention time of $13.82 \mathrm{~min}$. This compound was freeze-dried to a red-green, iridescent solid $(0.84 \mathrm{mg}, 1.08 \mu \mathrm{mol}$, $54 \%)$. LC-MS (ESI+) Rt: $2.80 \mathrm{~min}(254 \mathrm{~nm}$, Method 1); $(\mathrm{m} / z): 777.1$ $\left[\mathrm{M}\left({ }^{35} \mathrm{Cl}\right)+\mathrm{H}\right]^{+}$. H.MS-TOF $(\mathrm{ESI}+)(\mathrm{m} / z):[\mathrm{M}+\mathrm{H}]{ }^{+}$calcd for $\mathrm{C}_{36} \mathrm{H}_{37} \mathrm{BClF}_{2} \mathrm{~N}_{8} \mathrm{O}_{3} \mathrm{~S}_{2}$, 777.2174; found, 777.2106. $[\mathrm{M}+\mathrm{Na}]^{+}$calcd for $\mathrm{C}_{36} \mathrm{H}_{36} \mathrm{BClF}_{2} \mathrm{~N}_{8} \mathrm{NaO}_{3} \mathrm{~S}_{2}$, 799.1994; found, 799.1960. H.MS-TOF (ESI-) $(\mathrm{m} / z):[\mathrm{M}-\mathrm{H}]^{-}$calcd for $\mathrm{C}_{36} \mathrm{H}_{35} \mathrm{BClF}_{2} \mathrm{~N}_{8} \mathrm{O}_{3} \mathrm{~S}_{2}, 775.2029$; found, 775.2007 .

N-(2-(3-((7-Chloro-4-(1-methyl-2-oxo-4-thioxo-1,2,3,4-tetrahydropyrimidin-5-yl)-4H-benzo[5,6]cyclohepta[1,2-d]thiazol-2-yl)amino)propanamido)ethyl)-6-(3-(5,5-difluoro-7,9-dimethyl-5H$4 \lambda^{4}, 5 \lambda^{4}$-dipyrrolo $\left[1,2-c: 2^{\prime}, 1^{\prime}-f\right][1,3,2]$ diazaborinin-3-yl)propanamido)hexanamide (86). Following general procedure 3, 74 was converted to the BODIPYFL-X conjugate, 86. This was purified by preparative RP-HPLC (Method 3), which isolated 86 at a retention time of $14.37 \mathrm{~min}$. This compound was freeze-dried to a red-green, iridescent solid $(0.54 \mathrm{mg}, 0.61 \mu \mathrm{mol}, 30 \%)$. LC-MS (ESI+) Rt: $2.81 \mathrm{~min}\left(254 \mathrm{~nm}\right.$, Method 1); $(\mathrm{m} / z): 890.1\left[\mathrm{M}\left({ }^{35} \mathrm{Cl}\right)+\mathrm{H}\right]^{+}$. H.MSTOF (ESI+) $(m / z):[\mathrm{M}+\mathrm{H}]+$ calcd for $\mathrm{C}_{42} \mathrm{H}_{48} \mathrm{BClF}_{2} \mathrm{~N}{ }_{9} \mathrm{O}_{4} \mathrm{~S}_{2}$, 890.3015; found, 890.3014. $[\mathrm{M}+\mathrm{Na}]+$ calcd for $\mathrm{C}_{42} \mathrm{H}_{47} \mathrm{BClF}_{2} \mathrm{~N}$ ${ }_{9} \mathrm{NaO}_{4} \mathrm{~S}_{2}, 912.2834$; found, 912.2862. H.MS-TOF (ESI-) $(\mathrm{m} / z)$ : $[\mathrm{M}-\mathrm{H}]^{-}$calcd for $\mathrm{C}_{42} \mathrm{H}_{46} \mathrm{BClF}_{2} \mathrm{~N}_{9} \mathrm{O}_{4} \mathrm{~S}_{2}, 888.2869$; found, 888.2853. N-(2-(2-(2-(3-)(7-Chloro-4-(1-methyl-2-oxo-4-thioxo-1,2,3,4-tetrahydropyrimidin-5-yl)-4H-benzo[5,6]cyclohepta[1,2-d]thiazol-2yl)amino)propanamido)ethoxy)ethoxy)ethyl)-6-(3-(5,5-difluoro-7,9dimethyl-5H-4 $\lambda^{4}, 5 \lambda^{4}$-dipyrrolo $\left[1,2-c: 2^{\prime}, 1^{\prime}-f\right][1,3,2]$ diazaborinin-3yl)propanamido)hexanamide (87). Following general procedure 3, 76 was converted to the BODIPYFL-X conjugate, 87. This was purified by preparative RP-HPLC (Method 3), which isolated 87 at a retention time of $14.47 \mathrm{~min}$. This compound was freeze-dried to a redgreen, iridescent solid ( $0.46 \mathrm{mg}, 0.47 \mu \mathrm{mol}, 24 \%)$. LC-MS (ESI+) Rt: $2.78 \mathrm{~min}\left(254 \mathrm{~nm}\right.$, Method 1); $(\mathrm{m} / z): 978.3\left[\mathrm{M}\left({ }^{35} \mathrm{Cl}\right)+\mathrm{H}\right]^{+}$. H.MS-TOF (ESI+) $(m / z):[\mathrm{M}+\mathrm{Na}]^{+}$calcd for $\mathrm{C}_{46} \mathrm{H}_{55} \mathrm{BClF}_{2} \mathrm{~N}$ ${ }_{9} \mathrm{NaO}_{6} \mathrm{~S}_{2}, 1000.3359$; found, 1000.3457. H.MS-TOF (ESI-) $(\mathrm{m} / z)$ : $[\mathrm{M}-\mathrm{H}]^{-}$calcd for $\mathrm{C}_{46} \mathrm{H}_{54} \mathrm{BClF}_{2} \mathrm{~N}_{9} \mathrm{O}_{6} \mathrm{~S}_{2}, 976.3394$; found, 976.3355.

6-((7-Chloro-4-(1-methyl-2-oxo-4-thioxo-1,2,3,4-tetrahydropyrimidin-5-yl)-4H-benzo[5,6]cyclohepta[1,2-d]thiazol-2-yl)amino)- $N$ (2-(3-(5,5-difluoro-7,9-dimethyl-5H-5 $\lambda^{4}, 6 \lambda^{4}$-dipyrrolo[1,2-c:2', $\left.{ }^{\prime}-f\right]-$ $[1,3,2]$ diazaborinin-3-yl)propanamido)ethyl)hexanamide (88). Following general procedure 3,75 was converted to the BODIPYFL conjugate, $\mathbf{8 8}$. This was purified by preparative RP-HPLC (Method 3), which isolated 88 at a retention time of $15.19 \mathrm{~min}$. This compound was freeze-dried to a red-green, iridescent solid $(0.71 \mathrm{mg}, 0.87 \mu \mathrm{mol}$, 43\%). LC-MS (ESI+) Rt: $2.82 \mathrm{~min}(254 \mathrm{~nm}$, Method 1); $(\mathrm{m} / z): 819.2$ $\left[\mathrm{M}\left({ }^{35} \mathrm{Cl}\right)+\mathrm{H}\right]^{+}$. H.MS-TOF (ESI+) $(\mathrm{m} / z):[\mathrm{M}+\mathrm{H}]{ }^{+}$calcd for $\mathrm{C}_{39} \mathrm{H}_{43} \mathrm{BClF}_{2} \mathrm{~N}_{8} \mathrm{O}_{3} \mathrm{~S}_{2}, 819.2644$; found, 819.2598. $[\mathrm{M}+\mathrm{Na}]^{+}$calcd for $\mathrm{C}_{39} \mathrm{H}_{42} \mathrm{BClF}_{2} \mathrm{~N}_{8} \mathrm{NaO}_{3} \mathrm{~S}_{2}, 841.2463$; found, 841.2419.

6-((7-Chloro-4-(1-methyl-2-oxo-4-thioxo-1,2,3,4-tetrahydropyrimidin-5-yl)-4H-benzo[5,6]cyclohepta[1,2-d] thiazol-2-yl)amino)- $N$ (2-(2-(2-(3-(5,5-difluoro-7,9-dimethyl-5H-5 $\lambda^{4}, 6 \lambda^{4}$-dipyrrolo[1,2$\left.c: 2^{\prime}, 1^{\prime}-f\right][1,3,2]$ diazaborinin-3-yl)propanamido)ethoxy)ethoxy)ethyl)hexanamide (89). Following general procedure 3, 77 was converted to the BODIPYFL conjugate, 89 . This was purified by preparative RP-HPLC (Method 3), which isolated 89 at a retention time of $15.97 \mathrm{~min}$. This compound was freeze-dried to a red-green, iridescent solid (0.41 mg, $0.45 \mu \mathrm{mol}, 23 \%)$. LC-MS (ESI+) Rt: $2.83 \mathrm{~min}(254 \mathrm{~nm}$, Method 1); $(\mathrm{m} / z): 907.2\left[\mathrm{M}\left({ }^{35} \mathrm{Cl}\right)+\mathrm{H}\right]^{+}$. H.MS-TOF $(\mathrm{ESI}+)(\mathrm{m} / \mathrm{z})$ : $[\mathrm{M}+\mathrm{H}]^{+}$calcd for $\mathrm{C}_{43} \mathrm{H}_{51} \mathrm{BClF}_{2} \mathrm{~N}_{8} \mathrm{O}_{5} \mathrm{~S}_{2}, 907.3168$; found, 907.3160. $[\mathrm{M}+\mathrm{Na}]^{+}$calcd for $\mathrm{C}_{43} \mathrm{H}_{50} \mathrm{BClF}_{2} \mathrm{~N}_{8} \mathrm{NaO}_{5} \mathrm{~S}_{2}, 929.2987$; found, 929.2989. 6-((7-Chloro-4-(1-methyl-2-oxo-4-thioxo-1,2,3,4-tetrahydropyrimidin-5-yl)-4H-benzo[5,6]cyclohepta[1,2-d]thiazol-2-yl)amino)- $N$ (2-(6-(3-(5,5-difluoro-7,9-dimethyl-5H-5 $\lambda^{4}, 6 \lambda^{4}$-dipyrrolo[1,2-c:2', $1^{\prime}$ f] $[1,3,2]$ diazaborinin-3-yl)propanamido)hexanamido)ethyl)hexanamide (90). Following general procedure 3, 75 was converted to the BODIPYFL-X conjugate, 90. This was purified by preparative RP-HPLC (Method 3), which isolated 90 at a retention time of $15.59 \mathrm{~min}$. This compound was freeze-dried to a red-green, iridescent solid (0.43 mg, $0.46 \mu \mathrm{mol}, 23 \%)$. LC-MS (ESI+) Rt: $2.79 \mathrm{~min}(254 \mathrm{~nm}$, Method 1); $(\mathrm{m} / z)$ : $932.3\left[\mathrm{M}\left({ }^{35} \mathrm{Cl}\right)+\mathrm{H}\right]^{+}$. H.MS-TOF $(\mathrm{ESI}+)(\mathrm{m} / z)$ : $[\mathrm{M}+\mathrm{H}]^{+}$calcd for $\mathrm{C}_{45} \mathrm{H}_{54} \mathrm{BClF}_{2} \mathrm{~N}_{9} \mathrm{O}_{4} \mathrm{~S}_{2}, 932.3484$; found, 932.3534 . 
$[\mathrm{M}+\mathrm{Na}]+{ }^{+}$calcd for $\mathrm{C}_{45} \mathrm{H}_{53} \mathrm{BClF}_{2} \mathrm{~N}{ }_{9} \mathrm{NaO}_{4} \mathrm{~S}_{2}, 954.3304$; found, 954.3387.

6-((7-Chloro-4-(1-methyl-2-oxo-4-thioxo-1,2,3,4-tetrahydropyrimidin-5-yl)-4H-benzo[5,6]cyclohepta[1,2-d] thiazol-2-yl)amino)- $N$ (19-(5,5-difluoro-7,9-dimethyl-5H-5 $\lambda^{4}, 6 \lambda^{4}$-dipyrrolo $\left[1,2-c: 2^{\prime}, 1^{\prime}-f\right]$ [1,3,2]diazaborinin-3-yl)-10,17-dioxo-3,6-dioxa-9,16diazanonadecyl)hexanamide (91). Following general procedure 3 , 77 was converted to the BODIPYFL-X conjugate, 91. This was purified by preparative RP-HPLC (Method 3), which isolated 91 at a retention time of $15.92 \mathrm{~min}$. This compound was freeze-dried to a redgreen, iridescent solid $(0.45 \mathrm{mg}, 0.44 \mu \mathrm{mol}, 22 \%)$. LC-MS (ESI+) Rt: $2.80 \mathrm{~min}(254 \mathrm{~nm}$, Method 1$) ;(\mathrm{m} / z): 1020.4\left[\mathrm{M}\left({ }^{35} \mathrm{Cl}\right)+\mathrm{H}\right]^{+}$. H.MSTOF $($ ESI +$)(m / z):[\mathrm{M}+\mathrm{H}]{ }^{+}$calcd for $\mathrm{C}_{49} \mathrm{H}_{62} \mathrm{BClF}_{2} \mathrm{~N}_{9} \mathrm{O}_{6} \mathrm{~S}_{2}$, 1020.4009; found, 1020.4011. $[\mathrm{M}+\mathrm{Na}]^{+}$calcd for $\mathrm{C}_{49} \mathrm{H}_{61} \mathrm{BClF}_{2} \mathrm{~N}$ ${ }_{9} \mathrm{NaO}_{6} \mathrm{~S}_{22}, 1042.3828$; found, 1042.3837 .

Ethyl 2-((5-(2-((3-(tert-Butoxy)-3-oxopropyl)amino)-7-chloro-4Hbenzo[5,6]cyclohepta[1,2-d]thiazol-4-yl)-2-oxo-4-thioxo-3,4-dihydropyrimidin-1(2H)-yl)methyl)thiazole-4-carboxylate (92). Following general procedure $1,57(547 \mathrm{mg}, 1.00 \mathrm{mmol})$ was reacted with $\beta$-alanine-tert-butyl ester hydrochloride $(727 \mathrm{mg}, 4.00 \mathrm{mmol})$ to afford the title compound, 92, at $3 \% \mathrm{MeOH} / \mathrm{DCM}$ as a pale-yellow solid (585 mg, $0.89 \mathrm{mmol}, 89 \%)$. LC-MS (ESI+) Rt: $2.97 \mathrm{~min}(254 \mathrm{~nm}$, Method 1); $(\mathrm{m} / \mathrm{z}): 656.0\left[\mathrm{M}\left({ }^{35} \mathrm{Cl}\right)+\mathrm{H}\right]^{+}$. The product was reacted following general procedure 2 , and the intermediate uracil $(492 \mathrm{mg}$, $0.75 \mathrm{mmol}$ ) was converted to the title compound, 92, which was isolated at $1.5 \% \mathrm{MeOH} / \mathrm{DCM}$ as a yellow solid $(240 \mathrm{mg}, 0.36 \mathrm{mmol}$, 48\%). LC-MS (ESI+) Rt: $3.13 \mathrm{~min}(254 \mathrm{~nm}$, Method 1); $(\mathrm{m} / \mathrm{z}): 672.0$ $\left.\left[\mathrm{M}\left({ }^{35} \mathrm{Cl}\right)+\mathrm{H}\right]\right]^{+} .{ }^{1} \mathrm{H}$ NMR $\left(400 \mathrm{MHz}\right.$, DMSO- $\left.d_{6}\right) \delta: 12.88(\mathrm{~s}, 1 \mathrm{H})$, $8.56(\mathrm{~s}, 1 \mathrm{H}), 7.97(\mathrm{t}, J=5.5 \mathrm{~Hz}, 1 \mathrm{H}), 7.79(\mathrm{~s}, 1 \mathrm{H}), 7.57(\mathrm{~d}, J=8.5 \mathrm{~Hz}$, $1 \mathrm{H}), 7.41(\mathrm{~d}, J=2.3 \mathrm{~Hz}, 1 \mathrm{H}), 7.36(\mathrm{dd}, J=8.3,2.3 \mathrm{~Hz}, 1 \mathrm{H}), 6.77$ $(\mathrm{s}, 2 \mathrm{H}), 5.67(\mathrm{~s}, 1 \mathrm{H}), 5.40(\mathrm{~d}, J=15.8 \mathrm{~Hz}, 1 \mathrm{H}), 5.27(\mathrm{~d}, J=15.8 \mathrm{~Hz}$, $1 \mathrm{H}), 4.35$ (q, $J=6.9 \mathrm{~Hz}, 2 \mathrm{H}), 3.42$ (app.q, $J=6.2 \mathrm{~Hz}, 2 \mathrm{H}), 2.46(\mathrm{t}, J=$ $6.4 \mathrm{~Hz}, 2 \mathrm{H}), 1.35(\mathrm{~s}, 9 \mathrm{H}), 1.34(\mathrm{t}, J=6.9 \mathrm{~Hz}, 3 \mathrm{H}) .{ }^{13} \mathrm{C}$ NMR $\left(101 \mathrm{MHz}, \mathrm{DMSO}-d_{6}\right) \delta$ : $189.8,171.1,169.1,165.4,161.0,148.0$, $146.4,137.5,133.8,130.99,130.97,130.83,128.9,127.9,126.8,122.7$, $117.7,116.8,110.0,80.4,61.4,49.3,45.3,35.2,28.2,22.5,14.4$.

Ethyl 2-((5-(2-((3-)(2-)(tert-Butoxycarbonyl)amino)ethyl)amino)3-oxopropyl)amino)-7-chloro-4H-benzo[5,6]cyclohepta[1,2-d]thiazol-4-yl)-2-oxo-4-thioxo-3,4-dihydropyrimidin-1(2H)-yl)methyl)thiazole-4-carboxylate (93). A stirred solution of $92(200 \mathrm{mg}, 0.30$ $\mathrm{mmol})$ in DCM $(12 \mathrm{~mL})$ was treated with trifluoroacetic acid $(6 \mathrm{~mL})$ and stirred at rt for 30 min until completion was observed by TLC. The RM was diluted with toluene $(25 \mathrm{~mL})$ and concentrated in vacuo to one-fifth the volume; this was repeated three times before the product was concentrated to dryness to afford a yellow solid. LC-MS (ESI+) Rt: $2.77 \mathrm{~min}$ (254 nm, Method 1); $(\mathrm{m} / \mathrm{z}): 616.2$ $\left[\mathrm{M}\left({ }^{35} \mathrm{Cl}\right)+\mathrm{H}\right]^{+}$. The intermediate $(45 \mathrm{mg}, 0.073 \mathrm{mmol})$ in DCM $(3 \mathrm{~mL})$ was treated with $\mathrm{Et}_{3} \mathrm{~N}(60 \mu \mathrm{L})$ and $N$-Boc-ethylenediamine (70 $\mathrm{mg}, 0.438 \mathrm{mmol}$ ) followed by HATU $(56 \mathrm{mg}, 0.146 \mathrm{mmol})$ and stirred at rt for $24 \mathrm{~h}$ until completion was observed by LC-MS. The $\mathrm{RM}$ was quenched with $\mathrm{MeOH}(1 \mathrm{~mL})$ and concentrated directly onto silica. This was purified by FC $(1-5 \% \mathrm{MeOH} / \mathrm{DCM})$ to afford the title compound, 93 , at $5 \% \mathrm{MeOH} / \mathrm{DCM}$ as a yellow solid $(35 \mathrm{mg}$, $0.046 \mathrm{mmol}, 63 \%)$. LC-MS (ESI+) Rt: $2.88 \mathrm{~min}(254 \mathrm{~nm}$, Method 1); $\left.(m / z): 758.2\left[\mathrm{M}\left({ }^{35} \mathrm{Cl}\right)+\mathrm{H}\right]\right]^{+} .{ }^{1} \mathrm{H}$ NMR $\left(400 \mathrm{MHz}\right.$, DMSO- $\left.d_{6}\right)$ $\delta: 12.86(\mathrm{~s}, 1 \mathrm{H}), 8.55(\mathrm{~s}, 1 \mathrm{H}), 7.91(\mathrm{t}, J=5.7 \mathrm{~Hz}, 1 \mathrm{H}), 7.88(\mathrm{t}, J=$ $6.3 \mathrm{~Hz}, 1 \mathrm{H}), 7.75(\mathrm{~s}, 1 \mathrm{H}), 7.58(\mathrm{~d}, J=8.5 \mathrm{~Hz}, 1 \mathrm{H}), 7.40(\mathrm{~d}, J=2.2 \mathrm{~Hz}$, $1 \mathrm{H}), 7.36(\mathrm{dd}, J=8.3,2.3 \mathrm{~Hz}, 1 \mathrm{H}), 6.78-6.75(\mathrm{~m}, 1 \mathrm{H}), 6.75(\mathrm{~s}, 2 \mathrm{H})$, $5.68(\mathrm{~s}, 1 \mathrm{H}), 5.41(\mathrm{~d}, J=15.8 \mathrm{~Hz}, 1 \mathrm{H}), 5.26(\mathrm{~d}, J=15.8 \mathrm{~Hz}, 1 \mathrm{H}), 4.35$ (q, $J=7.0 \mathrm{~Hz}, 2 \mathrm{H}), 3.42$ (app.q, $J=6.2 \mathrm{~Hz}, 2 \mathrm{H}), 3.04$ (app.q, $J=6.0 \mathrm{~Hz}$, $2 \mathrm{H}), 2.97-2.93(\mathrm{~m}, 2 \mathrm{H}), 2.34(\mathrm{t}, J=6.7 \mathrm{~Hz}, 3 \mathrm{H}), 1.39-1.36(\mathrm{~m}, 9 \mathrm{H})$, $1.34(\mathrm{t}, J=7.1 \mathrm{~Hz}, 3 \mathrm{H})$.

tert-Butyl (2-(3-((4-(1-((4-((1H-Tetrazol-5-yl)carbamoyl)thiazol-2yl)methyl)-2-oxo-4-thioxo-1,2,3,4-tetrahydropyrimidin-5-yl)-7chloro-4H-benzo[5,6]cyclohepta[1,2-d]thiazol-2-yl)amino)propanamido)ethyl)carbamate (94). A stirred solution of $93(30 \mathrm{mg}$, $0.040 \mathrm{mmol})$ in $\mathrm{MeOH}(4 \mathrm{~mL})$ was treated with $1 \mathrm{M} \mathrm{NaOH}(120 \mu \mathrm{L}$, $0.120 \mathrm{mmol}$ ) and heated to reflux for $4 \mathrm{~h}$ under $\mathrm{N}_{2}$ until completion was observed by LC-MS. This was cooled, treated with $1 \mathrm{M} \mathrm{HCl}$ $(120 \mu \mathrm{L}, 0.120 \mathrm{mmol})$, diluted with toluene $(25 \mathrm{~mL})$, and concentrated in vacuo to one-fifth the volume; this was repeated three times before the product was concentrated to dryness to afford a yellow solid. This was dissolved in DMF $(2 \mathrm{~mL})$, treated with DIPEA $(35 \mu \mathrm{L})$ and 5 -aminotetrazole monohydrate $(25 \mathrm{mg}, 0.240 \mathrm{mmol})$ followed by PyBroP $(28 \mathrm{mg}, 0.60 \mathrm{mmol})$, and stirred at $\mathrm{rt}$ for $4 \mathrm{~h}$ until completion was observed by LC-MS. The RM was quenched with water (ca. two drops) and concentrated in vacuo. The DMF was azeotroped with toluene $(3 \times 50 \mathrm{~mL})$ to afford an orange oil. This was purified by FC and washed with $10 \% \mathrm{MeOH} / \mathrm{DCM}$ before the title compound, 94, was eluted with $10 \% \mathrm{MeOH} / \mathrm{DCM}$ with $1 \%$ acetic acid $(9 \mathrm{mg}$, $0.011 \mathrm{mmol}, 28 \%)$. LC-MS (ESI+) Rt: $2.68 \mathrm{~min}(254 \mathrm{~nm}$, Method 1); $(\mathrm{m} / z): 797.4\left[\mathrm{M}\left({ }^{35} \mathrm{Cl}\right)+\mathrm{H}\right]^{+}$. H.MS-TOF (ESI-) $(\mathrm{m} / z):[\mathrm{M}-\mathrm{H}]^{-}$ calcd for $\mathrm{C}_{32} \mathrm{H}_{32} \mathrm{ClN}_{12} \mathrm{O}_{5} \mathrm{~S}_{3}, 795.1475$; found, 795.1437. ${ }^{1} \mathrm{H}$ NMR $\left(500 \mathrm{MHz}, \mathrm{DMSO}-d_{6}\right) \delta$ : 12.92-12.78 (br.s, $\left.1 \mathrm{H}\right), 8.53-8.43$ (br.s, $1 \mathrm{H}), 7.99-7.89(\mathrm{~s}, 2 \mathrm{H}), 7.74-7.68$ (br.s, $1 \mathrm{H}), 7.58(\mathrm{~d}, J=8.4 \mathrm{~Hz}$, $1 \mathrm{H}), 7.40(\mathrm{~d}, J=2.2 \mathrm{~Hz}, 1 \mathrm{H}), 7.35$ (dd, $J=8.4,2.2 \mathrm{~Hz}, 1 \mathrm{H}), 6.78-$ $6.76(\mathrm{~m}, 2 \mathrm{H}), 5.68(\mathrm{~s}, 1 \mathrm{H}), 5.40(\mathrm{~d}, J=15.9 \mathrm{~Hz}, 1 \mathrm{H}), 5.29(\mathrm{~d}, J=$ $15.9 \mathrm{~Hz}, 1 \mathrm{H}), 4.12-4.09(\mathrm{~m}, 2 \mathrm{H}), 3.41$ (app.q, $J=6.5 \mathrm{~Hz}, 2 \mathrm{H}), 3.06-$ $3.03(\mathrm{~m}, 2 \mathrm{H}), 2.99-2.94(\mathrm{~m}, 2 \mathrm{H}), 1.36-1.21(\mathrm{~m}, 9 \mathrm{H})$.

(E)-2-((5-(7-Chloro-2-)(3-)(2-(2-)-(2-(5,5-difluoro-7-(thiophen-2yl)-5H-4 $\lambda^{4}, 5 \lambda^{4}$-dipyrrolo[1,2-c:2', $\left.1^{\prime}-f\right][1,3,2]$ diazaborinin-3-yl)vinyl)phenoxy)acetamido)ethyl)amino)-3-oxopropyl)amino)-4H-benzo[5,6]cyclohepta[1,2-d]thiazol-4-yl)-2-oxo-4-thioxo-3,4-dihydropyrimidin-1(2H)-yl)methyl)- $\mathrm{N}$-(1H-tetrazol-5-yl)thiazole-4-carboxamide (95). Following general procedure 3, 94 was converted to the BODIPY630/650 conjugate, 95. This was purified by preparative RP-HPLC (Method 4), which isolated 95 at a retention time of $10.80 \mathrm{~min}$. This compound was freeze-dried to a blue, iridescent solid $(0.35 \mathrm{mg}, 0.31 \mu \mathrm{mol}, 16 \%)$. LC-MS (ESI+) Rt: $3.00 \mathrm{~min}(254 \mathrm{~nm}$, Method 1); $(\mathrm{m} / z): 1129.5\left[\mathrm{M}\left({ }^{35} \mathrm{Cl}\right)+\mathrm{H}\right]^{+}$. H.MS-TOF (ESI +$)(\mathrm{m} / z)$ : $[\mathrm{M}+\mathrm{H}]^{+}$calcd for $\mathrm{C}_{50} \mathrm{H}_{41} \mathrm{BClF}_{2} \mathrm{~N}_{14} \mathrm{O}_{5} \mathrm{~S}_{4}, 1129.2011$; found, 1129.2000. $[\mathrm{M}+\mathrm{Na}]^{+}$calcd for $\mathrm{C}_{50} \mathrm{H}_{40} \mathrm{BClF}_{2} \mathrm{~N}_{14} \mathrm{NaO}_{5} \mathrm{~S}_{4}, 1151.1831$; found, 1151.1823. H.MS-TOF (ESI-) $(\mathrm{m} / z):[\mathrm{M}-\mathrm{H}]^{-}$calcd for $\mathrm{C}_{50} \mathrm{H}_{39} \mathrm{BClF}_{2} \mathrm{~N}_{14} \mathrm{O}_{5} \mathrm{~S}_{4}, 1127.1866$; found, 1127.1818.

(E)-2-((5-(7-Chloro-2-((3-((2-(3-)(5,5-difluoro-7,9-dimethyl-5H$5 \lambda^{4}, 6 \lambda^{4}$-dipyrrolo $\left[1,2-c: 2^{\prime}, 1^{\prime}-f\right][1,3,2]$ diazaborinin-3-yl)propanamido)ethyl)amino)-3-oxopropyl)amino)-4H-benzo[5,6]cyclohepta[1,2-d]thiazol-4-yl)-2-oxo-4-thioxo-3,4-dihydropyrimidin-1(2H)-yl)methyl)- $N$ - $(1 \mathrm{H}$-tetrazol-5-yl)thiazole-4-carboxamide (96). Following general procedure 3, 94 was converted to the BODIPYFL conjugate, 96 . This was purified by preparative RP-HPLC (Method 3), which isolated 96 at a retention time of $12.05 \mathrm{~min}$. This compound was freeze-dried to a red-green, iridescent solid $(0.21 \mathrm{mg}$, $0.22 \mu \mathrm{mol}, 11 \%)$. LC-MS (ESI+) Rt: $2.76 \min (254 \mathrm{~nm}$, Method 1); $(m / z): 971.4\left[\mathrm{M}\left({ }^{35} \mathrm{Cl}\right)+\mathrm{H}\right]^{+}$. H.MS-TOF $(\mathrm{ESI}+)(\mathrm{m} / z):[\mathrm{M}+\mathrm{H}]^{+}$ calcd for $\mathrm{C}_{41} \mathrm{H}_{39} \mathrm{BClF}_{2} \mathrm{~N}_{14} \mathrm{O}_{4} \mathrm{~S}_{3}, 971.2185$; found, 971.2189. $[\mathrm{M}+\mathrm{Na}]^{+}$ calcd for $\mathrm{C}_{41} \mathrm{H}_{38} \mathrm{BClF}_{2} \mathrm{~N}_{14} \mathrm{NaO}_{4} \mathrm{~S}_{3}, 993.2004$; found, 993.2002.

(E)-2-((5-)-Chloro-2-)((3-)(2-)(6-(2-)(4-(2-) (5,5-difluoro-7-(thiophen2-yl)-5H-4 $\lambda^{4}, 5 \lambda^{4}$-dipyrrolo[1,2-c:2' $\left.1^{\prime}-f\right][1,3,2]$ diazaborinin-3-yl)vinyl)phenoxy)acetamido)hexanamido)ethyl)amino)-3-oxopropyl)amino)-4H-benzo[5,6]cyclohepta[1,2-d]thiazol-4-yl)-2-oxo-4-thioxo-3,4-dihydropyrimidin-1(2H)-yl)methyl)- $\mathrm{N}$-(1H-tetrazol-5-yl)thiazole-4-carboxamide (97). Following general procedure 3, 94 was converted to the BODIPY630/650-X conjugate, 97. This was purified by preparative RP-HPLC (Method 4), which isolated 97 at a retention time of $11.28 \mathrm{~min}$. This compound was freeze-dried to a blue, iridescent solid (0.44 mg, $0.35 \mu \mathrm{mol}, 18 \%)$. LC-MS (ESI+) Rt: $2.99 \mathrm{~min}$ $\left(254 \mathrm{~nm}\right.$, Method 1); $(\mathrm{m} / z): 1242.4\left[\mathrm{M}\left({ }^{35} \mathrm{Cl}\right)+\mathrm{H}\right]^{+}$. H.MS-TOF (ESI+) $(\mathrm{m} / z):[\mathrm{M}+\mathrm{H}]^{+}$calcd for $\mathrm{C}_{56} \mathrm{H}_{52} \mathrm{BClF}_{2} \mathrm{~N}_{15} \mathrm{O}_{6} \mathrm{~S}_{4}, 1242.2852$; found, 1242.2858. $[\mathrm{M}+\mathrm{Na}]^{+}$calcd for $\mathrm{C}_{50} \mathrm{H}_{40} \mathrm{BClF}_{2} \mathrm{~N}_{14} \mathrm{NaO}_{5} \mathrm{~S}_{4}$, 1264.2671; found, 1264.2624.

(E)-2-((5-(7-Chloro-2-((3-)(2-(6-)(3-(5,5-difluoro-7,9-dimethyl-5H$5 \lambda^{4}, 6 \lambda^{4}$-dipyrrolo[1,2-c:2', $\left.1^{\prime}-f\right][1,3,2]$ diazaborinin-3-yl)propanamido)hexanamido)ethyl)amino)-3-oxopropyl)amino)- $4 \mathrm{H}$ benzo[5,6]cyclohepta[1,2-d]thiazol-4-yl)-2-oxo-4-thioxo-3,4-dihydropyrimidin-1(2H)-yl)methyl)- $\mathrm{N}-(1 \mathrm{H}$-tetrazol-5-yl)thiazole-4-carboxamide (98). Following general procedure 3, 94 was converted to the BODIPYFL-X conjugate, 98 . This was purified by preparative RP-HPLC (Method 4), which isolated 94 at a retention time of $12.69 \mathrm{~min}$. This compound was freeze-dried to a red-green, iridescent solid (0.35 mg, $0.31 \mu \mathrm{mol}, 18 \%)$. LC-MS (ESI+) Rt: $2.75 \mathrm{~min}(254$ nm, Method 1); $(m / z): 1084.6\left[\mathrm{M}\left({ }^{35} \mathrm{Cl}\right)+\mathrm{H}\right]^{+}$. H.MS-TOF (ESI+) $(\mathrm{m} / z):[\mathrm{M}+\mathrm{H}]^{+}$calcd for $\mathrm{C}_{47} \mathrm{H}_{50} \mathrm{BClF}_{2} \mathrm{~N}_{15} \mathrm{O}_{5} \mathrm{~S}_{3}, 1084.3026$; found, 
1085.3014. $[\mathrm{M}+\mathrm{Na}]+{ }^{+}$calcd for $\mathrm{C}_{47} \mathrm{H}_{49} \mathrm{BClF}_{2} \mathrm{~N}_{15} \mathrm{O}_{5} \mathrm{~S}_{3}, 1106.2845$; found, 1128.2825. H.MS-TOF (ESI-) $(\mathrm{m} / z)$ : $[\mathrm{M}-\mathrm{H}]^{-}$calcd for $\mathrm{C}_{47} \mathrm{H}_{48} \mathrm{BClF}_{2} \mathrm{~N}_{15} \mathrm{O}_{5} \mathrm{~S}_{3}, 1082.2880$; found, 1082.2839.

General Pharmacology Methods. cDNA Constructs. To create the NLuc-P2 $\mathrm{Y}_{2} \mathrm{R}$ constructs, human $\mathrm{P} 2 \mathrm{Y}_{2} \mathrm{R}$ DNA (obtained from the Missouri S\&T cDNA Resource Center) was amplified by PCR to remove the methionine start signal and cloned into PCR2.1 (linearized vector, Invitrogen). $\mathrm{P}_{2} \mathrm{Y}_{2} \mathrm{R}$ was then subcloned in-frame with the membrane-signal sequence of the $5 \mathrm{HT}_{3 \mathrm{~A}}$ receptor and the full-length sequence of nanoluciferase. The NLuc-tagged receptors expressed in $1321 \mathrm{~N} 1$ cells exhibited normal calcium signals $\left(\mathrm{EC}_{50}\right.$ for UTP $\gamma \mathrm{S}$ of $91 \pm 12 \mathrm{nM}, n=3)$.

Cell Culture and Cell-Line Generation. The $1321 \mathrm{~N} 1$ cells were maintained in Dulbecco's modified Eagle's medium (DMEM) containing $10 \%$ fetal calf serum (FCS HI) and $2 \mathrm{mM}$ L-glutamine at $37{ }^{\circ} \mathrm{C}$ with $5 \% \mathrm{CO}_{2}$. Mixed-population NLuc-P2Y2R-1321N1 cell lines were generated by transfecting the NLuc- $\mathrm{P}_{2} \mathrm{Y}_{2} \mathrm{R}$ construct using Lipofectamine 2000 (Life Technologies) according to the manufacturer's instructions and then subjecting the cells to selective pressure $(1 \mathrm{mg} / \mathrm{mL} \mathrm{G418)}$ for $2-3$ weeks. The mixed cell population was then dilution-cloned to obtain cell lines originating from a single cell. Screening for the active clones was initially performed with the calcium-mobilization assay and was followed by the detection of luminescence upon the addition of the NLuc substrate, furimazine. On this basis, a single, active, cloned cell line was selected for use in all of the NanoBRET assays. The $1321 \mathrm{~N} 1$ cells expressing wild-type $\mathrm{P}_{2} \mathrm{Y}_{2} \mathrm{R}\left(\mathrm{P} 2 \mathrm{Y}_{2} \mathrm{R}-1321 \mathrm{~N} 1\right)$ were gifted by Dr. Elizabeth Rosethorne, University of Nottingham.

Calcium-Mobilization Assay. $\mathrm{P}_{2} \mathrm{Y}_{2}-1321 \mathrm{~N} 1$ cells seeded into black-sided 96-well view plates were incubated at $37^{\circ} \mathrm{C}$ without $\mathrm{CO}_{2}$ for $45 \mathrm{~min}$ in a total volume of $100 \mu \mathrm{L}$ of a HEPES-buffered saline solution (HBSS; $10 \mathrm{mM}$ HEPES, $10 \mathrm{mM}$ glucose, $145 \mathrm{mM} \mathrm{NaCl}$, $5 \mathrm{mM} \mathrm{KCl}, 1 \mathrm{mM} \mathrm{MgSO}, 2 \mathrm{mM}$ sodium pyruvate, and $1.3 \mathrm{mM}$ $\mathrm{CaCl}_{2}$ ) containing $2.5 \mathrm{mM}$ probenecid, $2.3 \mu \mathrm{M}$ Fluo $4 \mathrm{AM}, 0.023 \%$ pluronic acid, $0.5 \mathrm{mM}$ Brilliant Black, $1 \mathrm{U} / \mathrm{mL}$ apyrase, and the ligand under investigation or the vehicle. The plates were then loaded onto a plate reader (FLEXstation, Molecular Devices), and the fluorescence was measured (excitation: $485 \mathrm{~nm}$, emission: $525 \mathrm{~nm}$ ) every $1.52 \mathrm{~s}$ for up to $200 \mathrm{~s}$ after the addition of UTP $\gamma \mathrm{S}$ at $15 \mathrm{~s}$. For the investigation of the BODIPYFL-labeled ligands, Fluo 4AM was replaced with $2.0 \mu \mathrm{M}$ X-Rhod-1 AM (excitation: $584 \mathrm{~nm}$, emission: $612 \mathrm{~nm}$ ).

NanoBRET Assay. The saturation and competition binding assays were performed according to the methodology of Stoddart. ${ }^{18}$ Briefly, the assays were performed on stably transfected $\mathrm{NLucP}_{2} \mathrm{Y}_{2}-1321 \mathrm{~N} 1$ cells that had been seeded $24 \mathrm{~h}$ prior to the experiment in white Thermo Scientific Matrix 96-well microplates. The medium in each well was removed and replaced with HBSS containing apyrase $(1 \mathrm{U} / \mathrm{mL})$ and the required concentration of the fluorescent ligand with or without the competing ligand. Upon the addition of the fluorescent ligand, the cells were incubated for $1 \mathrm{~h}$ at $37{ }^{\circ} \mathrm{C}$ without $\mathrm{CO}_{2}$. The NLuc substrate, furimazine (Promega), was then added to a final concentration of $10 \mu \mathrm{M}$, and the plate was incubated for a further $5 \mathrm{~min}$ at $37^{\circ} \mathrm{C}$ without $\mathrm{CO}_{2}$. The luminescence and resulting BRET were measured using a PHERAstar FS plate reader (BMG Labtech) at room temperature. For the assays involving 97 , sequential measurements of the filtered light emissions were made at $460 \mathrm{~nm}$ ( $80 \mathrm{~nm}$ bandpass) and $>610 \mathrm{~nm}$ (long-pass), and the raw BRET ratios were calculated by dividing the $>610 \mathrm{~nm}$ emissions by the $460 \mathrm{~nm}$ emissions. For the assays involving 98, the measurements were made at $475 \mathrm{~nm}$ ( $30 \mathrm{~nm}$ bandpass) and $535 \mathrm{~nm}$ ( $30 \mathrm{~nm}$ bandpass), and the raw BRET ratios were calculated by dividing the $535 \mathrm{~nm}$ emissions by the $475 \mathrm{~nm}$ emissions.

Confocal Microscopy. The $\mathrm{P}_{2} \mathrm{Y}_{2} \mathrm{R}-1321 \mathrm{~N} 1$ cells were grown to approximately $80 \%$ confluency on eight-well Labtek chambered cover glasses (Nunc Nalgene) in normal growth medium. The growth medium was removed and replaced with HBSS containing apyrase $(1 \mathrm{U} / \mathrm{mL})$ and either $1(10 \mu \mathrm{M})$ or the vehicle, and the cells incubated for $30 \mathrm{~min}$ at $37^{\circ} \mathrm{C}$ without $\mathrm{CO}_{2}$. The cells were then incubated with 97 or 98 at the required concentration for $10 \mathrm{~min}$ at room temperature prior to the collection of the single-equatorial confocal images. The images were obtained on a Zeiss LSM710 confocal microscope using a 40× c-Apochromat 1.2NA water-immersion objective. For 97, the images were collected using a $633 \mathrm{~nm}$ excitation wavelength and a 488/561/633 dichroic, and the emissions were collected through a 650LP filter. For 98, a $488 \mathrm{~nm}$ excitation wavelength was used with the same dichroic, and the emissions were collected using an LP575 filter. In each case, a pinhole diameter of 1 airy unit was used, and the laser power, gain, and offset were kept the same for all of the samples within each experiment. For both $\mathbf{9 7}$ and $\mathbf{9 8}$, the images presented are as representative of an individual experiment with matched conditions. Linear adjustments to the image brightness and contrast have been applied equally across all of the comparative images using Zen software in order to prepare the images for presentation.

Data Analysis. All of the data were analyzed using GraphPad Prism 6.

For the calcium-mobilization experiments, as none of the compounds synthesized as part of this study showed any partialagonist actions, the estimated affinity values $\left(\mathrm{p} K_{\mathrm{b}}\right)$ were calculated from the shifts of the agonist-concentration-response curves in the presence of the fluorescent antagonists using eq 1 :

$$
\mathrm{DR}=1+\frac{[\mathrm{b}]}{K_{\mathrm{b}}}
$$

where DR (dose ratio) is the ratio of the agonist concentration required to stimulate an identical response in the presence and absence of the antagonist, $\mathrm{b}$. The $\mathrm{p} K_{\mathrm{b}}$ is $-\log K_{\mathrm{b}}$.

The total and nonspecific saturation binding curves were fitted simultaneously using eq 2 :

$$
\text { BRET ratio }=\frac{B_{\max } \times[\mathrm{b}]}{[\mathrm{b}]+K_{\mathrm{d}}}+((M \times[\mathrm{b}])+C)
$$

where $B_{\max }$ is the maximum specific BRET signal, $[\mathrm{b}]$ is the nanomolar concentration of the fluorescent ligand, $K_{\mathrm{d}}$ is the equilibrium dissociation constant in nanomolar, $M$ is the slope of the nonspecificbinding component, and $C$ is the intercept with the $Y$-axis. The $\mathrm{p} K_{\mathrm{d}}$ is $-\log \mathrm{K}_{\mathrm{d}}$.

The competition binding curves were fitted using eq 3 :

$$
K_{\mathrm{i}}=\frac{\mathrm{IC}_{50}}{1+\frac{[\mathrm{L}]}{K_{\mathrm{d}}}}
$$

where [L] is the nanomolar concentration of 98 , and $K_{d}$ is the equilibrium dissociation constant of $\mathbf{9 8}$ in nanomolar. The $\mathrm{IC}_{50}$ was calculated as in eq 4 :

$$
\% \text { inhibition of specific binding }=\frac{100 \times[\mathrm{A}]}{[\mathrm{A}]+\mathrm{IC}_{50}}
$$

where $[\mathrm{A}]$ is the concentration of the unlabeled competing drug, and the $\mathrm{IC}_{50}$ is the molar concentration of the competing ligand required to inhibit $50 \%$ of the specific binding of the labeled ligand at a given concentration $([\mathrm{L}])$.

\section{ASSOCIATED CONTENT}

\section{S Supporting Information}

The Supporting Information is available free of charge on the ACS Publications website at DOI: 10.1021/acs.jmedchem.8b00139.

Molecular-formula strings (CSV)

Description of the VCD structure determination and figure showing the binding of the BODIPY630/650labeled (78-84) and BODIPYFL-labeled (85-91) ligands in NLuc-P2 $\mathrm{Y}_{2}-1321 \mathrm{~N} 1$ cells using the NanoBRET assay (PDF)

\section{AUTHOR INFORMATION}

\section{Corresponding Author}

*E-mail: michael.stocks@nottingham.ac.uk. Tel.: +44 (0)115 9515151. 


\section{ORCID}

Barrie Kellam: 0000-0003-0030-9908

Michael J. Stocks: 0000-0003-3046-137X

\section{Author Contributions}

${ }^{\perp}$ S.C. and N.D.K. contributed equally to this work. S.J.H., B.K., and M.J.S. conceived the study and managed the project. S.C., N.D.K., and M.J.S. performed the chemical syntheses. R.J.L. performed the VCD structural determination. S.J.H., L.A.S., S.C., N.D.K., B.K., and M.J.S. participated in the research design. J.G. conducted the pharmacology experiments. J.G., L.A.S., and S.J.H. performed the pharmacology-data analysis. All of the authors wrote or contributed to the writing of the manuscript.

\section{Funding}

This work was supported by the U.K. Medical Research Council (grant numbers MR/L016389/1 and MR/N020081/1) and an MRC-funded research studentship for S.C. (MRC-funded Ph.D. studentship reference number 1365529).

\section{Notes}

The authors declare no competing financial interest.

\section{ACKNOWLEDGMENTS}

The authors thank Dr. Elizabeth Rosethorne for supplying the $\mathrm{P}_{2} \mathrm{Y}_{2}-1321 \mathrm{~N} 1$ astrocytoma cells, Mr. Nickolaj Groenewoud for assistance with the molecular biology, and Miss Seema Rajani for assistance with the confocal-microscopy work.

\section{ABBREVIATIONS USED}

ATP,adenosine 5'-triphosphate; BODIPY,boron-dipyrromethene; BODIPY,boron-dipyrromethene; BRET,bioluminescence-resonance-energy transfer; ESI,electrospray ionization; FC,flash chromatography; GPCR,G-protein-coupled receptor; HPLC,high-performance liquid chromatography; HRMS,highresolution mass spectrometry; $K_{d}$ dissociation constant of a labeled ligand-receptor complex; $K_{\mathrm{i}}$ dissociation constant of a ligand-receptor complex determined through a binding assay; LC-MS,liquid chromatography-mass spectrometry; MW, microwave; NanoBRET,nanoluciferase-bioluminescence-resonance-energy transfer; NLuc,nanoluciferase; PREP,preparative; RM,reaction mixture; RP,reverse phase; SAR,structure-activity relationship; TLC,thin-layer chromatography; UTP $\gamma$ S, uridine$5^{\prime}$-( $\gamma$-thio)-triphosphate; VCD,vibrational circular dichroism

\section{REFERENCES}

(1) Abbracchio, M. P. International Union of Pharmacology LVIII: Update on the P2Y G Protein-Coupled Nucleotide Receptors: From Molecular Mechanisms and Pathophysiology to Therapy. Pharmacol. Rev. 2006, 58 (3), 281-341.

(2) Burnstock, G.; Kennedy, C. Is There a Basis for Distinguishing Two Types of P2-Purinoceptor? Gen. Pharmacol. 1985, 16 (5), 433440.

(3) Lazarowski, E. R.; Watt, W. C.; Stutts, M. J.; Boucher, R. C.; Harden, T. K. Pharmacological Selectivity of the Cloned Human P2UPurinoceptor: Potent Activation by Diadenosine Tetraphosphate. Br. J. Pharmacol. 1995, 116 (1), 1619-1627.

(4) Conroy, S.; Kindon, N.; Kellam, B.; Stocks, M. J. Drug-like Antagonists of P2Y Receptors-From Lead Identification to Drug Development. J. Med. Chem. 2016, 59 (22), 9981-10005.

(5) Schumacher, D.; Strilic, B.; Sivaraj, K. K.; Wettschureck, N.; Offermanns, S. Platelet-Derived Nucleotides Promote Tumor-Cell Transendothelial Migration and Metastasis via $\mathrm{P}_{2} \mathrm{Y}_{2}$ Receptor. Cancer Cell 2013, 24 (1), 130-137.
(6) Di Virgilio, F.; Falzoni, S.; Giuliani, A. L.; Adinolfi, E. P2 Receptors in Cancer Progression and Metastatic Spreading. Curr. Opin. Pharmacol. 2016, 29, 17-25.

(7) Müller, T.; Fay, S.; Vieira, R. P.; Karmouty-Quintana, H.; Cicko, S.; Ayata, K.; Zissel, G.; Goldmann, T.; Lungarella, G.; Ferrari, D.; Di Virgilio, F.; Robaye, B.; Boeynaems, J.-M.; Blackburn, M. R.; Idzko, M. The Purinergic Receptor Subtype $\mathrm{P}_{2} \mathrm{Y}_{2}$ Mediates Chemotaxis of Neutrophils and Fibroblasts in Fibrotic Lung Disease. Oncotarget 2017, 8 (22), 35962-35972.

(8) Kindon, N.; Davis, A.; Dougall, I.; Dixon, J.; Johnson, T.; Walters, I.; Thom, S.; McKechnie, K.; Meghani, P.; Stocks, M. J. From UTP to AR-C118925, the Discovery of a Potent Non Nucleotide Antagonist of the $\mathrm{P}_{2} \mathrm{Y}_{2}$ Receptor. Bioorg. Med. Chem. Lett. 2017, 27 (21), 48494853.

(9) Kindon, N. D.; Meghani, P.; Thom, S. Preparation of 2-oxo-4thioxopyrimidin-1-ylmethylheterocyclylcarboxylates as P2-purinoceptor 7-transmembrane G-protein Coupled Receptor Antagonists. WO9854180, 1998.

(10) Kemp, P. A.; Sugar, R. A.; Jackson, A. D. Nucleotide-Mediated Mucin Secretion from Differentiated Human Bronchial Epithelial Cells. Am. J. Respir. Cell Mol. Biol. 2004, 31 (4), 446-455.

(11) Rafehi, M.; Burbiel, J. C.; Attah, I. Y.; Abdelrahman, A.; Müller, C. E. Synthesis, Characterization, and in Vitro Evaluation of the Selective $\mathrm{P}_{2} \mathrm{Y}_{2}$ Receptor Antagonist AR-C118925. Purinergic Signalling 2017, 13 (1), 89-103.

(12) Jacobson, K. A.; Ivanov, A. A.; de Castro, S.; Harden, T. K.; Ko, H. Development of Selective Agonists and Antagonists of P2Y Receptors. Purinergic Signalling 2009, 5 (1), 75-89.

(13) Jayasekara, P. S.; Barrett, M. O.; Ball, C. B.; Brown, K. A.; Hammes, E.; Balasubramanian, R.; Harden, T. K.; Jacobson, K. A. 4Alkyloxyimino Derivatives of Uridine-5'-Triphosphate: Distal Modification of Potent Agonists as a Strategy for Molecular Probes of $\mathrm{P}_{2} \mathrm{Y}_{2}$

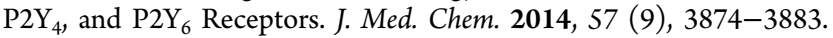

(14) Böhme, I.; Beck-Sickinger, A. G. Illuminating the Life of GPCRs. Cell Commun. Signaling 2009, 7 (1), 16.

(15) Ma, Z.; Du, L.; Li, M. Toward Fluorescent Probes for GProtein-Coupled Receptors (GPCRs). J. Med. Chem. 2014, 57 (20), 8187-8203.

(16) Sridharan, R.; Zuber, J.; Connelly, S. M.; Mathew, E.; Dumont, M. E. Fluorescent Approaches for Understanding Interactions of Ligands with G Protein Coupled Receptors. Biochim. Biophys. Acta, Biomembr. 2014, 1838 (1), 15-33.

(17) Cottet, M.; Faklaris, O.; Zwier, J. M.; Trinquet, E.; Pin, J.-P.; Durroux, T. Original Fluorescent Ligand-Based Assays Open New Perspectives in G-Protein Coupled Receptor Drug Screening. Pharmaceuticals 2011, 4 (12), 202-214.

(18) Stoddart, L. A.; Johnstone, E. K. M.; Wheal, A. J.; Goulding, J.; Robers, M. B.; Machleidt, T.; Wood, K. V.; Hill, S. J.; Pfleger, K. D. G. Application of BRET to Monitor Ligand Binding to GPCRs. Nat. Methods 2015, 12 (7), 661-663.

(19) Hansen, A. H.; Sergeev, E.; Pandey, S. K.; Hudson, B. D.; Christiansen, E.; Milligan, G.; Ulven, T. Development and Characterization of a Fluorescent Tracer for the Free Fatty Acid Receptor 2 (FFA2/GPR43). J. Med. Chem. 2017, 60 (13), 5638-5645.

(20) Instant JChem, 16.2.15.0 2016; ChemAxon: Budapest, 2016.

(21) Kindon, N. D.; Meghani, P.; Thom, S. Preparation of Pyrimidinediones and Thioxopyrimidinones as P2-purinoceptor 7transmembrane (TM) G-protein Coupled Receptor Antagonists. WO9926944, 1999.

(22) He, Y.; Bo, W.; Dukor, R. K.; Nafie, L. A. Determination of Absolute Configuration of Chiral Molecules Using Vibrational Optical Activity: A Review. Appl. Spectrosc. 2011, 65 (7), 699-723.

(23) Yaguchi, Y.; Nakahashi, A.; Miura, N.; Taniguchi, T.; Sugimoto, D.; Emura, M.; Zaizen, K.; Kusano, Y.; Monde, K. Vibrational CD (VCD) Spectroscopy as a Powerful Tool for Chiral Analysis of Flavor Compounds. ACS Symp. Ser. 2015, 1212, 35-56.

(24) Izumi, H.; Ogata, A.; Nafie, L. A.; Dukor, R. K. Structural Determination of Molecular Stereochemistry Using VCD Spectroscopy and a Conformational Code: Absolute Configuration and Solution 
Conformation of a Chiral Liquid Pesticide, (R)-(+)-Malathion. Chirality 2009, 21 (1E), E172-E180.

(25) Patel, Y.; Gillet, V. J.; Howe, T.; Pastor, J.; Oyarzabal, J.; Willett, P. Assessment of Additive/Nonadditive Effects in Structure-Activity Relationships: Implications for Iterative Drug Design. J. Med. Chem. 2008, 51 (23), 7552-7562.

(26) Khatuya, H.; Hutchings, R. H.; Kuo, G.-H.; Pulito, V. L.; Jolliffe, L. K.; Li, X.; Murray, W. V. Arylpiperazine Substituted Heterocycles as Selective $\alpha$ la Adrenergic Antagonists. Bioorg. Med. Chem. Lett. 2002, 12 (17), 2443-2446.

(27) Machleidt, T.; Woodroofe, C. C.; Schwinn, M. K.; Méndez, J.; Robers, M. B.; Zimmerman, K.; Otto, P.; Daniels, D. L.; Kirkland, T. A.; Wood, K. V. NanoBRET - A Novel BRET Platform for the Analysis of Protein-Protein Interactions. ACS Chem. Biol. 2015, 10 (8), $1797-1804$.

(28) Christiansen, E.; Hudson, B. D.; Hansen, A. H.; Milligan, G.; Ulven, T. Development and Characterization of a Potent Free Fatty Acid Receptor 1 (FFA1) Fluorescent Tracer. J. Med. Chem. 2016, 59 (10), 4849-4858.

(29) Lohse, M. J.; Nuber, S.; Hoffmann, C. Fluorescence/ Bioluminescence Resonance Energy Transfer Techniques to Study G-Protein-Coupled Receptor Activation and Signaling. Pharmacol. Rev. 2012, 64 (2), 299-336.

(30) Vernall, A. J.; Stoddart, L. A.; Briddon, S. J.; Ng, H. W.; Laughton, C. A.; Doughty, S. W.; Hill, S. J.; Kellam, B. Conversion of a Non-Selective Adenosine Receptor Antagonist into A3-Selective High Affinity Fluorescent Probes Using Peptide-Based Linkers. Org. Biomol. Chem. 2013, 11 (34), 5673.

(31) 2,4-Dithi(oxo)-pyrimidin-5-yl compounds bearing a tricyclic substituent useful as P2 purinoceptor antagonists. US6107297, Aug 22, 2000. 